УДК 004.415.2, 004.588

\title{
HUMAN BEING EMOTION IN COGNITIVE INTELLIGENT ROBOTIC CONTROL. PT. I: QUANTUM / SOFT COMPUTING APPROACH ${ }^{1}$
}

\author{
Mamaeva Alla1, Shevchenko Andrey ${ }^{2}$, Ulyanov Sergey ${ }^{3}$, Feng Maria ${ }^{4}$, Yamafuji Kazuo ${ }^{5}$
}

${ }^{1}$ PhD Student;

Dubna State University,

Institute of system analysis and management;

141980, Dubna, Moscow reg., Universitetskaya str., 19;

e-mail: allamamaeva.d@gmail.com.

${ }^{2}$ PhD Student;

Dubna State University,

Institute of system analysis and management;

141980, Dubna, Moscow reg., Universitetskaya str., 19;

e-mail: ashevchenko.uni@gmail.com.

${ }^{3}$ Doctor of Science in Physics and Mathematics, professor;

Dubna State University,

Institute of system analysis and management;

141980, Dubna, Moscow reg., Universitetskaya str., 19;

INESYS (EFKO);

e-mail:ulyanovsv@mail.ru.

${ }^{4} \mathrm{PhD}$;

Civil Engineering and Engineering Mechanics,

Columbia University;

500 W 120th Street, 610 Mudd, New York, NY 10027, USA;

e-mail:mfeng@columbia.edu.

${ }^{5} \mathrm{PhD}$, professor;

Dept. of Mechanical and Control Eng.,

University of Electro-Communications;

1-5-1 Chofu, Chofugaoka,182 Tokyo, Japan;

e-mail: yamafuji@yama.mce.uec.ac.jp.

The article consists of two parts. Part I shows the possibility of quantum / soft computing optimizers of knowledge bases (QSCOptKB ${ }^{\mathrm{TM}}$ ) as the toolkit of quantum deep machine learning technology implementation in the solution's search of intelligent cognitive control tasks applied the cognitive helmet as neurointerface. In particular case, the aim of this part is to demonstrate the possibility of classifying the mental states in on line of a human being operator with knowledge extraction from electroencephalograms based on SCOptKB ${ }^{\mathrm{TM}}$ and QCOptKB ${ }^{\mathrm{TM}}$ sophisticated toolkit. Application of soft computing technologies to identify objective indicators of the psychophysiological state of an examined person described. The role and necessity of applying intelligent information technologies development based on computational intelligence toolkits in the task of objective estimation of a general psychophysical state of a human being operator shown. Developed information technology examined with special (difficult in diagnostic practice) examples emotion state estimation of autism children (ASD) and dementia and background of the knowledge bases design for intelligent robot of service use is it. Application of cognitive intelligent control in navigation of autonomous robot for avoidance of obstacles demonstrated.

Keywords: neural interface, computational intelligence toolkit, intelligent control system, deep machine learning, emotions, quantum soft computing optimizer.

\footnotetext{
${ }^{1}$ The development implemented as part of UNESCO International project "Robotic Non / Industrial Intelligent Cognitive Control Systems in Hazard Situations: Toolkit of end-to-end quantum soft computational intelligence IT" [with the participation and accomplices: Columbia University and Princeton University, USA; Università degli Studi di Milano (Crema), Italy; University of ElectroCommunications, (Tokyo, Chofu) Japan; Nagoya University, Japan; State University "Dubna, RF] with support from ST Microelectronics, Italy; Yamaha Motor Co., (Iwata) Japan and EFKO Group of Companies (“INESIS” LLC), RF.
} 


\title{
ЭМОЦИИ ЧЕЛОВЕКА В КОГНИТИВНОМ ИНТЕЛЛЕКТУАЛЬНОМ РОБОТИЗИРОВАННОМ УПРАВЛЕНИИ. Ч. 1: ПОДХОД НА КВАНТОВЫХ / МЯГКИХ ВЫЧИСЛЕНИЯХ
}

\author{
Мамаева Алла Александровна ${ }^{1}$, Шевченко Андрей Владимирович ${ }^{2}$, \\ Ульянов Сергей Викторович ${ }^{3}$, Фэн Мария ${ }^{4}$, Ямафуджи Кацуо $^{5}$
}

\author{
${ }^{I}$ Аспирант; \\ ГБОУ ВО МО «Университет «Дубна», \\ Институт системного анализа и управления; \\ 141980, Московская обл., г. Дубна, ул. Университетская, 19; \\ e-mail: allamamaeva.d@gmail.com. \\ ${ }^{2}$ Аспирант; \\ ГБОУ ВО МО «Университет «Дубна», \\ Институт системного анализа и управления; \\ 141980, Московская обл., г. Дубна, ул. Университетская, 19; \\ e-mail: ashevchenko.uni@gmail.com. \\ ${ }^{3}$ Доктор физико-математических наук, профессор; \\ ГБОУ ВО МО «Университет «Дубна», \\ Институт системного анализа и управления; \\ 141980, Московская обл., г. Дубна, ул. Университетская, 19; \\ ИНЭСИС (ЭФКО); \\ e-mail: ulyanovsv@mail.ru.

\section{${ }^{4}$ Доктор наук;} \\ Промышленное и гражданское строительств, \\ Колумбийский универсистет, \\ 500 W 120th Street, 610 Mudd, Нью-Йорк, NY 10027, США; \\ e-mail: mfeng@columbia.edu. \\ ${ }^{5}$ Доктор наук, профессор, \\ Факультет механики и технической кибернетики (интеллектуальные системы), \\ Университет передачи информации; \\ 1-5-1, Япония, Токио, Chofu, Chofugaoka ,182; \\ e-mail: yamafuji@yama.mce.uec.ac.jp.
}

Статья состоит из двух частей. Часть 1 показывает возможность реализации технологии квантового глубокого машинного обучения на основе квантового оптимизатора баз знаний на мягких вычислениях в задачах когнитивного интеллектуального управления с использованием когнитивного илема в качестве нейроинтерфейса. Целью этой части статьи является демонстрация возможности классификации ментальных состояний человека-оператора с извлечением знаний из электроэнцефалограмм на основе инструментариев SCOptKB ${ }^{\mathrm{TM}}$ и QCOptKB ${ }^{\mathrm{TM}}$. Описано применение технологий мягких вычислений для выявления объективных показателей психофизиологического состояния исследуемого человека. Показана роль и необходимость применения интеллектуальных информационных технологий на основе интеллектуального инструментария в задаче объективной оценки общего психофизического состояния человека-оператора. Разработанная информационная технология рассмотрена на особых (сложных в диагностической практике) примерах оценки эмоционального состояния детей, страдающих аутизмом, а также описан процесс создания базы знаний для интеллектуального робота сервисного обслужсивания. Показано применение интеллектуального когнитивного управления в навигачии автономного робота для обхода препятствий.

Ключевые слова: нейронная сеть, интеллектуальная система управления, глубокое машинное обучение, эмоции, квантовый оптимизатор баз знаний, мягкие вычисления. 


\section{Introduction}

The state-of-the-art sensing and processing tools, health-monitoring technologies attract significant attention in research and industry in the last three decades [1,2]. The inclusion of human being operator in the feedback loop of intelligent control systems (ICS) for decision-making in complex situations creates both an information resource that can improve the efficiency of the development and application of ICS. Unfortunately, it is often associated with an increasing in the information risk of hazard situations due to the presence of an unpredictable human health-monitoring and emotion state factors [2].

Thus, it is necessary to have quantitative and qualitative indicators that would not depend on the individual characteristics of the human being emotion operator and at the same time guaranteed objectivity of the obtained indicators. In that case, the developed ICS will be able to perceive, adapt and make decisions in difficult situations [3] due to the inclusion in the structure of these indicators as criteria for the quality of intelligent control.

\section{Cognitive intelligent control problems}

\subsection{Tasks of hybrid cognitive and intelligent control}

A number of studies [4-7] showed the possibility of development a simplified mathematical model of emotions. But due to physical limitations, the trade-off of informational boundaries on the applicability of the developed model also have a significant influence on the correctness of description and reliability of the extracted knowledge from the imperfect mathematical model. In ICS theory, one of the effective approaches to the risk decreasing of decision-making is the development of robust ICS structures with corresponding knowledge bases (KBs).

The problems of physical limitations and information boundaries solved by the possibility of forming KB with the required level of robustness in the design process of ICS by extracting knowledge and valuable information from the dynamic behavior of the model of the physical control object [8].

Figure 1 demonstrate general structure of hybrid cognitive intelligent control system. The structure based on fuzzy and cognitive controllers, includes quantum fuzzy inference with quantum genetic algorithm in Box "Quantum computing KB optimizer" and are the background of quantum cognitive self-organized controller (see, in details Part II).

The main problem of cognitive intelligent control system (presented in Fig. 1) is design optimal robust control with minimal loss of value work and minimum of required initial information on external environments. 


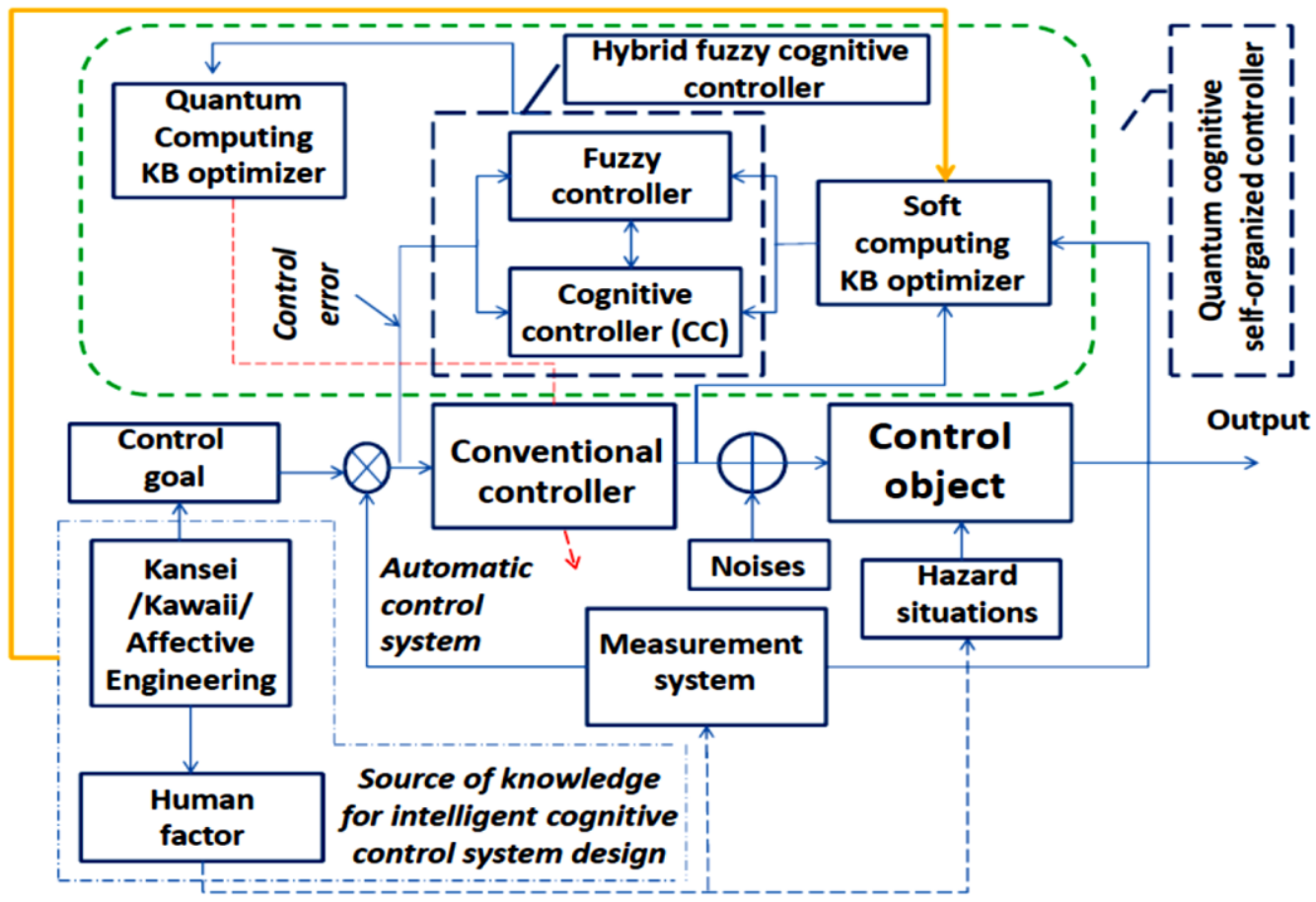

Figure 1. Structure of hybrid intelligent cognitive control system based on quantum soft computing

Let us consider briefly the solution of this problem using information-thermodynamic approach.

\subsection{Synergetic effect of information-thermodynamic trade-off interrelations between stability, controllability and robustness of robotic motion intelligent control}

Consider the distribution equation of the trade-off control qualities of a dynamic system $\dot{q}_{i}=\varphi(q, t, S(t), u)$ as control object in the form:

$$
\frac{d V}{d t}=\sum_{i=1}^{n} q_{i} \cdot \varphi(q, t, S(t), u)+\left(S_{p}-S_{c}\right) \cdot\left(\dot{S}_{p}-\dot{S}_{c}\right) \leq 0,
$$

where $S_{p}$ is an entropy production of control object (plant), $S_{c}$ is an entropy production of controller, $S=S_{p}-S_{c}$ is a generalized entropy production of dynamic control system.

Eq. (1) in analytical form relates such qualitative concepts of control theory as stability, controllability and robustness based on the concept of phenomenological thermodynamics entropy. Such an approach designs the necessary distribution between levels of stability, controllability and robustness, which allows achieving the control goal in emergencies with a minimum consumption of useful resource due to the application of the minimum generalized entropy production included in the right-hand side as a fitness function in the genetic algorithm.

Now let us looked at (1), taking into account the connection between thermodynamic entropy and Shannon's information entropy. The definitions of thermodynamic entropy $S$ and information entropy $H$ related by the von Neumann relation in the form:

$$
S=k H=-k \sum_{i} p_{i} \ln p_{i},
$$

where $k \approx 1.38 \cdot 10^{-23} \mathrm{~J} / K$ and is the Boltzmann constant.

In Eq. (1) replace $S(t)$ with the Shannon's entropy:

$$
\frac{d V}{d t}=\sum_{i=1}^{n} q_{i} \cdot \varphi\left(q, t,\left(H_{p}-H_{c}\right), u\right)+k\left(H_{p}-H_{c}\right) \cdot\left(\dot{H}_{p}-\dot{H}_{c}\right) \leq 0
$$


Thus, Eq. (3) also relates stability, controllability and robustness, but already based on Shannon's information entropy, which also allows one to determine control for guaranteed achievement of the control goal in emergencies with a minimum required amount of information about the external environment and the state of the control object.

A generalization of Eqs (1) and (3) is the following system of equations:

$$
\begin{aligned}
\frac{d V}{d t}=\sum_{i=1}^{n} q_{i} \cdot & \varphi\left(q, t,\left(S_{p}-\left(S_{\text {Int }}+S_{\text {Cog }}\right)\right), u\right)+\left(S_{p}-\left(S_{\text {Int }}+S_{\text {Cog }}\right)\right) \cdot\left(\dot{S}_{p}-\left(\dot{S}_{\text {Int }}+\dot{S}_{\text {Cog }}\right)\right) \\
\leq & 0 \\
\frac{d V}{d t}= & \sum_{i=1}^{n} q_{i} \cdot \varphi\left(q, t, k\left(H_{p}-\left(H_{\text {Int }}+H_{\text {Cog }}\right)\right), u\right)+k\left(H_{p}-\left(H_{\text {Int }}+H_{\text {Cog }}\right)\right) \\
& \cdot\left(\dot{H}_{p}-\left(\dot{H}_{\text {Int }}+\dot{H}_{\text {Cog }}\right)\right) \leq 0
\end{aligned}
$$

where $\left(S_{\text {Int }}+S_{\text {Cog }}\right)$ and $\left(H_{\text {Int }}+H_{\text {Cog }}\right)$ means total thermodynamic and information entropies of intelligent and cognitive controllers, respectively.

It follows from Eq. (4) that the robustness of an intelligent control system can increased by producing the minimum entropy (value information) of the cognitive controller, which reduces the loss of useful life (safety increasing), and Eq. (5) shows that the negentropy of the cognitive controller reduces the minimum requirements for the initial information to achieve robustness. Moreover, information action based on knowledge (in the knowledge base of the cognitive regulator) allows get an additional resource for useful work, which is equivalent to the appearance of a targeted action on the control object to ensure the guaranteed achievement of the control goal in uncertainty and information risk conditions.

Due to the synergetic effect, an additional information resource created and the multi-agent system is able to solve complex dynamic tasks for performing mutual work. The given task may not be fulfilled by each element (agent) of the system separately in various environments without external management, control or coordination, however, exchange of knowledge and information allows perform useful mutual work to achieve the management goal under the conditions of uncertainty of the initial information and limited consumption of useful resources. In particular, it known that for closed-loop control systems, the maximal amount of useful work $W$ that extracted with information amount satisfies the inequality:

$$
W_{\max }(t)=k \int_{0}^{t} T_{\min } \dot{I}_{c} d t^{\prime} \leq k T I
$$

where $k$ is the Boltzmann constant, $T_{\min }(t)$ is interpreted as the lowest achievable temperature by the system in time $t$ for feedback control, assuming $T_{\min }(0)=T$ and $I_{c}$ determines the amount of Shannon information (entropy transfer), extracted by the system from the measurement process $[9,10]$.

Figure 2 demonstrate logical interrelations of information role in process of work extraction and trade-off of control qualities. 


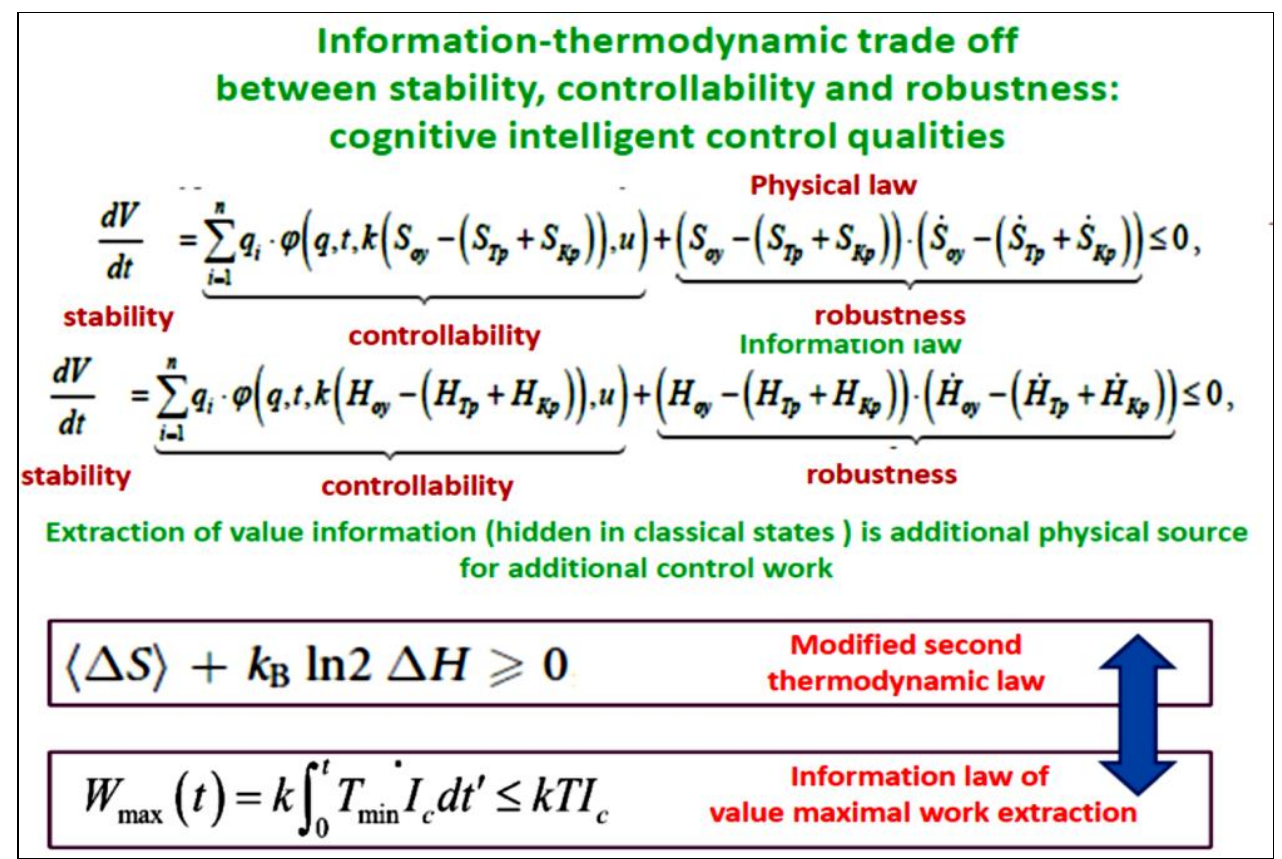

Figure 2. Interrelations between extracted work and information, and trade-off of control qualities

Physically, the synergetic effect means self-organization of knowledge and creation of additional information that allows the multi-agent system to perform the most useful work with a minimum loss of useful resource and with a minimum of the required initial information, without destroying the lower executive level of the control system [9]. Together with the information-thermodynamic law of intelligent control (optimal distribution of the management qualities "stability - controllability - robustness"), an intelligent control system (ICS) is designed with multi-agent systems, ensuring the achievement of the management goal under the conditions of uncertain initial information and limited useful resource [9-13].

\subsection{Extracted work and information}

If microscopic degrees of freedom are accessible to the observer in the form of the Maxwell demon, then the second law of thermodynamics may violate (see, Fig. 2). Szilard showed from an analysis of the Maxwell demon model that work is extracted from the thermodynamic cycle in the form as the amount, $k T \ln 2$. Moreover, in $[12,13]$ it shown that the recoverable work $W_{\text {ext }}^{S}$ from the system determined by the amount of information $I$ (or quantum-classical mutual information) that measures the knowledge of the system when measuring. At the same time, such a ratio in the form of a lower boundary exists for the total cost of measuring and erasing information $W_{\text {ext }}^{S} \leq-\Delta F^{S}+k T I$ and $W_{\text {ext }}^{M} \geq k T I$, where $\Delta F^{S}$ is determines the free energy of the system. Then it is easy to notice that the speed of the extracted work $\dot{W}_{\text {ext }}$ is limited by the value $\dot{W}_{\text {ext }} \leq$ $k T \dot{I}$, i.e., it is limited by the speed of the extracted information [13].

Let us consider a network of loosely coupled groups of robots working together to solve tasks that go beyond individual capabilities. Different nodes of such a system, as a rule, have a different intelligent level (knowledge, algorithms, and computational bases) and various information resources in designing. Each node should be able to modify its behavior depending on the circumstances, as well as to plan its communication and cooperation strategies with other nodes. Here the indicators of the level of cooperation are the nature of the distribution of tasks, the unification of various information resources and, of course, the possibility of solving a common problem in a given time.

\subsection{Quantum algorithm of knowledge self-organization}

A quantum algorithm (QA) model of ICS self-organization proposed in [9] based on the principles of minimum information entropy (in the "intelligent" state of control signals) and a generalized thermodynamic measure of entropy production (in the system "control object + controller"). The main result of the application of the self-organization process is the acquisition of the necessary level of robustness and the flexibility (adaptability) of the reproducible structure. It is noted that the property of robustness (by its physical nature) acts as an integral part of self-organization, and the required level of robustness of ICS is achieved by fulfilling the principle of minimum production of generalized entropy, which was noted above. 
The principle of minimum entropy production in control object and control system [14] serves as the physical principle of optimal functioning with a minimum consumption of useful work and underlies the development of robust ICS. This statement based on the fact that, for the general case of controlling dynamic objects, the optimal solution to the finite variation problem of determining the maximum of the useful work $W$ is equivalent, according to [15], to the solution of the finite variation problem of finding the minimum of the entropy production $S$. Therefore, the developed QA model used principle of minimum informational entropy guarantees the necessary condition for self-organization - the minimum of the required initial information in the teaching signals; the thermodynamic criterion of the minimum of a new measure of generalized entropy production provides a sufficient condition for self-organization - the robustness of control processes with a minimum consumption of useful resource.

More significant is the fact that the average amount of work done by dissipation force $\frac{\left\langle W_{\text {diss }}\right\rangle}{k T}=$ $S_{K L}\left(P_{F} \| P_{B}\right)$, i.e., the work of dissipation forces is determined by the Kullback-Leibler divergence for probability distributions $P_{F}, P_{B}$. Note that the left side of this relation represents physically thermal energy, and the right side defines purely information about the system. A similar relationship exists between the work produced by the forces of dissipation and the difference between generalized Renyi divergences [16].

Figure 3 illustrates the QA structure of self-organization (QASO) in design process of robust intelligent PID-controller with application of quantum fuzzy inference with quantum genetic algorithm for choice the optimal quantum correlation type between PID-controller coefficient gains in temporal schedule.

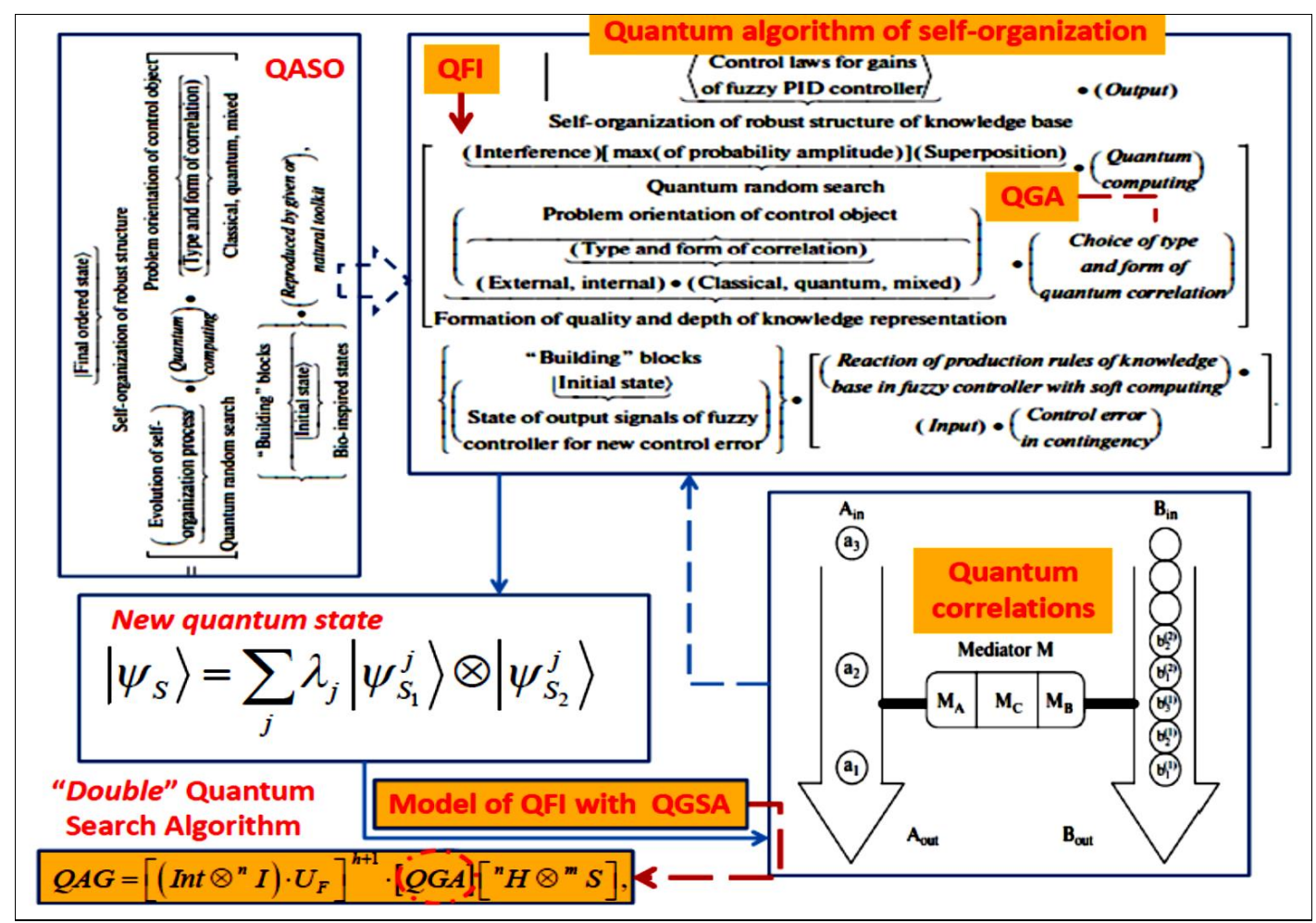

Figure 3. Quantum algorithm of self-organization based on quantum fuzzy inference and quantum genetic algorithm

Thus, substituting the relations between the information and the extracted free energy and work in (4) and (5), we obtain the conclusion (noted above) that the robustness of the intelligent control system can increased by producing the entropy of the cognitive controller. The optimal cognitive controller reduces the loss of useful resource of the control object, and negentropy of the cognitive regulator reduces the requirements for minimum initial information to achieve robustness. Therefore, the extracted information, based on knowledge (in the knowledge base of the cognitive controller), allows to get an additional resource for useful work, which is equivalent to the appearance of a targeted action on the control object to guarantee the achievement of the control goal in unpredicted situations.

Let us consider briefly Brain Emotional Learning Based Intelligent Controller (BELBIC) structure [17] as the consequence of the intelligent cognitive control system on Fig. 3. 
Example. In a biological system, emotional responses of human being operator are utilized for fast decision-making in complex environments or emergencies. It is thought that the amygdala and the orbitofrontal cortex are the most important parts of the brain involved in emotional responses and learning. The amygdala is a small structure in the medial temporal lobe of the brain that is thought to be responsible for the emotional evaluation of stimuli (see, Appendices 1 and 2). This evaluation is in turn used as a quantum basis for emotional states and responses and is used for attention signals and laying down long-term memories. The amygdala and the orbitofrontal cortex compute their outputs based on the emotional signal (the reinforcing signal) received from the environment. The final output (the emotional responses) calculated by subtracting the amygdala's output from the orbitofrontal cortex's (OFC) output (see Fig. 4).

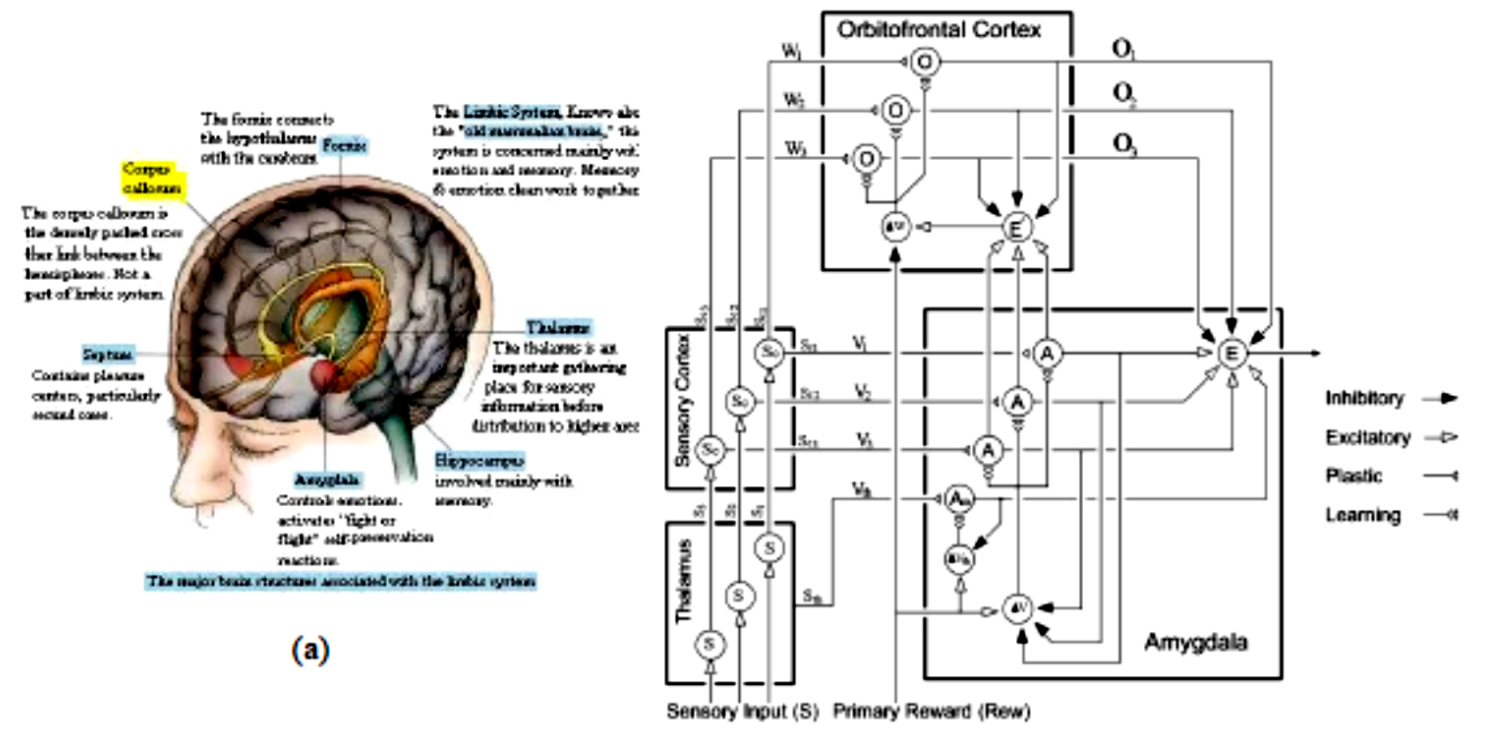

Figure 4. a) Sectional view of the human brain for emotion processing. b) Graphical depiction of the developed computational model of brain [18]

It should observe that it essentially converts two sets of inputs (sensory inputs and emotional cues or reinforcing signals) into the decision signal (the emotional responses) as its output. Closed loop configurations using this block (BELBIC) in the feed-forward-loop of the total system in an appropriate manner have implemented so that the input signals have the proper interpretations. The block implicitly implemented the critic, the learning algorithm and the action selection mechanism used in the functional implementations of emotionally-based (or, generally, reinforcement learning-based) controllers, all at the same time.

The policies design for PID-based controller and the BELBIC controller are the same due to the equal number of states, which needed for the feedback. The structure of the control circuit using the direct-adaptive control strategy illustrated in Fig. 5.

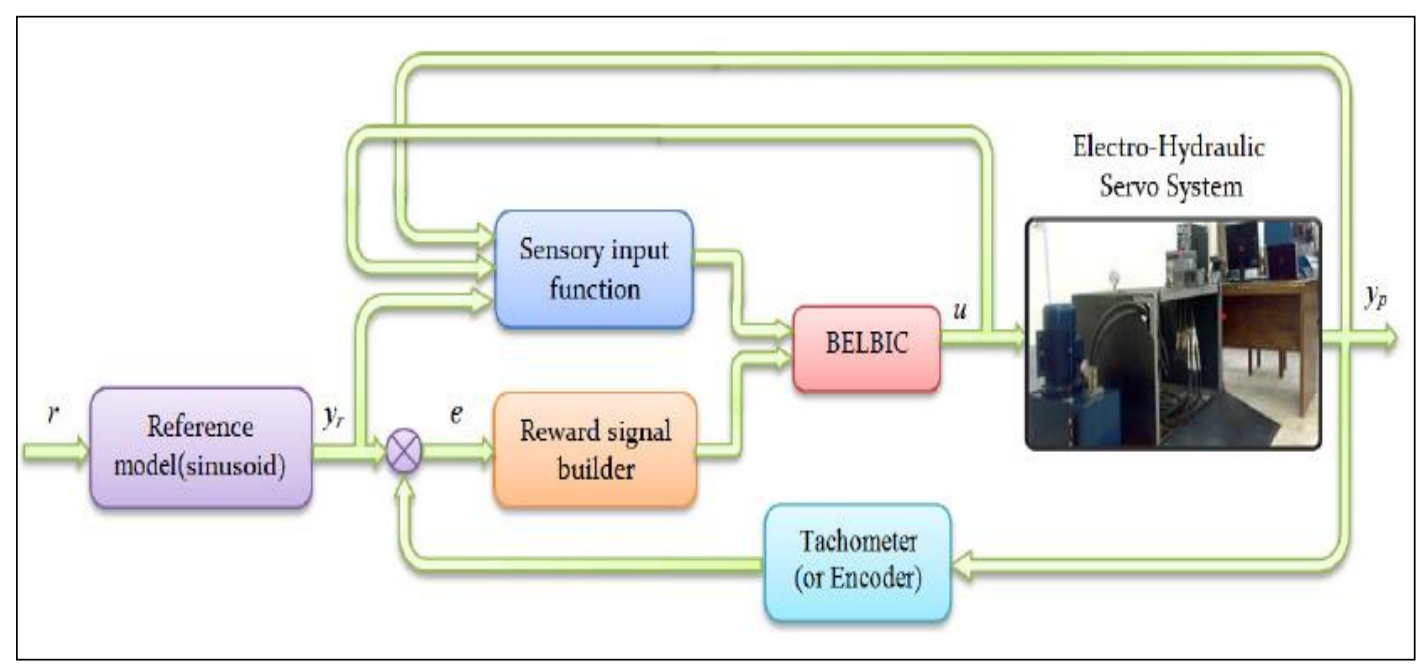

Figure 5. System configuration using brain emotional controller 
The PID controller contains a constant steady-state position error, yet in the BELBIC the steady-state position error eventually decreases. Unlike the PID controller, learning the dynamics through on line implementation causes the BELBIC to track the reference signal inaccurately at the beginning of the experiment (shown in [17]). Despite the fact that the initial weights are all set to zero, the BELBIC rapidly learns the dynamics of the plant without any off-line training. During transient states, a slight overshoot observed in the control signal of the BELBIC since the servo-valve draws more current; however, in the PIDbased controller no such change realized. As the BELBIC passes on to a steady state, the control signal becomes uniform and smooth, which is an important advantage in practical use, especially in high power systems such as EHS systems. The energy consumption of the BELBIC is about the same as the PID controller, whilst the BELBIC has less tracking error. The BELBIC tracks the reference signal with very low error in comparison with the PID controller. The BELBIC displays good robustness to a change in the dynamics of the system, an acceptable overshoot and a good tracking ability (compared to the PID [18]). A main advantage in the performance of the controlled EHS system is in the high degree of the adaptability of the control system and the robustness of the performance with respect to the initial error in relation to modeling and identification (even with a total lack of knowledge about the system model) [17, 18].

\subsection{Problems in intelligent control systems design}

Modern control objects are complex dynamic systems that characterized by information uncertainty of model structures and control goals, a high degree of freedom and essential nonlinearities, instability, distributed sensors and actuators, high level of noise, abrupt jump changes in structure and dynamics, and so on. It is the typical information resources of unpredicted control situations. The structure design of robust advanced control systems for unpredicted control situations is the corner stone of modern control theory and systems. The degree to which a control system deals successfully with above difficulties depends on the intelligent level of advanced control system.

In Step I of developed design technology, we focus the main attention on the description of particular results of KB design and simulating intelligent control systems with essentially nonlinear $\mathrm{CO}$ with a randomly time-dependent structure and control goals. In this case, the aim of this step is to determine the robustness levels of control processes that ensure the required reliability and accuracy indices under the conditions of uncertainty of the information employed in decision-making (learning situations).

For Step 2, the description of the strategy of robust structure's design of an intelligent control system based on the technologies of quantum and soft computing given. The developed strategy allows one to improve the robustness level of fuzzy controllers under the specified unpredicted or weakly formalized factors for the sake of forming and using new types of self-organization processes in the robust KB with the help of the quantum computing methodology. A particular solution of a given problem obtained by introducing a generalization of decision-making strategies in models of fuzzy inference in the form of a new quantum fuzzy inference (QFI) on a finite set of fuzzy controllers designed in advance [19].

The basis for the development of control systems is the proportional-integral-differentiating (PID) controller, which used in 70\% of industrial automation, but often does not cope with the control task and works very poorly in unforeseen situations. Fuzzy controllers allow to partially expanding the scope of PID controllers by adding production logic rules and partially adapt the system. The combined use of genetic algorithms (GA) and a fuzzy neural network made it possible fully adapt the system, but it takes time to train such a system, which is critical in emergency and unforeseen situations. Modeling the optimal training signal makes it possible to create partial self-organization in the system due to the formation of optimal trajectories of the gain of the PID controller. The application of quantum computing and, as a particular example, quantum fuzzy inference (QFI) allows increasing robustness without spending a temporary resource in on line. 


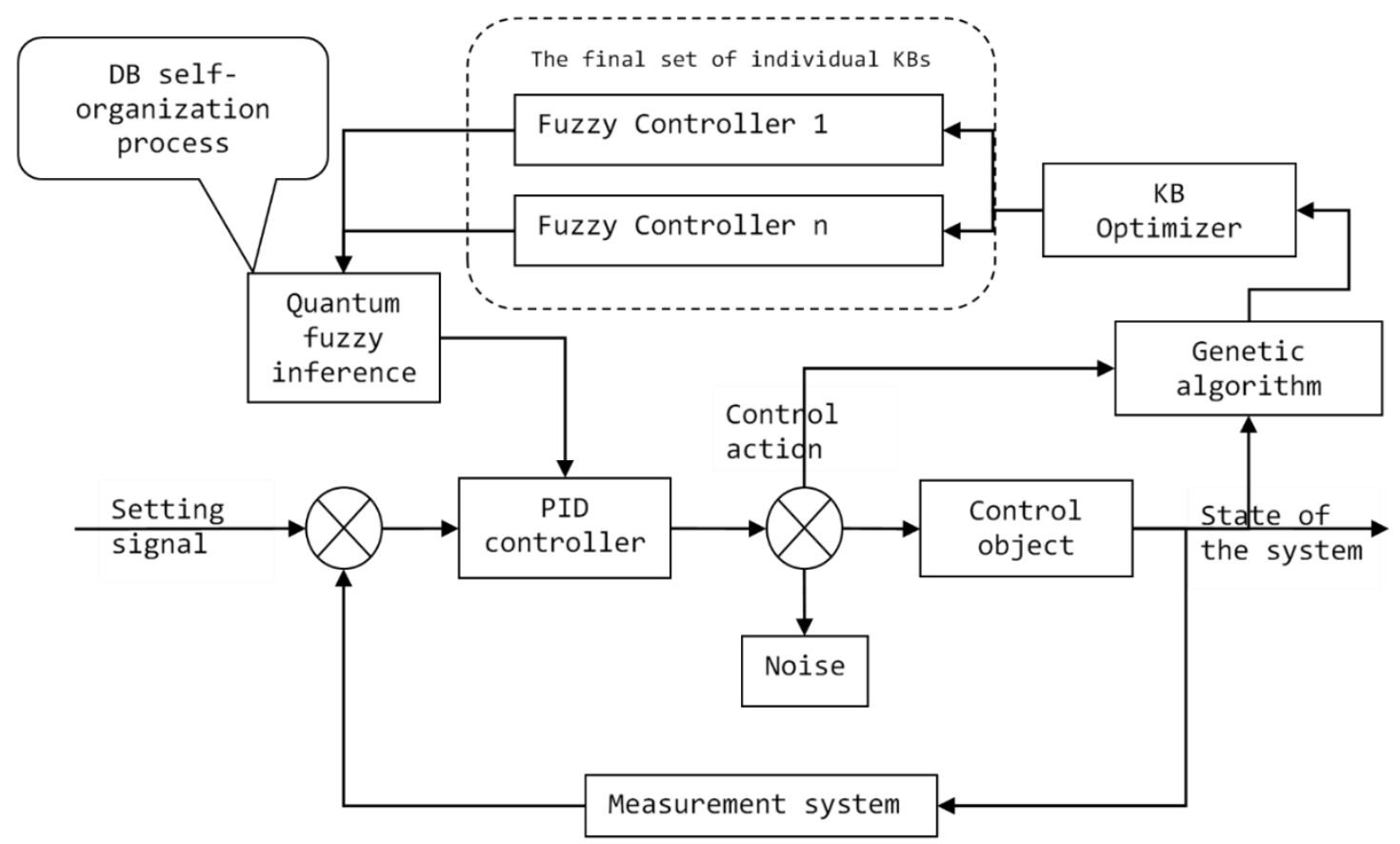

Figure 6. Intelligent control system including quantum fuzzy inference

The Fig. 6 shows ICS structure with the combination of several fuzzy regulators and the quantum fuzzy controller. The main problem in the development and design of this structure that it is very difficult to design a globally good and robust control structure for all possible cases, especially when the system works in poorly predictable situations. One of the best solutions is the formation of a finite number of knowledge bases of a fuzzy controller for a variety of fixed control situations. The goal of a quantum regulator is to combine the knowledge bases obtained with the help of the soft computing optimizer knowledge base into self-organizing quantum fuzzy regulators. The QFI model uses private individual knowledge bases of the fuzzy controller, each of which designed using SCOptKB ${ }^{\mathrm{TM}}$ and QCOptKB ${ }^{\mathrm{TM}}$ toolkits.

Box "Kansei / Kawaii / Affective engineering" (Fig. 1) forming the knowledge about fillings of human being operator and concentrate the attention on control goal. KBs of fuzzy controllers and cognitive controllers designed with $\mathrm{SCOptKB}{ }^{\mathrm{TM}}$ toolkit using objective information of control object response from measurement system in feedback loop and affective response and will of human being operator described with new type of computational intelligence technology. The main performance of Part I to show the description of emotion estimation in Box "Kansei / Kawaii / Affective engineering" and the introduction of physical interpretation of quantum interference in cognition as quantum models of patterns.

Example. In order to clarify the difference in the definition of emotions / feelings used in [20], Figure 7 illustrates concrete examples. In the Figure 7, there is stimulus A and a bodily state that evoke the "Flight" action, whereas a stimulus B and a bodily state activate the "Flight" action. In this case, the emotional state that stimulus A and the bodily sate cause is labeled as "anger," and the emotional state caused by the stimulus B and the bodily sate is labeled as "Fear." This definition directly connects emotions to the somatic marker hypothesis, which means that the emotion should generated by considering internal appraisal, external appraisal, and decision-making mechanisms. 


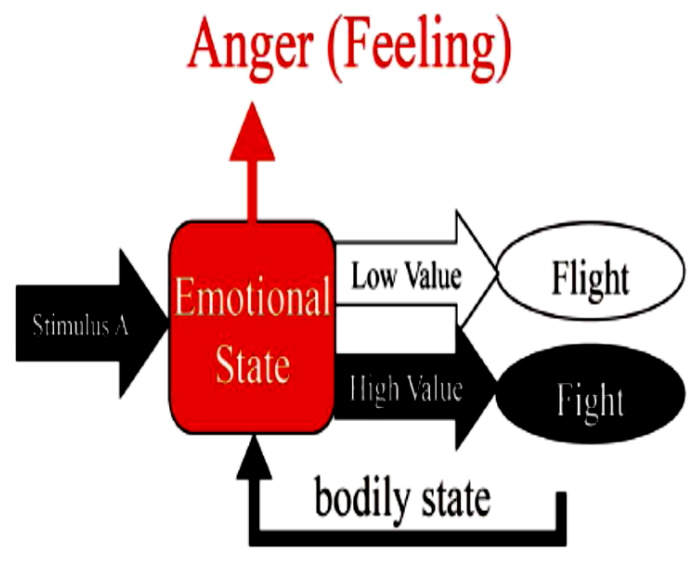

(a)

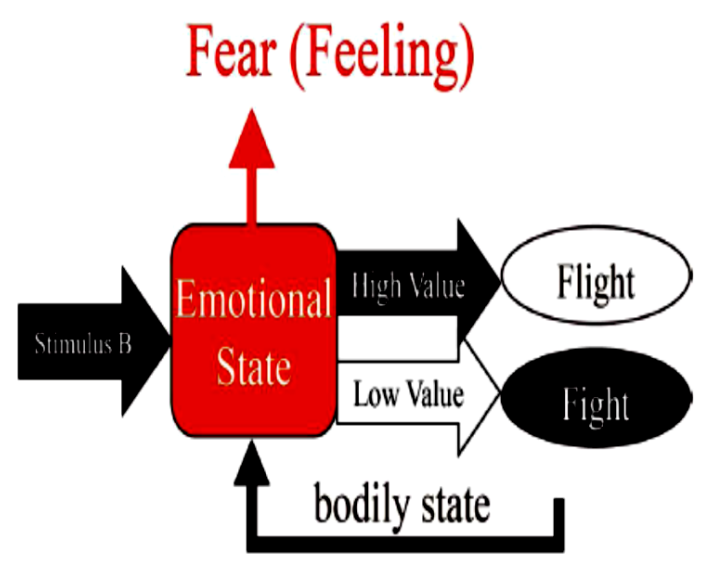

(b)

Figure 7. Illustration of "anger" and "fear", which highlights the difference: (a) emotional feeling of anger, and (b) emotional feeling of fear

However, the ICS structures do not have a specialized software module to describe and implement the processes of adaptation and learning of the control system to the qualitative characteristics of human being operator behavioral responses. Proven in a wide class of areas of soft computing (genetic algorithms, fuzzy logic and fuzzy neural networks) and computational technology in the form of intelligent tools (Computational Intelligence Toolkit), allows to design an intelligent cognitive control system that has the required qualities.

The cognitive processes of non-verbal communication in the human brain (see Fig. 8) modeling on such a level: they explain the correlation between what the human perceives from the clinician's communication, and what the human in turn communicates. The underlying condition of an observed human can then inferred from the recorded interaction with the clinician.

Figure 8 describes general structure of intelligent cognitive robotic control with "brain-computer-robotdevice" neurointerface and affect decoding controller based on Kansei / Affective Engineering and its cognitive computing technology.

Kansei / Affective Engineering technology and its cognitive computing toolkit include qualitative description of human being emotion, instinct and intuition that used effectively in design processes of smart / wise robotics and intelligent mechatronics as example robot for service use [11,21] and robotic unicycle (see, for example below).

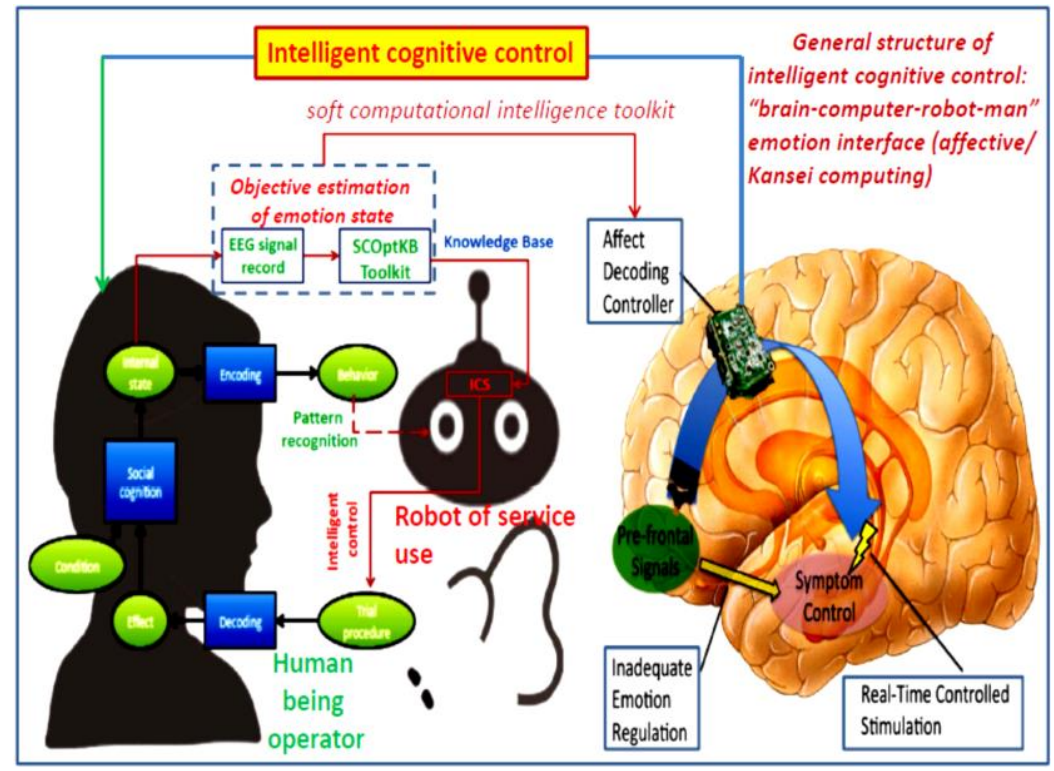

Figure 8. General structure of intelligent cognitive human-robotic interaction control 
Remark. According to general definition Kansei Engineering (Japanese: 感性工学 kansei kougaku, emotional / affective engineering) aims at the development or improvement of products and services by translating the customer's psychological feelings and needs into the domain of product design (i.e. parameters). Mitsuo Nagamachi, Ph.D, Professor Emeritus of Hiroshima University founded it. Kansei Engineering parametrically links the customer's emotional responses (i.e. physical and psychological) to the properties and characteristics of a product or service. In consequence, products can design to bring forward the intended feeling. The main part the mammalian brain is responsible for emotional processes and called the limbic system. The computational models of the amygdala and orbitofrontal cortex are the main parts of the limbic system recently introduced for the first time. Therefore, Kansei result is a synthesis of sensory brain cognitive qualities. For example, it has argued that emotion, pain and cognitive control functionally segregated in distinct subdivisions of the cingulate cortex of brain (see, Appendix 1).

The processes depicted in Fig. 8 represent incredibly complex, non-smooth, and non-linear mappings and representations, which indicates that it will be suitable to use a deep neural network [4] approach. In this paper we concentrate our attention on description on the box "Objective estimation of emotion state" of Fig. 8 for design of knowledge base of robot for service use [11,21]. Robots for service use can practically implemented into current education and therapy interventions for children ASD.

\subsection{Social human-robot emotion interaction and application}

The Center for Disease Control (CDC), has recently announced that the incidence of autism is 1 in every 59 children. There has been a growth rate of $250 \%$ during the last 15 years. Autism is now emerging as a public health priority. ASD occurs in all racial, ethnic, and socio-economic groups. However, the incidence is five times more common among boys than among girls [22,23]. In particular, according to the Centers for Disease Control and Prevention, one in every 68 children (1:42 boys, 1:189 girls) ASD [24]. Individuals with ASD exhibit impairments in three key areas: (a) communication, (b) social interaction, and (c) restricted interests and repetitive behaviors. The American Psychiatric Association recently redefined qualifiers for ASD, citing levels of severity, the impact deficits key areas have on the quality of life and the amount of support needed, beginning with Level I (less support, formerly included diagnosis of Asperger Syndrome, Pervasive Developmental Delay-Not Otherwise Specified), Level II (moderate support), and Level III (most support).

The schema at the Fig. 9 shows how the child-robot interaction loop and the software modules are used by the robot to interact with the child: The Robot Intelligent Module (RIM) and the Behavior Manager (BM).

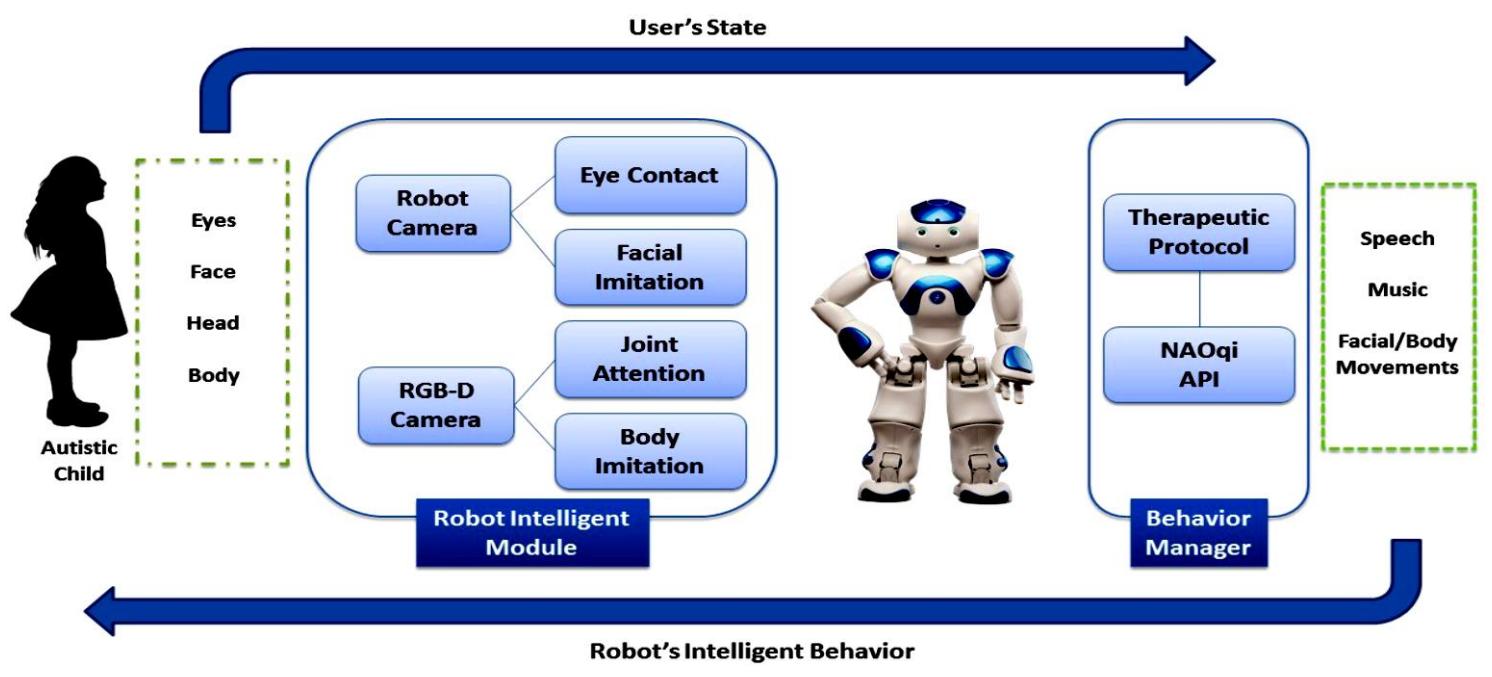

Figure 9. Artificial Intelligence System for Robot-Assisted Treatment of Autism

The RIM is composed of four components: head pose, body posture, eye contact, and facial expression. The BM consist of two components: the treatment protocol and the NAOqi API [25].

Although robot therapists are better than human therapists in these three areas, they are not yet perfect. The robots for the therapies come at a high cost. Robokind, the company that makes Milo, reports that its robot has an initial cost of $\$ 5,000$ plus an additional cost of $\$ 4,500$ every year after. This is lower than the $\$ 29,000$ cost of human therapy, but Robokind's cost estimates are still too optimistic. A 2015 study found that parents 
have a preference that there is a human complementing the robot in autism therapy. If humans have to complement robots in robot-assisted therapy, the cost might even be higher than the human-led therapy cost of $\$ 29,000$ per year.

As you can see at the Figs 10 and 11, robots used in autism therapy.
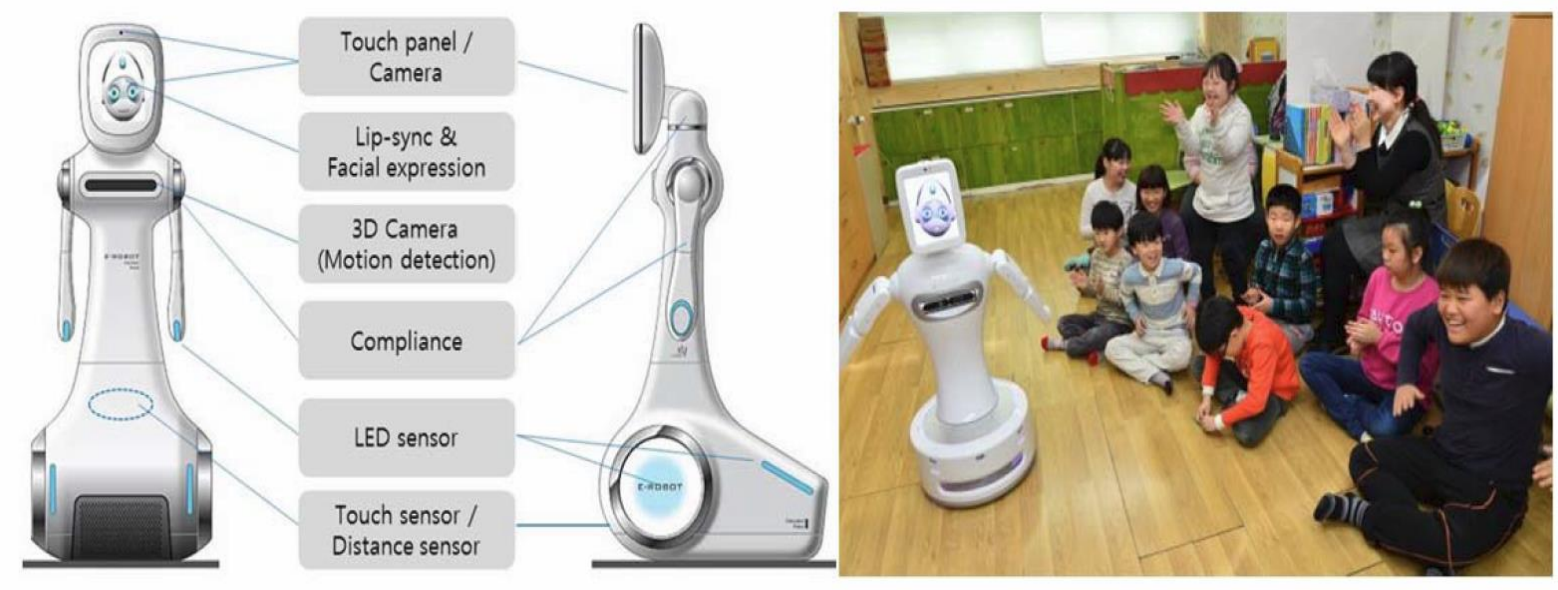

Figure 10. ROBOJJANG developed by Robocare Co., Ltd.

Interacting with robots can be particularly empowering for children with ASD, because it may overcome various barriers experienced in face-to-face interaction with humans. Moreover, robot assisted interventions can be tailored to the needs of the specific child and can be used in an identical manner as often as needed.
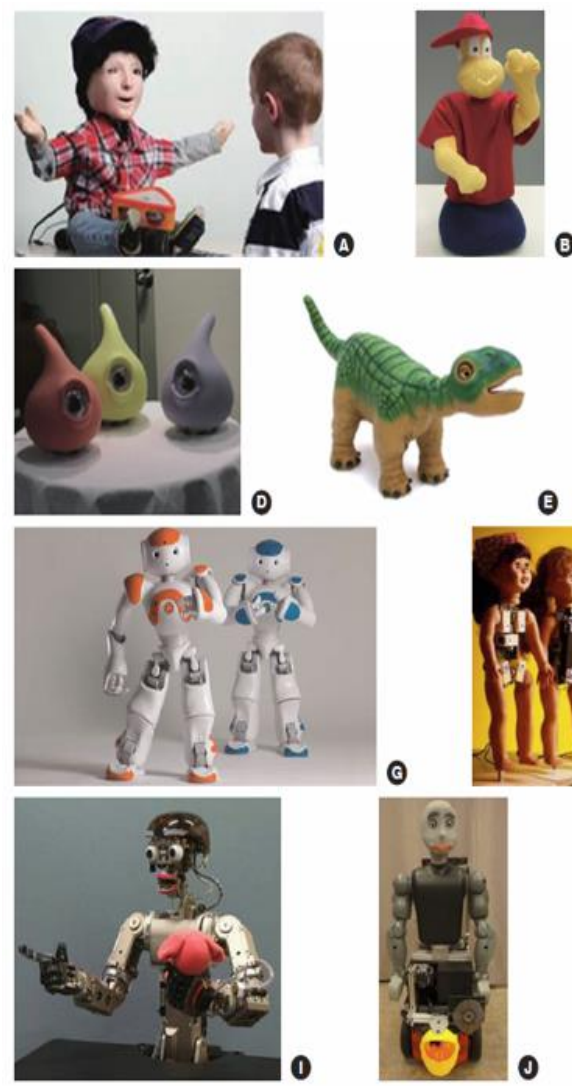

$\theta$
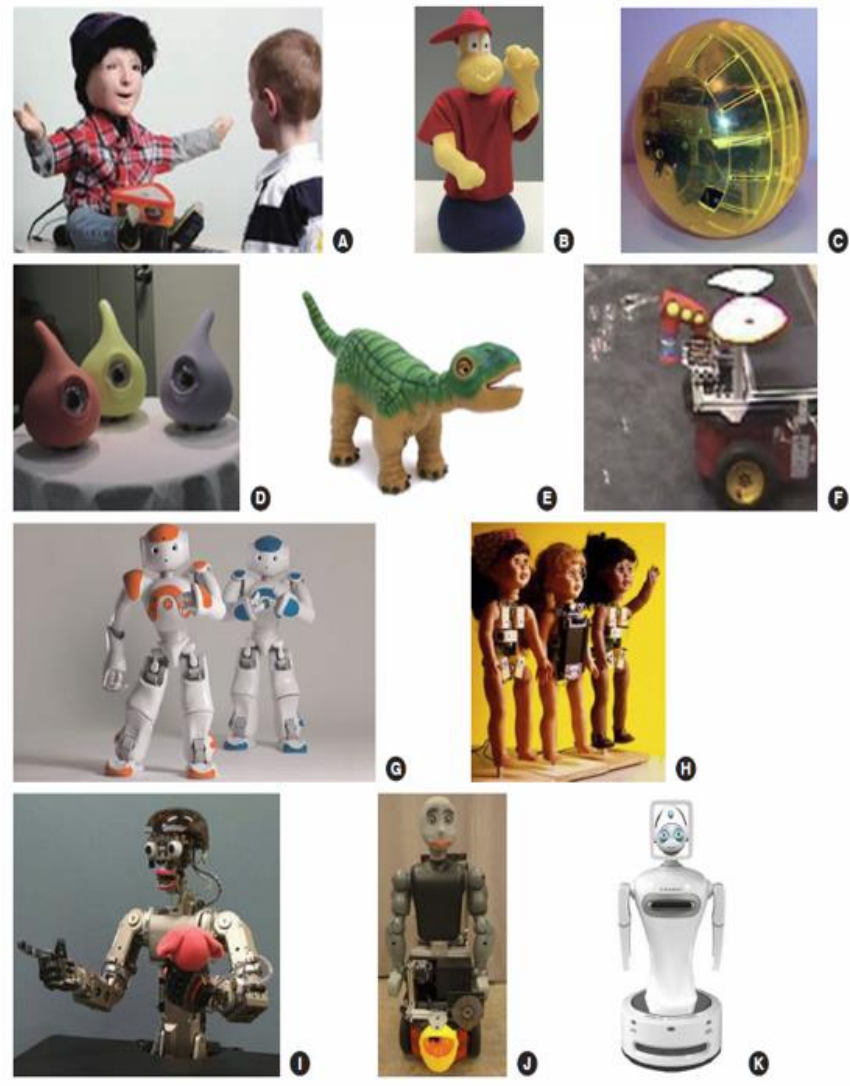

Figure 11. Robots used in autism therapy

Figure 11 shows the robots used in autism therapy all around the world:

- Kaspar (courtesy of the Adaptive Systems Research Group, University of Hertfordshire, UK),

- Tito (courtesy of F. Michaud), 
- Roball (courtesy of F. Michaud),

- Muu (courtesy of M. Okada, Toyohashi University of Technology, Japan),

- Pleo (courtesy of Innvo Labs Corporation),

- Bubble blower (courtesy of D. Feil-Seifer),

- Nao (courtesy of Aldebaran),

- Robota (courtesy of A. Billard), (I) Infanoid (courtesy of H. Kozima),

- Bandit (courtesy of M. Mataric, USC, USA), and (K) Robojjang (courtesy of Robocare Co., Ltd.).

\subsection{Therapy of the autism using the intelligent cognitive system}

It is believed that effective therapy for autism is extremely expensive. It is not because it is complicated, but because the small number of the specialists who own behavioral techniques. There is a situation when the majority of families do not have access to the necessary treatment [26].

Remark. This work is a continuation of the development of a cognitive-intelligent system for the diagnosis, adaptation and training of autistic children (CISDAEAC). A more detailed description of the CISDAEAC may be found in [27-29].

The main part of this cognitive-intelligent system is the data processing module (see Fig. 12). It represents the structure of a child's interaction and training program through the application of fuzzy logic.

The data processing module is designed to extract the EEG based on a cognitive helmet, process and filter the received signal, create a cognitive process training program on the platform, diagnose problems with the child's work with the system and evaluate the operator's response to the tasks generated by the training module.

CISDAEAC designed to extract process and formulate a learning program based on cognitive processes, in particular, EEG signals, adaptation of autistic children to society and training in basic household skills. One of the tasks of this work is the processing of the EEG signal, based on the recognition of emotions, and forming an encephalographic portrait of the child on the next step.

Before working with the system, a detailed assessment of the current level of social interaction of the child, revealing the difference between the difficulties in acquiring. Next creating a minimum training package to determine the starting point consisting of basic logical tasks. To receive feedback, the Emotiv EPOC+ cognitive helmet used, which allows recording the brain activity signal and transferring it to the data processing module.

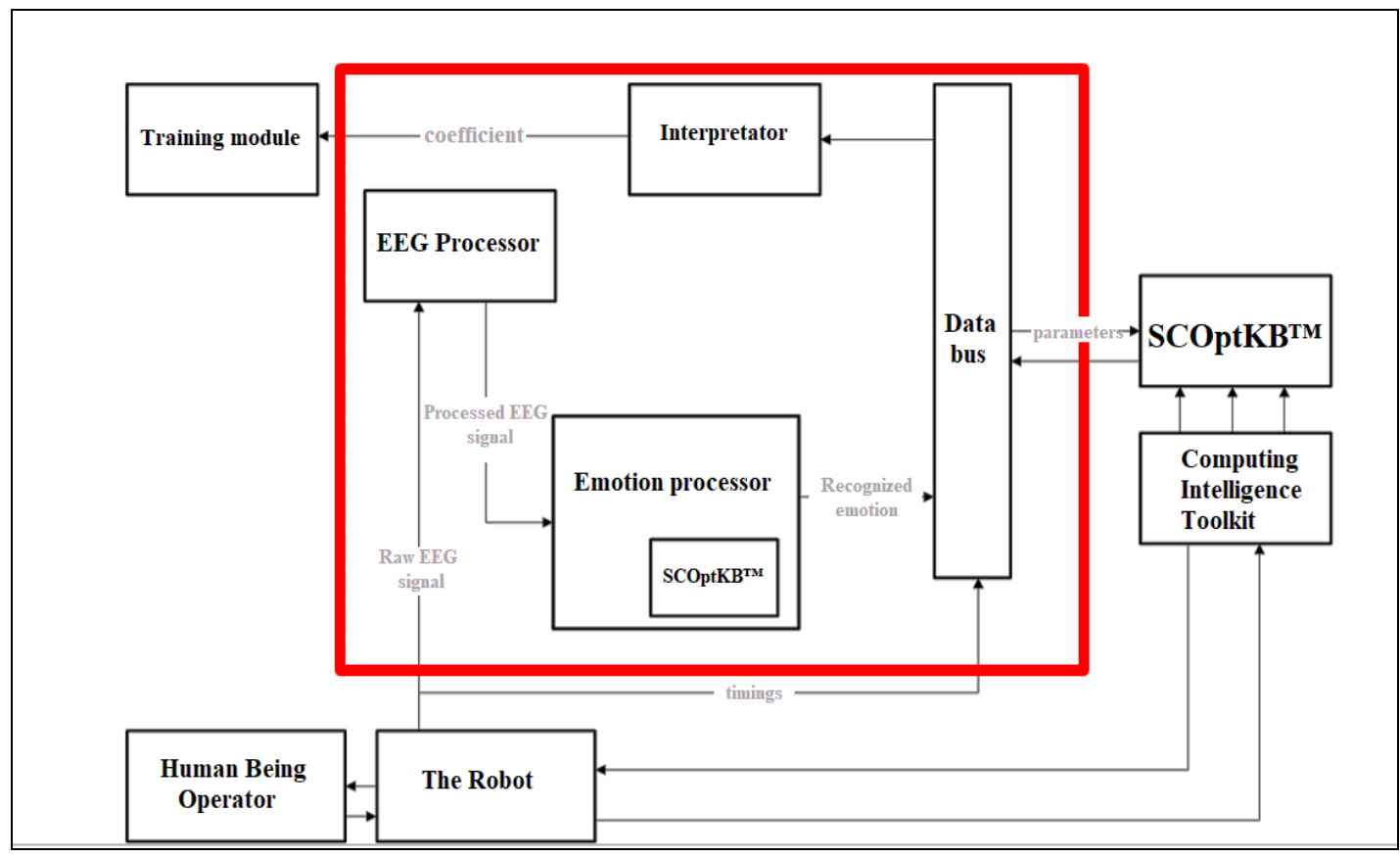

Figure 12. CISDAEAC Data processing module 
Next, by the EEG signal, evaluating the child's reaction to the interaction with environment, and monitoring parameters for solving the tasks. Then forming the strategy of learning using the technology of soft computing. The signal from the EPOC signal recognition unit, the decision time, the correctness of the solution and the task identification number are using as the input data. After this, the system sets the appropriate coefficients for adjusting the training program.

\section{EEG signal processing}

\subsection{Features of experimental EEG registration and informative parameters of the patient's condition}

The electroencephalogram (EEG) of the human operator can be used as a biometric parameter, since the brain activity is individual. It is made unique by synchronized activity of groups of neurons that process the same signals to form metastable group. Signals corresponding to one external stimulus or cognitive event trigger synchronized activity of neurons grouped together. A certain level of synchronization is maintained at rest state. Synchronized neuronal activity is observed on the EEG.

Recording EEG signal is a contact and long-term procedure, since the electrical activity of the brain is a value extended in time, and the data cannot be recorded for a long time because of the nonlinear distortions of the EEG signal appear at large intervals. The nonlinearity of the signal can be solved by a series of short measurements, during which the signal can be considered linear. Emotiv EPOC+ cognitive helmet was used for recording the brain activity (see Fig. 13).

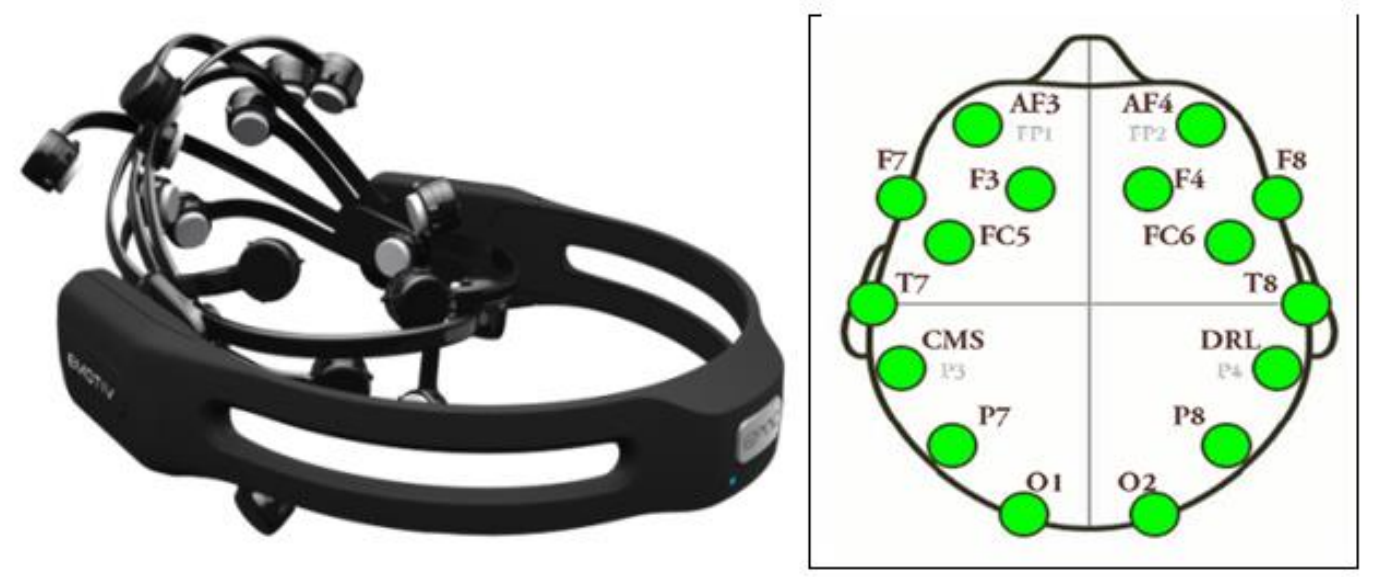

Figure 13. Emotiv EPOC+ cognitive helmet

EPOC has 14 electrodes, which are passive sensors that allow register electromagnetic signals. Sensors are attached to the surface of the skin (non-submersible, non-invasive interface). Figure 8 presents the structure of Emotiv EPOC+, consisting of channels AF3, F7, F3, FC5, T7, P7, O1, O2, P8, T8, FC6, F4, F8, AF4 and two reference sensors CMS/DRL, which purpose is to receive and filter bioelectric signals of muscle activity from the EEG signal.

Figure 14 study selection and multivariate modeling. 

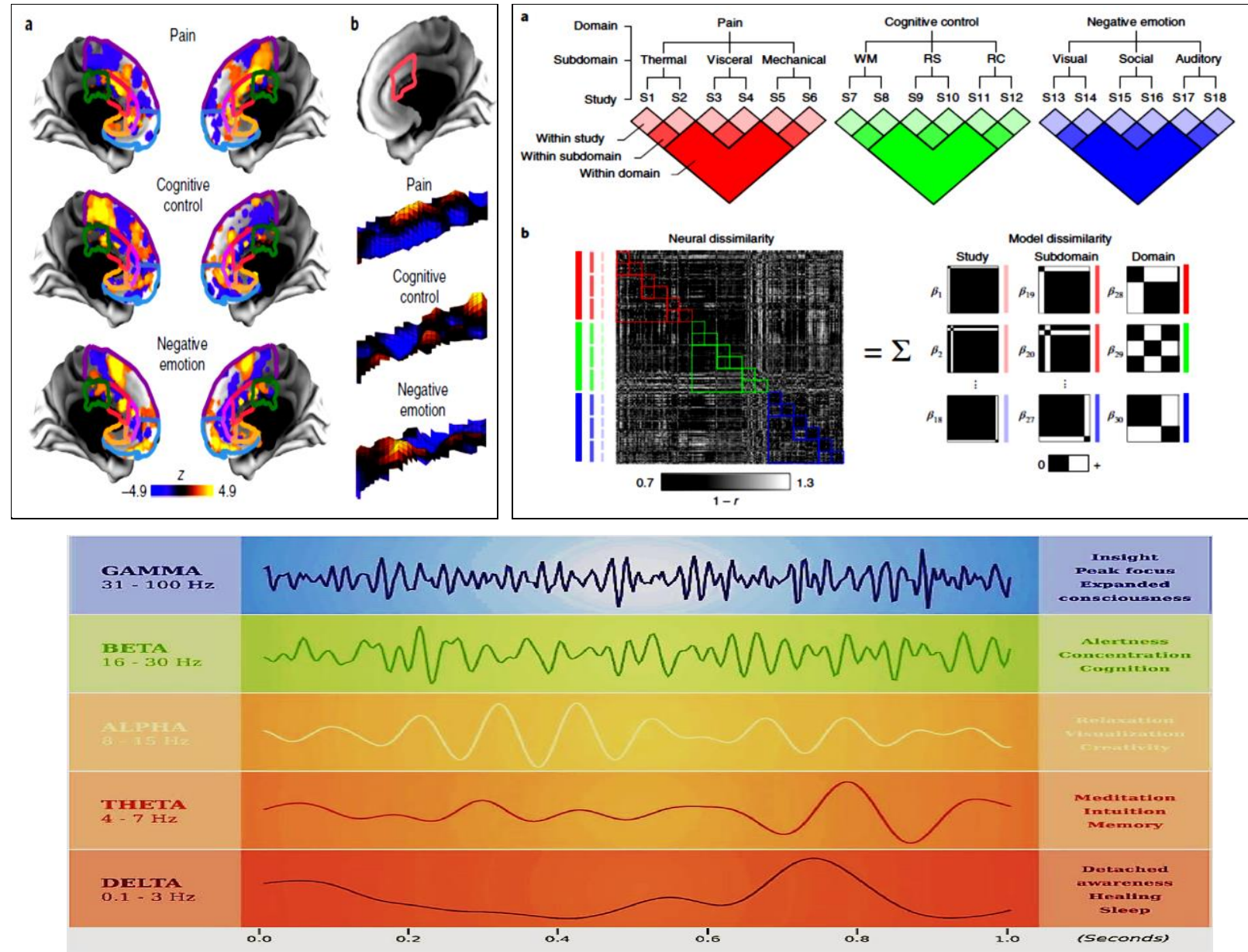

Figure 14. Hierarchical structure of studies and tasks

Remark. On Fig. 14 a, Hierarchical structure of studies and tasks. Dendrograms convey theoretical groupings of fMRI activity at levels of study (level 1: studies S1-S18), subdomain (level 2: thermal, visceral, mechanical, working memory (WM), response selection (RS), response conflict (RC), visual, social, and auditory), and domain (level 3: pain, cognitive control, and negative emotion). Colored regions illustrate model based partitioning of neural similarity into components that generalize across subjects (unique to a study, top 18 squares), studies (unique to a subdomain, middle nine squares), and subdomains (unique to a domain, bottom three regions). b, Decomposing multivariate pattern similarity into study-, subdomain-, and domainspecific components.

The matrix in the left panel shows the dissimilarity of fMRI patterns across all subjects $(n=270)$ in the entire medial frontal cortex. Each row represents one individual participant and each element the dissimilarity (1-Pearson' s correlation coefficient) in brain activity patterns for two individuals. Colored bars to the left indicate corresponding levels in the functional hierarchy. The right panel shows how the observed neural dissimilarity across pairs of images from the 18 studies is modeled as a weighted summation of theoretical dissimilarity matrices constructed according to study (18 parameters), subdomain ( 9 parameters), and domain (3 parameters) membership, in addition to a constant term (not shown).

The supplied software allows in on line to receive, recognize and register the EEG signal from the helmet [31]. As part of the solution of the problem it is necessary to obtain the most informative fragments of the signal. Frequency rhythms of EEG are distinguished for the analysis. The concept of frequency rhythm determines the type of electrical activity corresponding to a certain state of the brain which boundaries of the frequency range are determined (see Fig. 15). 


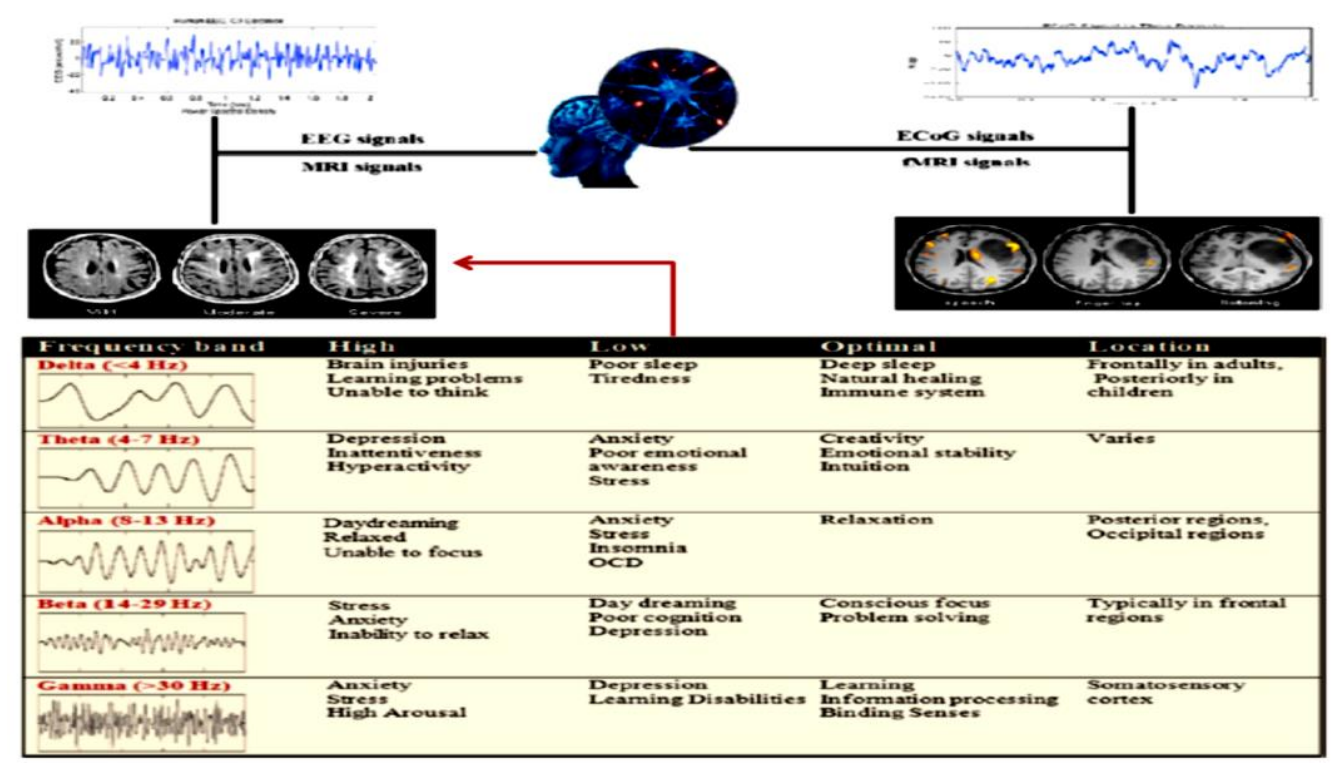

Figure 15. EEG frequency rhythms

This involves the decomposition of the EEG signal into frequency components, which is achieved by fast Fourier transforms (FFT), which returns for each frequency buffer a complex number containing the amplitude and phase.

$$
X_{k}=\sum_{n=0}^{N-1} x_{n} \cdot e^{-i 2 \pi k n / N}
$$

where $\mathrm{N}$ is the number of time samples, $\mathrm{k}=0, \mathrm{~N}$ is 1 is the current frequency, $\mathrm{n}=0 \ldots, \mathrm{N}-1$ is the current sample, $\mathrm{x}_{\mathrm{n}}$-input samples in the time domain, $\mathrm{x}_{\mathrm{k}}$-output samples in the frequency domain.

\subsection{Definition of emotional arousal}

A well-known marker of cognitive processes is the restructuring of brain rhythms which occurs in the superficially recorded human EEG. Strong emotional experience, as a form of cognitive activity, can lead to inhibition of other mental processes, realization of behavioral appropriate reactions, violation of conscious control over actions, as a result of which uncontrolled actions can be committed [32]. States arise against the will, conscious control over their actions is not possible. The occurrence of such situations can lead to a critical error in the control loop [33].

Therefore, the first task was to determine the level of emotional arousal of the human being operator.

Figure 16 identifying latent brain representations that predict the occurrence of distinct functional domains in each region of interest. 

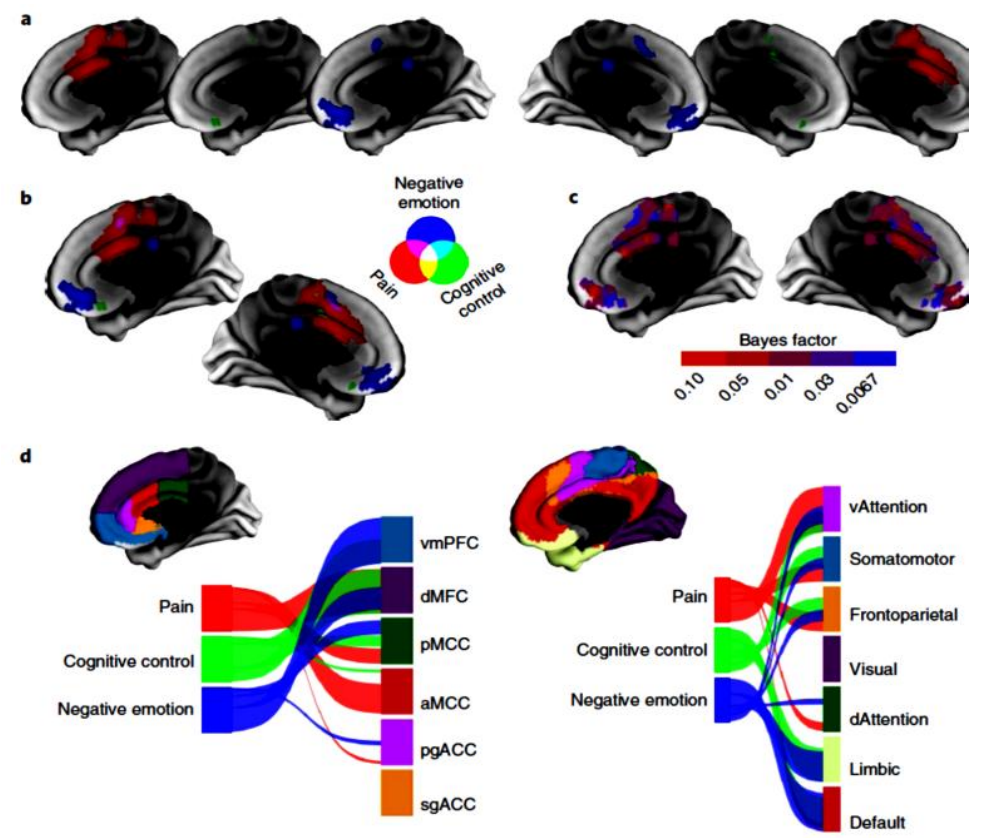

Figure 16. Representational mapping of pain, cognitive control, and negative emotion in MFC

On Fig. 16a, Searchlight maps display where local patterns of brain activity are consistent with domainspecific representation of pain (red), cognitive control (green), and negative emotion (blue; $\mathrm{n}=270$ participants). b, Additive conjunction of searchlight maps, with each domain mapped onto orthogonal dimensions in the red-green-blue (RGB) color space. Overlap between pain and cognitive control is depicted in yellow; overlap between pain and negative emotion is colored magenta. Maps are thresholded at $\mathrm{P}<0.05$, two-tailed, uncorrected cutoff to highlight any possible overlap ( $\mathrm{n} !=! 270$ participants). c, Brain maps of Bayes factors indicating relative evidence against overlap among the three domains at each voxel. Smaller values indicate evidence against overlap; values less than 0.1 are considered strong evidence ( $n=270$ participants). $\mathrm{d}$, River plots depict the similarity between searchlight maps and anatomical parcellation of MFC (left) and functional parcellation of cortical regions from resting-state data48 (right). Line thickness indicates the degree of correspondence between sets. v, Attention, ventral attention; d Attention, dorsal attention. Images are displayed using radiological convention.

\subsection{Experimental results}

During the study, the operator's EEG signal was recorded while in a calm state and in a state of stress. The source signal of each sensor, with a sampling frequency of $128 \mathrm{~Hz}$, is decomposed into frequency rhythms using a discrete Fourier transform.

For visual assessment of differences in emotional states, graphs of the spectral power of signals from AF3, $\mathrm{AF} 4, \mathrm{~F} 7, \mathrm{~F} 3, \mathrm{~F} 4, \mathrm{~F} 8, \mathrm{FC} 5, \mathrm{FC} 6, \mathrm{~T} 7, \mathrm{~T} 8, \mathrm{O} 1, \mathrm{O} 2$ sensors were constructed in the range of significant frequencies from 1 to $50 \mathrm{~Hz}$. (see Fig. 17). 


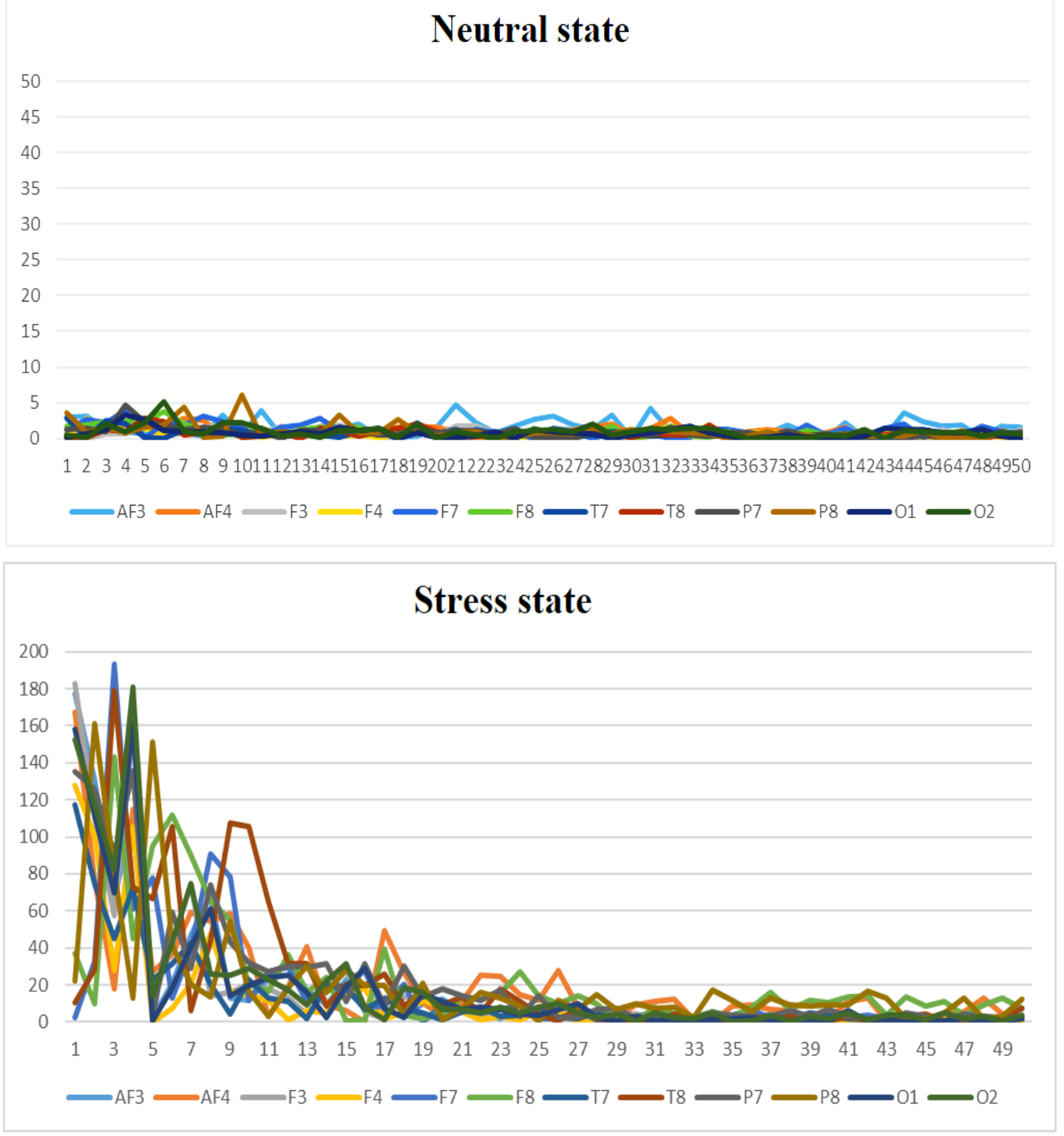

Figure 17. A graph of the spectral power density of the EEG signal obtained by discrete Fourier transform for the state and stress of rest

A comparative analysis of the spectral power of various emotional states for the frontal, temporal, and occipital-parietal lobes of the cerebral cortex has been carried out (see Figs 18, 19 and 20).

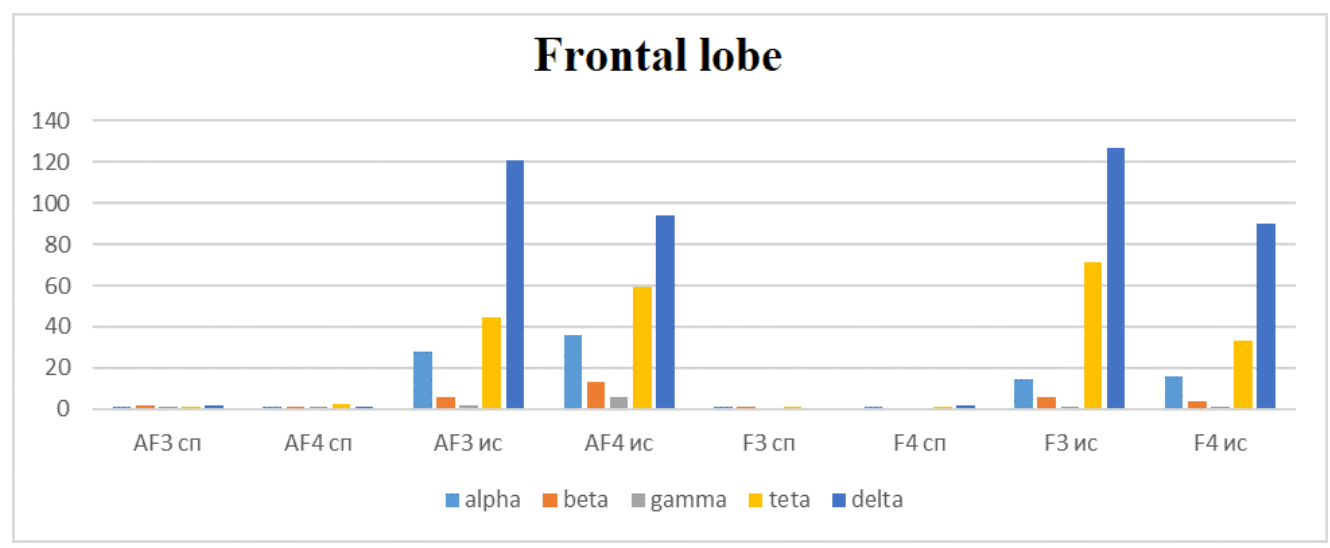

Figure 18. The level of spectral power of the EEG signal for sensors AF3, AF4, A3, A4 for each of the frequencies for two emotional states: calm and fright 


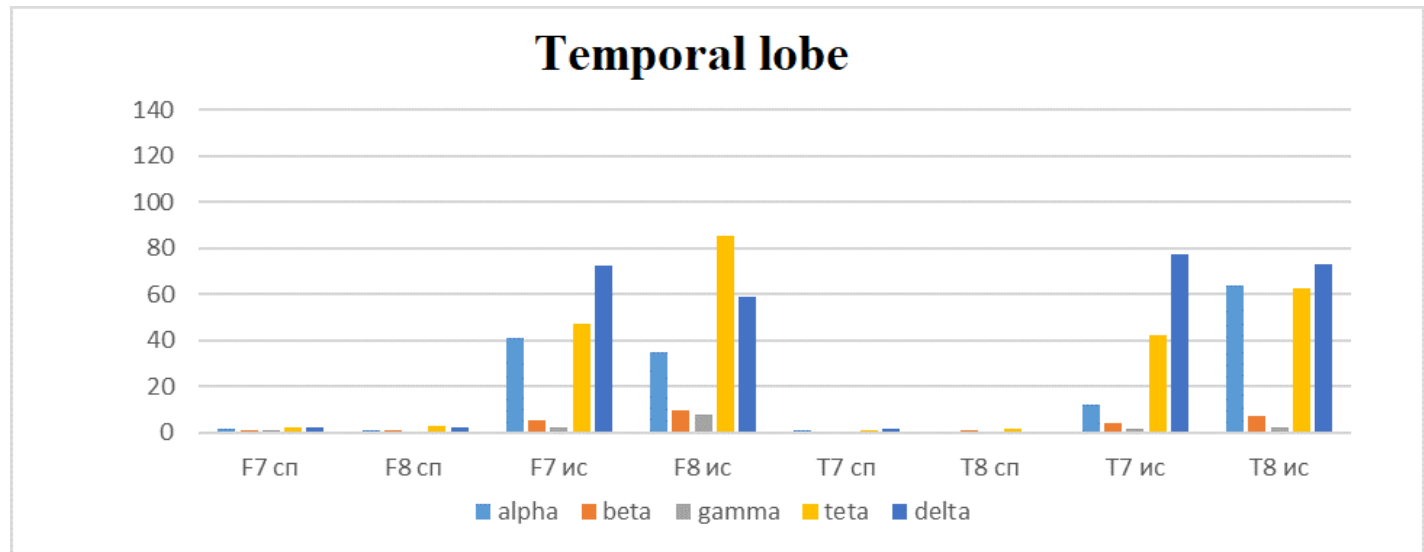

Figure 19. The level of spectral power of the EEG signal for sensors $P 7, P 8, O 1, O 2$ for each of the frequencies for two emotional states: calm and fright

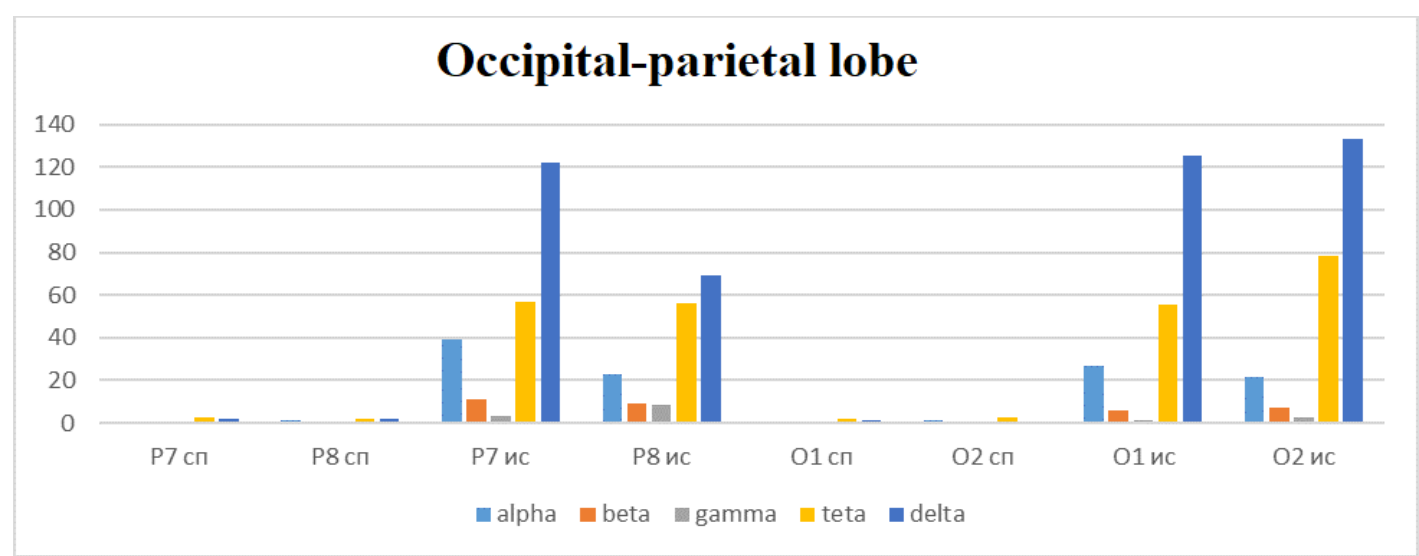

Figure 20. The level of spectral power of the EEG signal for sensors F7, F8, T7, T8 for each of the frequencies for two emotional states: calm and fright

Based on the assessment of the total tonic activity, as well as the values of the total spectral power of the frequency ranges, it was concluded that it is possible to estimate the emotional background of a human being operator.

Figure 21 is demonstrated graphs of signal activation integral accumulation for various emotional states.

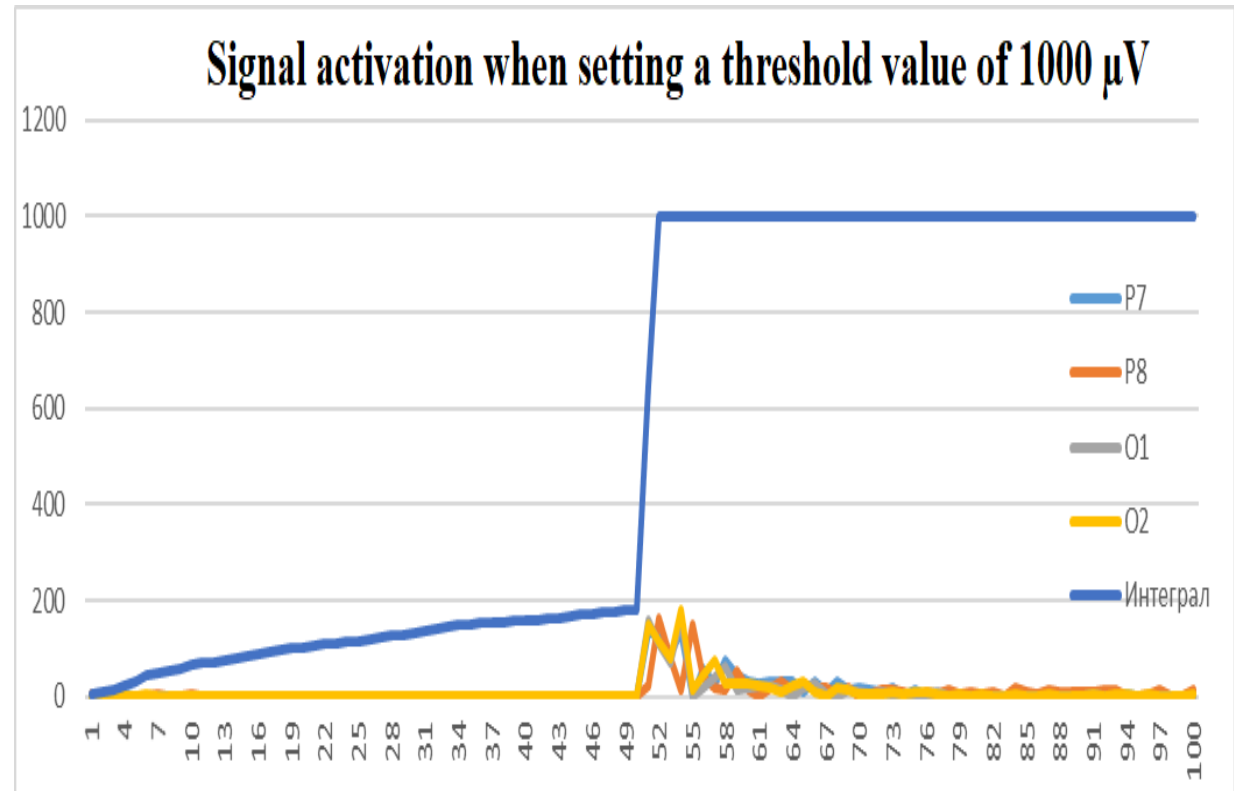




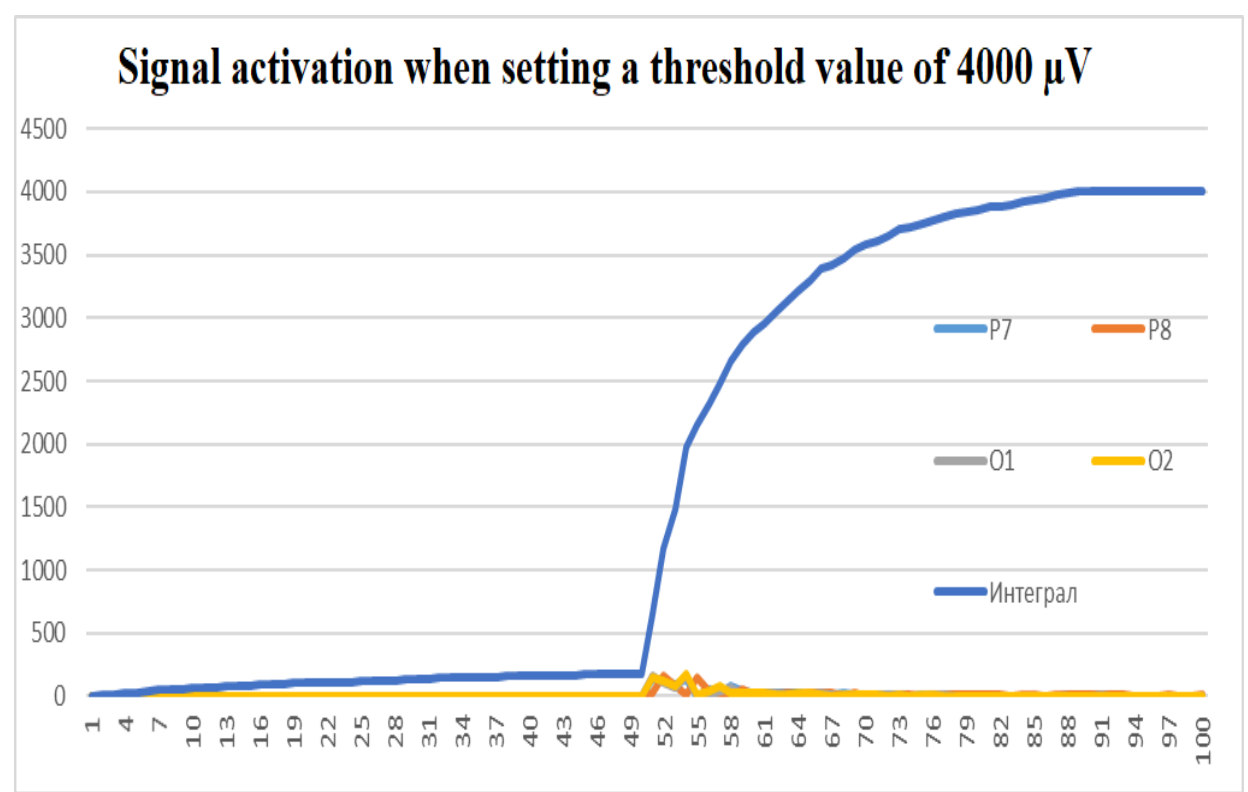

Figure 21. The accumulation of signal activation when setting a threshold value of 1000 and $4000 \mu \mathrm{V}$

At calm state, the signal integral does not exceed $200 \mu \mathrm{V}$, while in a state of emotional arousal a threshold value of $1000 \mu \mathrm{V}$ achieved by one iteration, and for $4000 \mu \mathrm{V}$ in 38 iterations.

The EEG signal registered by the software product using the knowledge base returns the coefficient determining the level of emotional arousal. Based on this coefficient a warning about the level of emotional arousal is displayed on the screen through expert judgment (see Fig. 22).

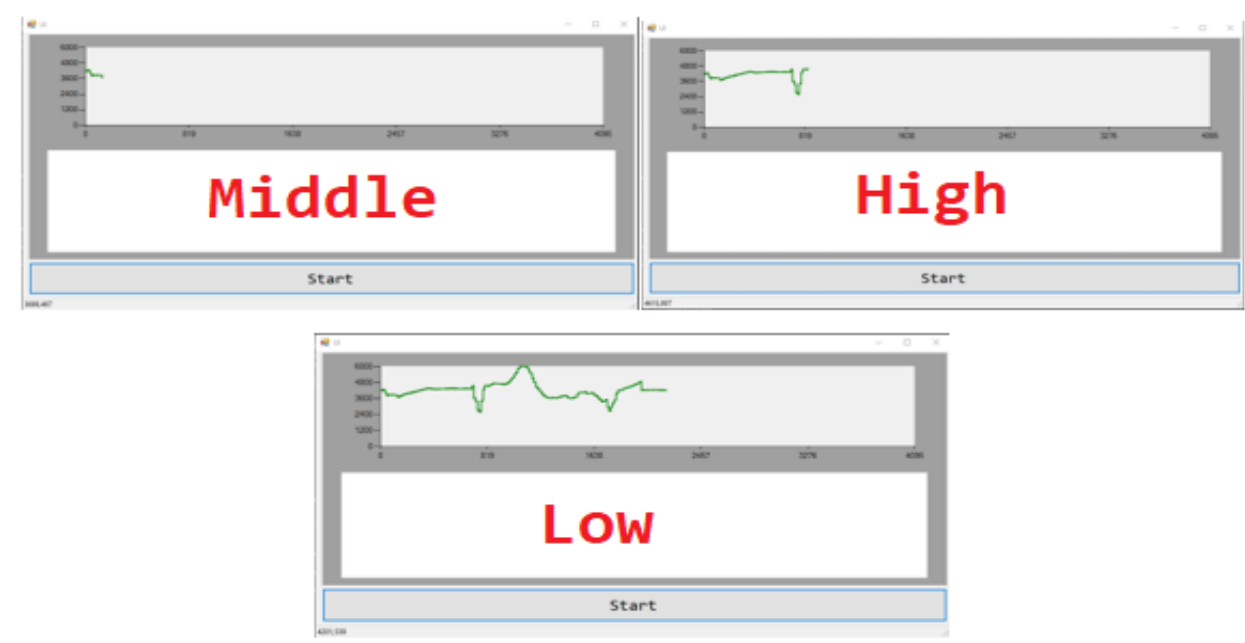

Figure 22. UI of the warning about the level of emotional arousal

\section{Detecting the mental state of a human operator}

First of all, we investigate fear emotion - the marker of valence measurement of emotional states. Fear is realized more clearly, unlike other emotions, and finding its causes is much easier. In the case of autistic children, the emotion of fear is most critical to recognition.

Figure 23 shows a graph of the EEG signal taken by the sensors F3, F4, FC5, FC6 in two emotional states: on the left is fear, on the right is a neutral state. 


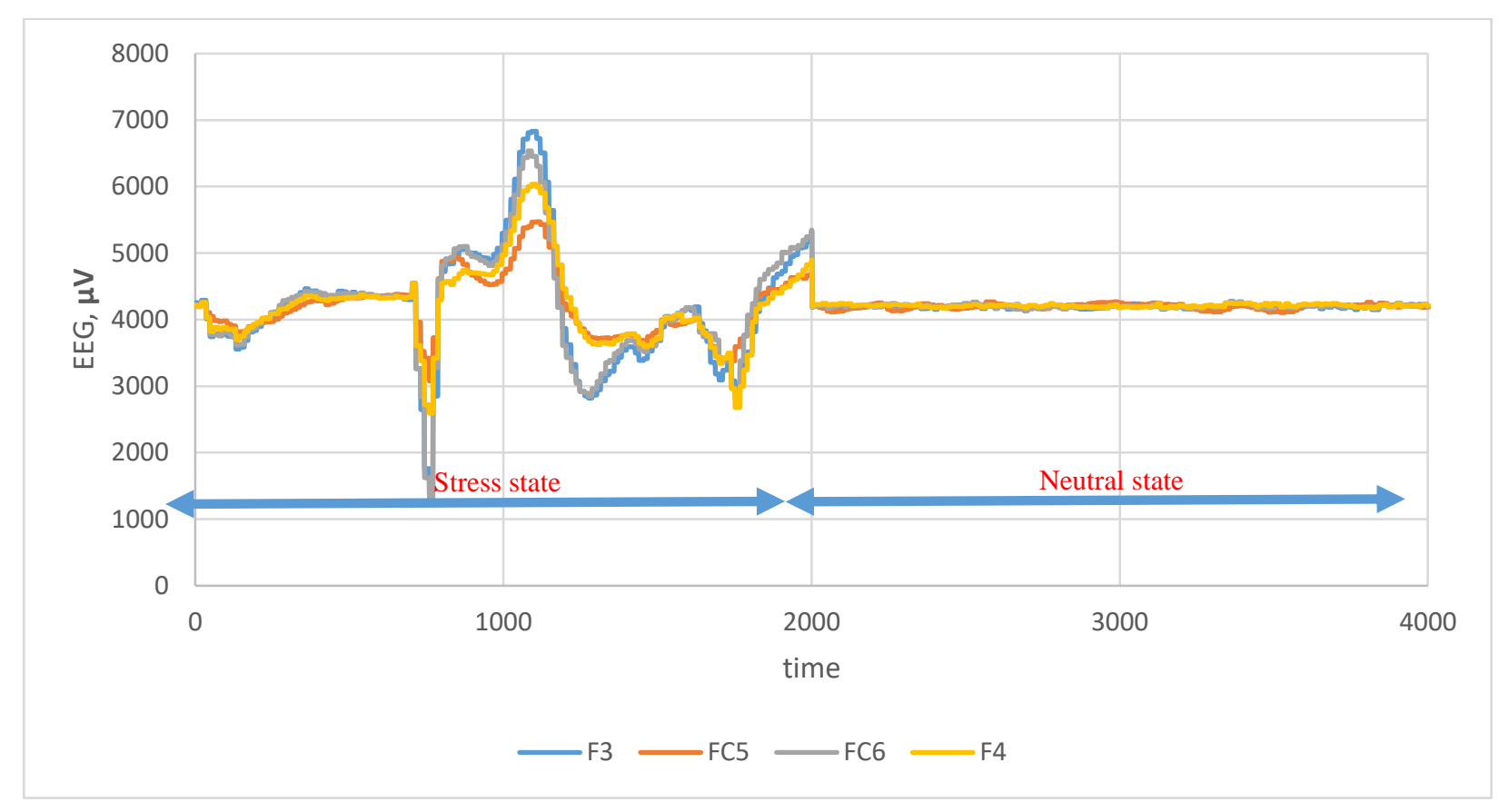

Figure 23. The value of the EEG signal of different emotional states

On the example of the data of the EEG signal values, the implementation of the visual display of the signal is considered, and also these data were used to form the knowledge base. This requires a transition from the representation of the EEG signal as a function of time, to the representation of the signal in the spatial frequency domain. This transition is carried out by decomposing the signal into harmonic components using the Fourier transform. Figure 24 shows a graph of the spectral power density of the EEG signal taken by the AF3 sensor in two emotional states: on the left is fear, on the right is a neutral state.

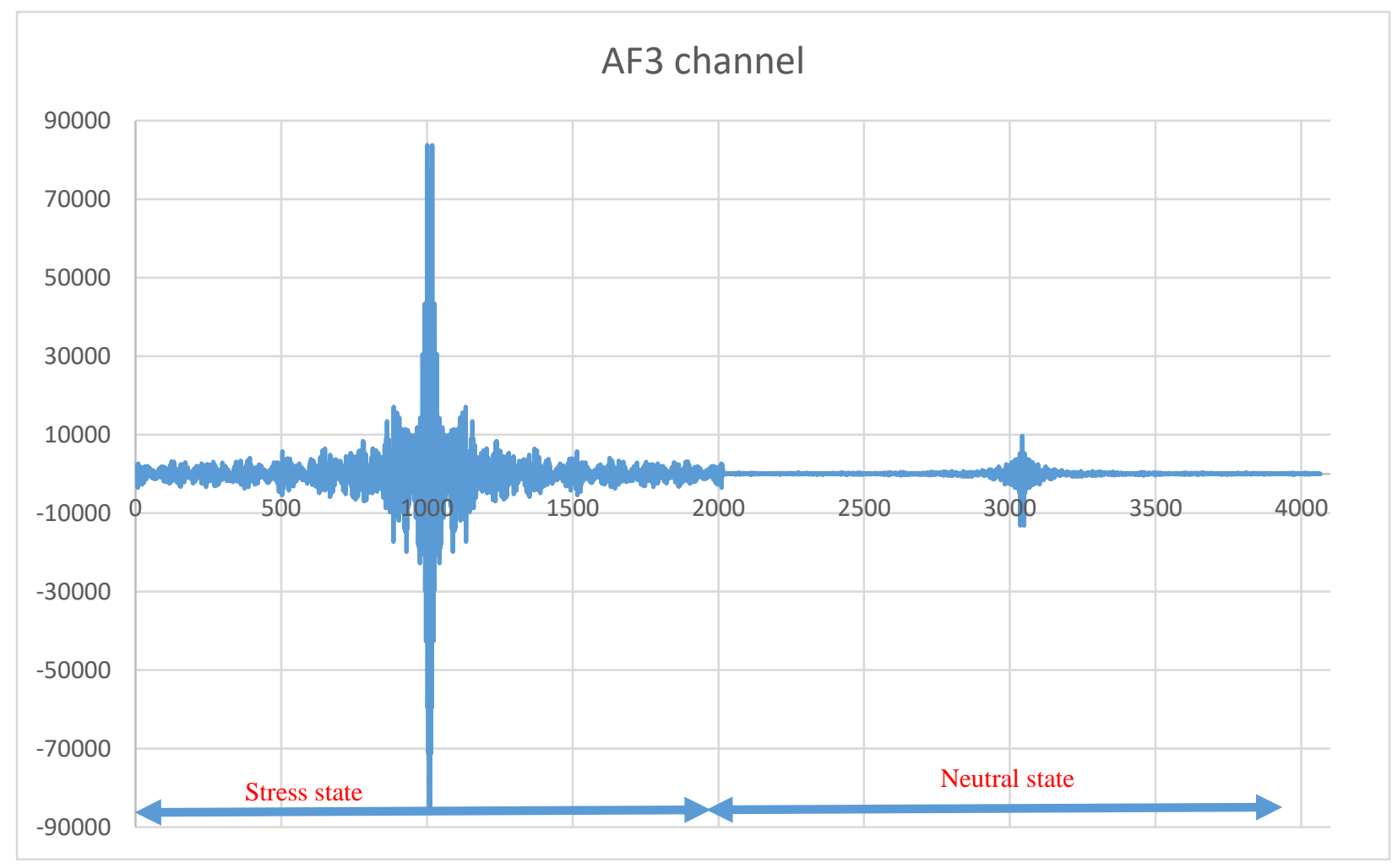

Figure 24. The value of the total spectral power density of the EEG signal for various emotional states

This approach reduces the amount of processed data for visual assessment, makes it possible to quickly classify electroencephalograms. Regardless of emotions sign, it can be generated in both hemispheres of the 
brain, but a number of studies focuses the attention of the anterior sections of the brain in the generation of emotions (see Fig. 23).
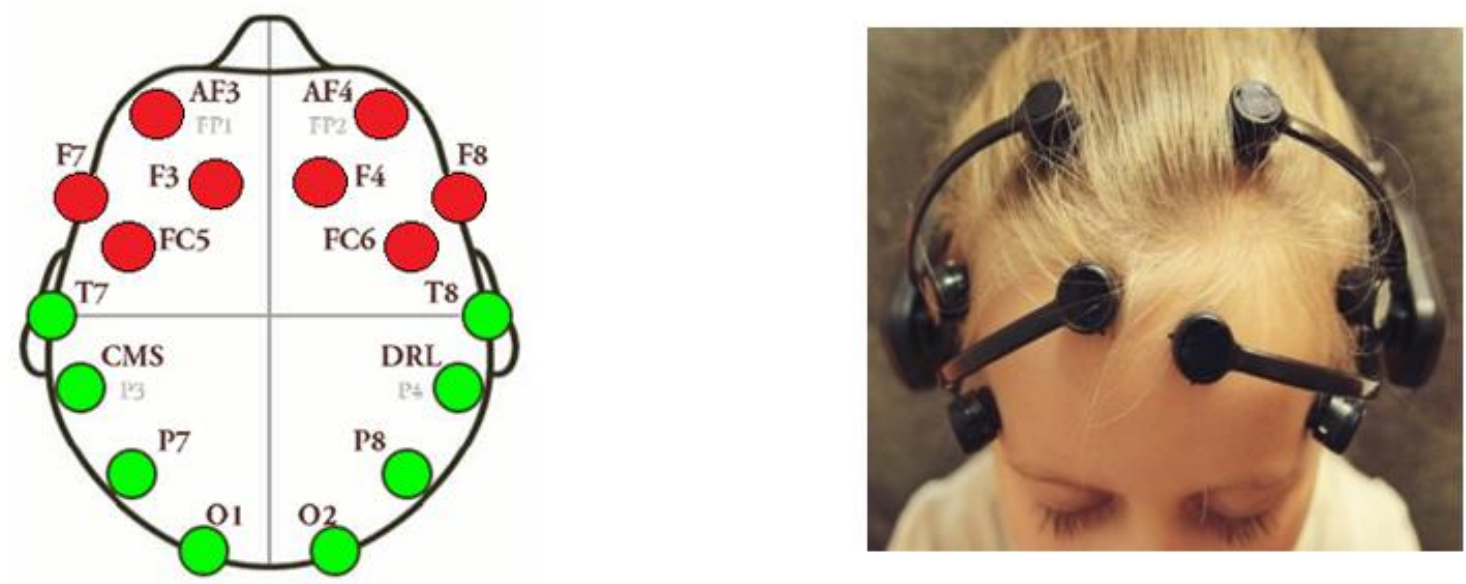

Figure 25. The location of the electrodes used in the experiment

The combination of simultaneously present rhythms forms a specific spatial-frequency EEG pattern. Patterns are typical for different types of cognitive activity and are highly individually specific. The ability of an individual to establish rhythmic EEG patterns when performing certain cognitive tasks called "encephalographic" portrait of personality [29].

During the experiment an EEG signal was recorded with a sampling frequency of $128 \mathrm{~Hz}$ for various emotional states: calm (neutral), positive emotions and negative emotions. There was no state of strong emotional arousal. The source signal of each sensor is decomposed into frequency rhythms using a discrete Fourier transform. Figure 26 shows a graph of the average spectral power of the frequency bands in 6 secs for the AF3 sensor in various emotional states.

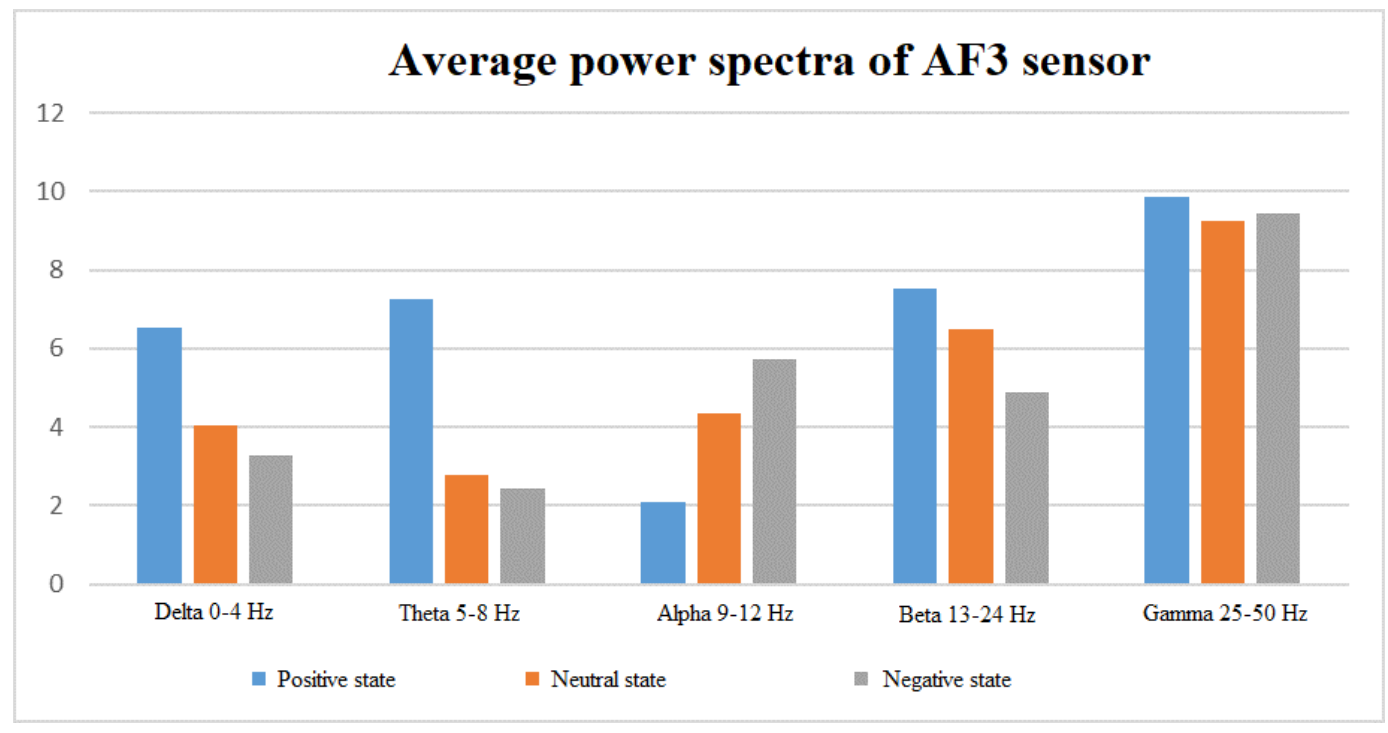

Figure 26. Average power spectra of AF3 sensor

Determining the sign of an emotion is a classic classification task. To solve it, it is necessary to determine the sign of the emotion at a specific point in time by analyzing the EEG signal. If we consider the state of rest as 0 , positive emotion as +1 , negative as -1 , the definition of the sign of the emotion can be considered as a deviation from the neutral state.

A number of studies shows [34] that in determining the sign of an emotional reaction, it is necessary to rely on changes in the power of the alpha rhythm and beta rhythm in the frontal and temporal lobes. With positive emotions, there is a strong depression of the alpha rhythm in comparison with the neutral condition, as well as an increase in the power of the beta rhythm (see tab. 1). 
Table 1. Frequency rhythms with different emotional signs

\begin{tabular}{|l|l|l|}
\hline $\begin{array}{l}\text { Frequency } \\
\text { rhythm }\end{array}$ & Positive emotions & Negative emotions \\
\hline Alpha rhythm & $\begin{array}{l}\text { More pronounced depression of the alpha } \\
\text { rhythm in comparison with the neutral } \\
\text { condition in the frontal and temporal } \\
\text { lobes. }\end{array}$ & $\begin{array}{l}\text { The power of the alpha rhythm is greater } \\
\text { than or equal to the power of the alpha } \\
\text { rhythm with a neutral condition in the } \\
\text { frontal and temporal lobes. }\end{array}$ \\
\hline Beta rhythm & $\begin{array}{l}\text { Increase of beta rhythm power compared } \\
\text { to neutral condition in frontal and tem- } \\
\text { poral leads. }\end{array}$ & $\begin{array}{l}\text { Decrease in amplitude in comparison } \\
\text { with a neutral condition in frontal assign- } \\
\text { ments. }\end{array}$ \\
\hline Theta rhythm & $\begin{array}{l}\text { The change in the power of theta rhythm } \\
\text { parison with the neutral condition de- } \\
\text { pending on the gender. }\end{array}$ & $\begin{array}{l}\text { The change in the power of the theta } \\
\text { rhythm in the frontal and temporal leads } \\
\text { in comparison with the neutral condition } \\
\text { depending on the gender. }\end{array}$ \\
\hline
\end{tabular}

The coefficients are placed in accordance with the significance of the spectral power, they also reflecting the weight of the frequency rhythm to determine the sign of emotional activity.

\section{Description of the software platform}

As a software platform for processing the EEG signal, the Python programming language version 3.7 was chosen. The NumPy package was chosen as a library for mathematical operations. NumPy is a fundamental package for scientific computing in Python, providing:

- powerful N-dimensional array object;

- complex (broadcast) functions;

- tools for integrating $\mathrm{C} / \mathrm{C}++$ and Fortran code;

- algorithms linear algebra, Fourier transform and extended possibilities of random numbers.

In addition to obvious scientific applications, NumPy can also be used as an effective multidimensional container of common data. The ability to define arbitrary data types allows you to easily and quickly integrate with a wide range of databases.

\subsection{Using the deep machine learning in the task of classifying an emotional sign}

TensorFlow was used as a library for machine learning. It is a neural network that learns how to solve problems by positive amplification and processes data at various levels (nodes), which helps to find the desired correct result with a given level of the training signal approximation error. This kind of machine learning is very well adapted for research purposes. The library was developed by the Google Brain team for a variety of tasks, including searching for images and improving speech recognition algorithms. As a starting point for the use of machine learning technologies (and later - soft computing), for recognizing emotions through the EEG signal, the TensorFlow library makes it easy to integrate into the applications self-learning elements and functions of artificial intelligence designed for speech recognition, computer vision or natural language processing.

The principle of working with TensorFlow involves the compilation of a graph of operations, data transfer and the work of calculations. The graphs allow define the calculation process, where the vertices perform operations, and the edges describe the connection between them. Thus, when adding two numbers, it is necessary to define a vertex with two inputs (numbers for addition), some calculations (addition function of two numbers) and an output (result).

Deep learning is a subset of machine learning. Usually, when people use the term deep learning, they are 
referring to deep artificial neural networks, and somewhat less frequently to deep reinforcement learning. Deep artificial neural networks are a set of algorithms that have set new records in accuracy for many important problems, such as image recognition, sound recognition, recommender systems, natural language processing etc. We are using it to identify the sign of emotion.

It was decided to implement a classifier based on a convolutional neural network in order to be able to assign features in the original data set. This feature is especially useful in the problem under study, since it is practically impossible to select significant features in the initial data set in manual mode in accordance with desired output. This significantly limits the possibility of using other types of classifiers. The training of the classification algorithm for EEG signals was based on data from four channels AF3, T7, F71 and F8. As an activation function, a rectified linear unit, specified by the expression, was used:

$$
f(x)=\max (0, x),
$$

where $x$ is the input to a neuron.

In order for TensorFlow to train the model, it is necessary to set the loss function. As the loss function cross entropy was used. Cross entropy is extremely important for modern systems, because it makes it possible to create highly accurate forecasts, even for alternative indicators. Into the learning algorithm, the power values of the spectra are fed to the input for each of the frequencies (alpha, beta, gamma, theta, delta). The task of recognizing an emotion is the task of classification, so the loss function will return:

- Neutral state -0 ;

- Negative state - - 1;

- Positive state -+1 .

Figures 27 and 28 show the visualization of data to the input of the neural network.

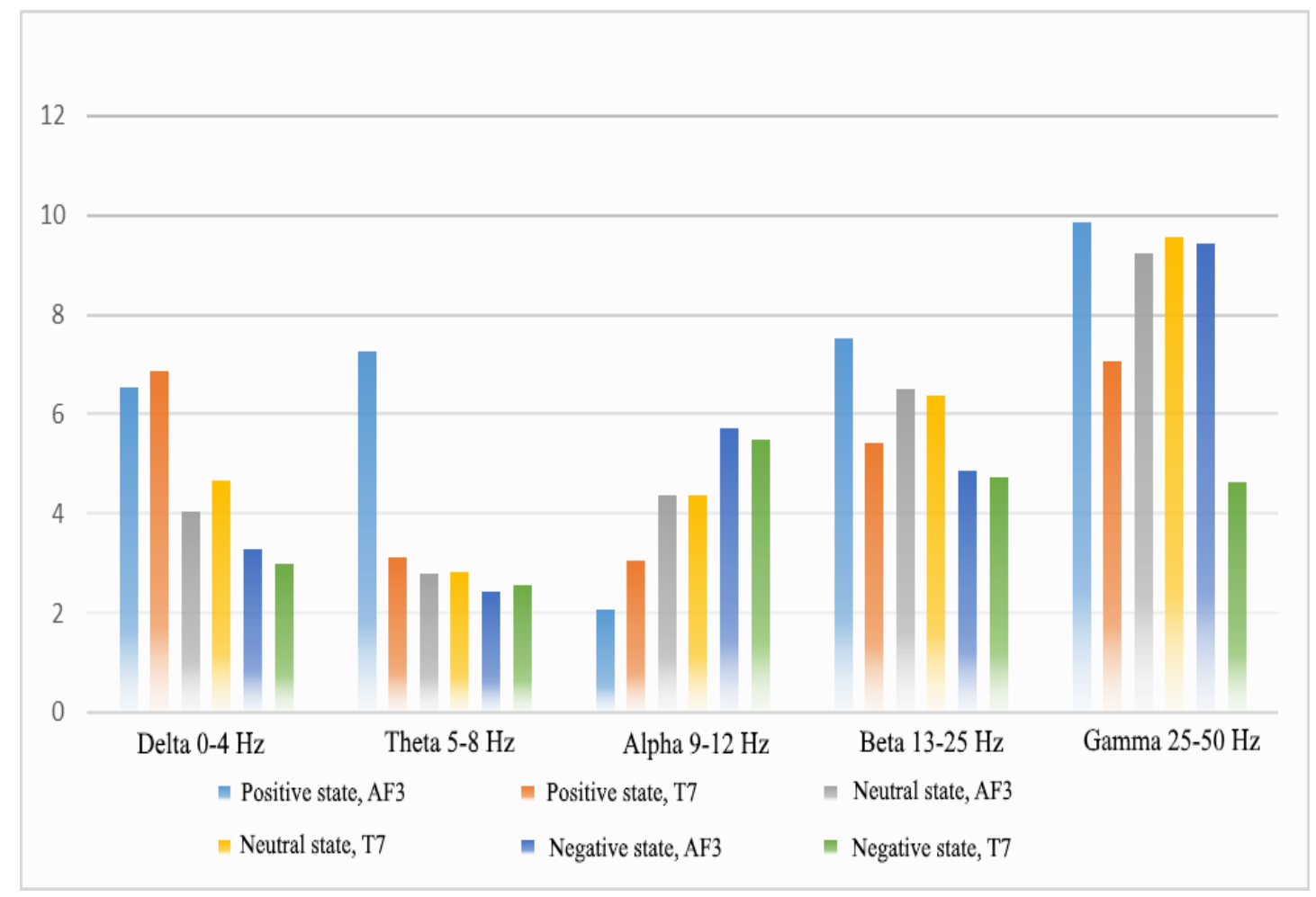

Figure 27. AF3 and T7 sensor power spectra for positive, negative emotions and a calm state 


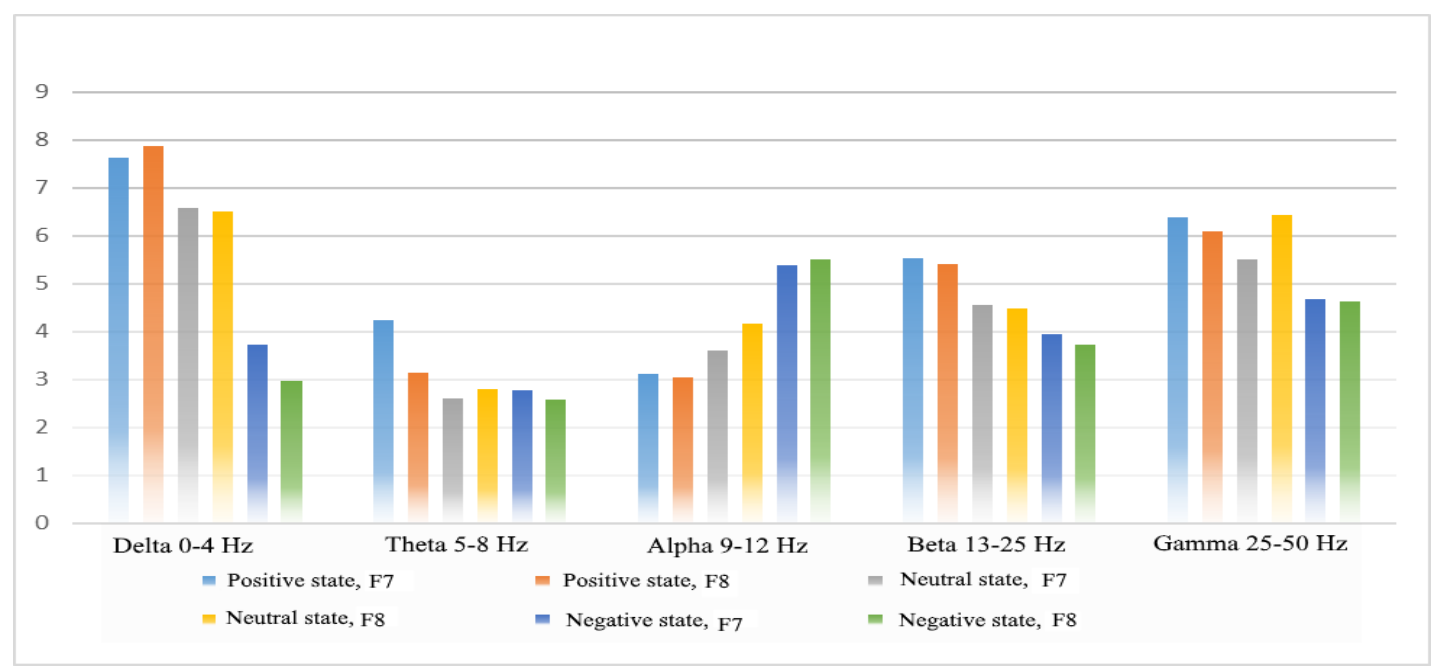

Figure 28. Power ratings of the F7 and F8 sensor spectra for positive, negative emotions and a calm state

Figure 27 reflects the total tonic activity of the spectra of emotions of a different sign for sensors located in the same hemisphere of the brain, and Figure 28 show sensors symmetrically located on opposite points of the two hemispheres of the brain.

\subsection{Soft Computing Optimizer}

Figure 27 shows the result of the neural network: the coefficient of deviation from the neutral state, obtained after processing the EEG signal, decomposed into frequency bands.

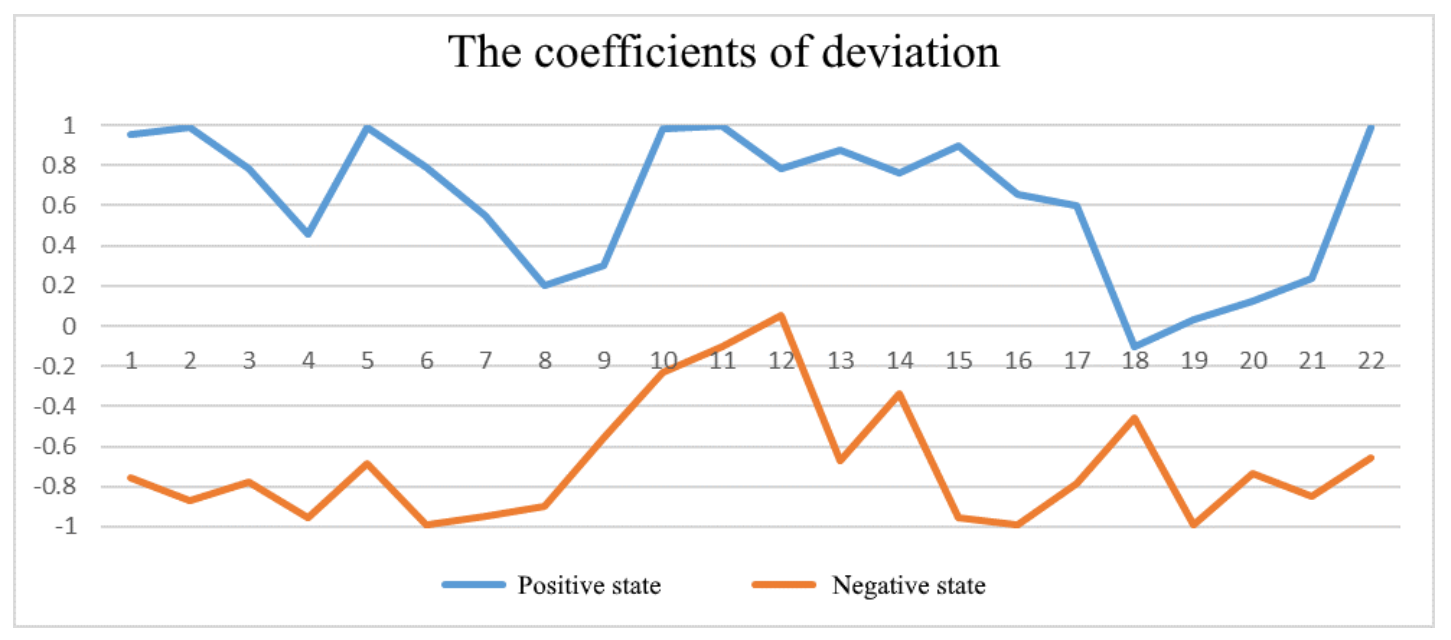

Figure 29. The coefficients of deviation from the neutral state of emotions of a different sign

As a part of the task, machine learning is used to determine the sign of the emotion at a particular point in time. For a correct description of the general psychophysical state of the operator, it is necessary to use the soft computing optimizer. Since emotions are characterized by clearly pronounced intensity, limited duration, awareness of the reasons for its appearance; connection with a specific object, circumstance; polarity, an approximation of the coefficient of deviation from the neutral state is necessary.

Remark. SCOptimizer software is used to create sophisticated knowledge bases. Soft Computing Optimizer of knowledge base (SCOptKB ${ }^{\mathrm{TM}}$ ) is a software toolkit for creating automatic fuzzy models and solves the universal approximator design problem of ill-defined control objects. The SCO uses sets of values of the input-output vector to create and optimize a fuzzy model. To perform various optimization algorithms, a learning signal is needed, which represents samples of input values and corresponding output values. Training signal files can be created using the SCO or taken from other sources. Text files are processed based on regional settings that define characters for the decimal point, the thousands separator, and so on. The default values for these parameters are set in Windows. If the settings do not match the signal format, they can be changed at any time. After the change, the parameters are saved in the model and will be used for further data processing. Regional settings affect the reading and writing of text data and model files. 
The first step of model optimization is the definition of shape of membership functions of fuzzy sets of input and (if used by the model) of output variables. SCOptimizer supports two modes of MF's shape definition: using uniform distribution method or with GA1 optimization algorithm.

Uniform distribution method distributes fuzzy sets on signal change interval according to signal probability distribution and user selected shape of membership functions.

GA1 algorithm tries to find best possible combination of number of fuzzy sets per variable, membership function shape and overlap coefficient between neighbor fuzzy sets. For each combination it performs uniform distribution algorithm and tries to maximize the mutual possibility of the fuzzy sets of each variable.

The main part of the model is a rule database. It stores data, which shows which output should be activated for given input. SCOptimizer supports two types of rule database: complete database and LBRW database.

Rules of complete database present all possible combinations of fuzzy sets of input variables. Number of rules in complete database equals to product of numbers of fuzzy sets of input variables. This will result in extremely large database and very slow optimization speed if you will try to use it with more than one-two variables. LBRW database store not all the rules, but only a number of rules selected with "Let the Best Rule Win" algorithm. LBRW algorithm selects those rules, which contribute the most noticeable part of the output. Reducing number of rules with LBRW algorithm provides faster optimization speed without loss of model precision.

After the database was created it should be filled with actual rule data. This is accomplished on the final step of model creation - rule database optimization. SCOptimizer uses genetic optimization algorithm (GA2) to tune database parameters.

Quality of the model created during previous steps may still be inadequate. In order to improve model quality GA3 algorithm is used. It alters shapes of membership functions and optimizes model output with fixed number of membership functions and database structure. Error back propagation algorithm can be used to improve model output but fine-tuning database parameters using classical gradient optimization method.

SCOptKB ${ }^{\mathrm{TM}}$ supports model export in a $\mathrm{C}$ program. The code in these files is written with minimal use of functions from the standard $\mathrm{C}$ language library and can be compiled by any $\mathrm{C}$ compiler, including those oriented to embedded systems and microcontrollers [8]. To approximate the training signal, the knowledge optimizer is used with the selected model of fuzzy inference (Sugeno 0 order). The coefficient of deviation from the neutral state and the identification value of the corresponding emotion are used. At the next stage of designing a knowledge base for fuzzy inference, a full knowledge base is automatically generated, and the right parts of the rules are further optimized (see Fig. 30).

The first layer is a layer of input variables: the spectral density of the signal power and expert evaluation. The second layer is fuzzy term-sets of input variables. The third layer corresponds to the rules of the knowledge base with the corresponding rule number in the rule base. The last layer is the output layer, which displays the numeric parameters of the rule.

The optimizer of knowledge bases on soft computing automatically forms the optimal structure of the neural network, allows from the point of view of computational mathematics to approximate the training signal with the required (given) approximation error, and from the point of view of the theory of artificial intelligence implements a deep machine learning algorithm.

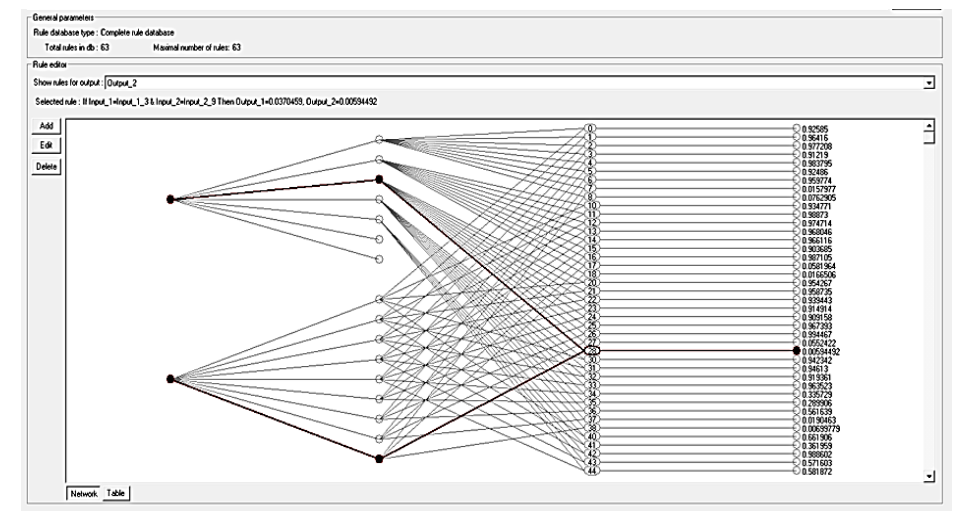

Figure 30. The rule base is in the form of a network with four layers 
In Figure 31, the first graph shows the training signal and the model of the output variable.

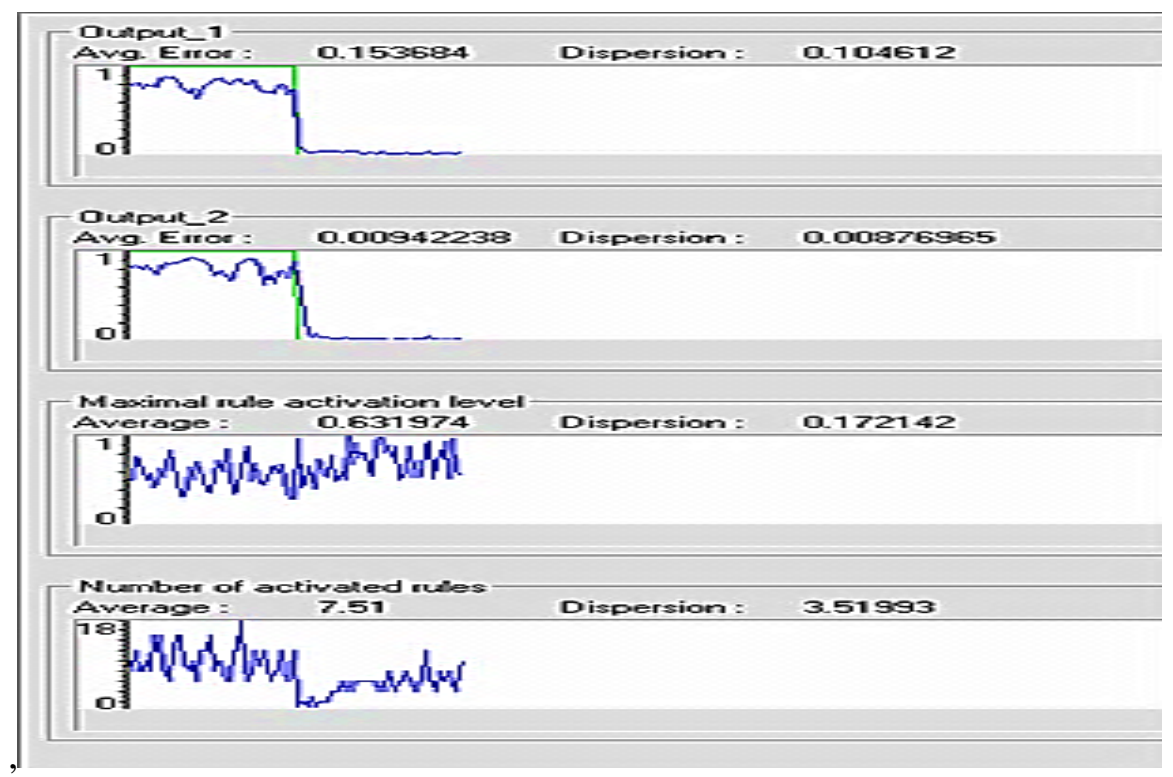

Figure 31. The result of the model is the output variable

The green line displays the training signal and the blue line shows the model output. On the second and third graphs presented the maximum level of activations of the rules and the number of activated rules.

\section{Examples of solutions}

In 1995 the robotic unicycle [35-37] and in 1994 robot for service use [11] was developed with the biomechanical mechanism description of emotion, instinct and intuition as corresponding look-up tables based on expert estimation of finite number of production rules and linguistic variables with fuzzy logic inference. In addition to design of look-up table in [35] in the structure of intelligent control system (for the feeling support of comfort car passenger) "friendly ship" bio-inspired frequency filter was introduced; robust control of passenger comfort feelings based on quantum soft computing technology is achieved [36]. In this article applied SCOptKB ${ }^{\mathrm{TM}}$ toolkit [37] extract information from EEG signal, design optimal structure of fuzzy neural network and create the universal approximator of deep machine learning processes with optimal finite number of production rules, choice optimal type and parameters of linguistic variables for fixed model of fuzzy logic inference. The learning architecture and the associated unsupervised learning algorithm of recurrent quantum neural network [38] have been modified to take into account the complex nature of EEG signal. The basic approach is to ensure that the statistical behavior of input signal is properly transferred to the wave packet associated with the response of quantum dynamics of the network. EEG signals can be considered a realization of a random or stochastic process. When an accurate description of the system is unavailable, a stochastic filter [39] can be designed on the basis of probabilistic measures cooperated with fuzzy modeling. This approach for Social Robotics design with successful emotion state recognition of ASD children and for detecting early signs of dementia [40] based on quantum deep machine learning with smart quantum computational intelligence toolkit [42] can be applied.

\subsection{Cognitive intelligent control in navigation of autonomous robot}

Usually, a regulator is installed at the facility as a control system, which, depending on the mental commands of the operator, generates a control action for the actuators. Such a controller can be a simple relay controller, where the same control actions are generated for a finite set of output commands (forward, backward, left, right). In this work, we tested a proportional controller, a proportional-integral (PI) controller with a fuzzy output unit, and a proportional-integral dirivative (PID) controller with various gain factors.

For the experiment was been select the object of control - mobile robot in the form of three-wheel vehicle with Bluetooth-control showed on Figure 32. The control device as a control processor used the Arduino Uno. Together with the engine driver- Pololu Dual MC33926. 2 (micromotor) Motor- DC 9V Motor Bluetooth module- HC-05. Power supply serves $33.7 \mathrm{~V}$ Li-On battery. 


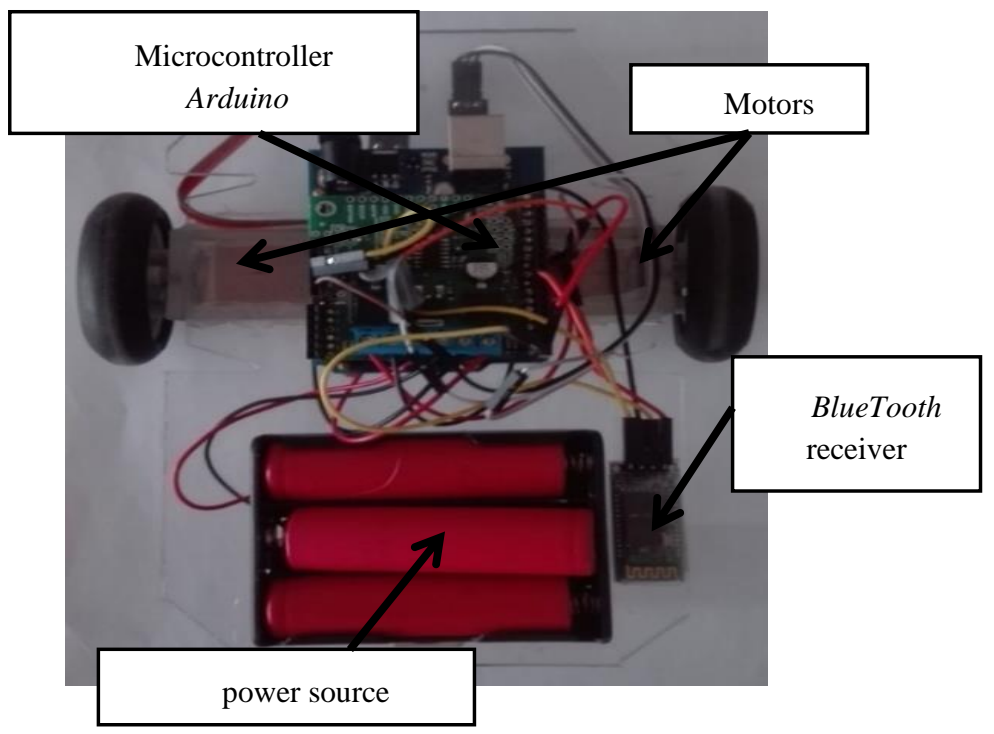

Figure 32. Control Unit

The first and easiest implementation regulator for vehicle is a proportional controller. Such a regulator sends a proportional value of motors cars depending on which team has the greatest affinity to recorded in advance mental command (see Figs 33 and 34).

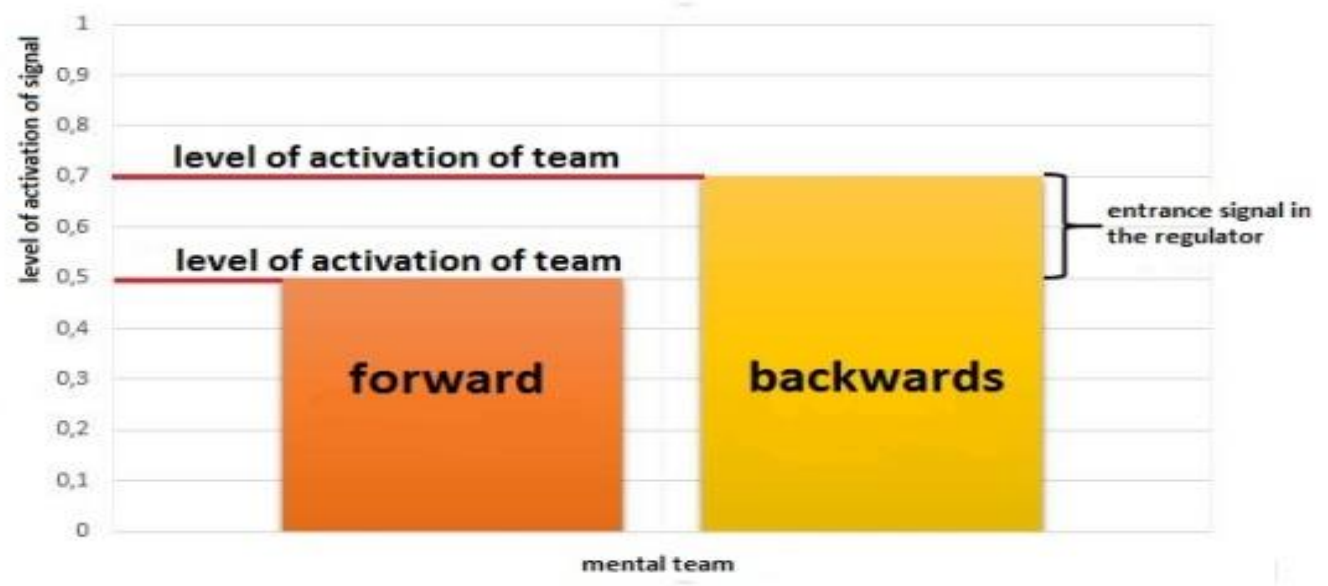

Figure 33. Activating the commands in the proportional controller

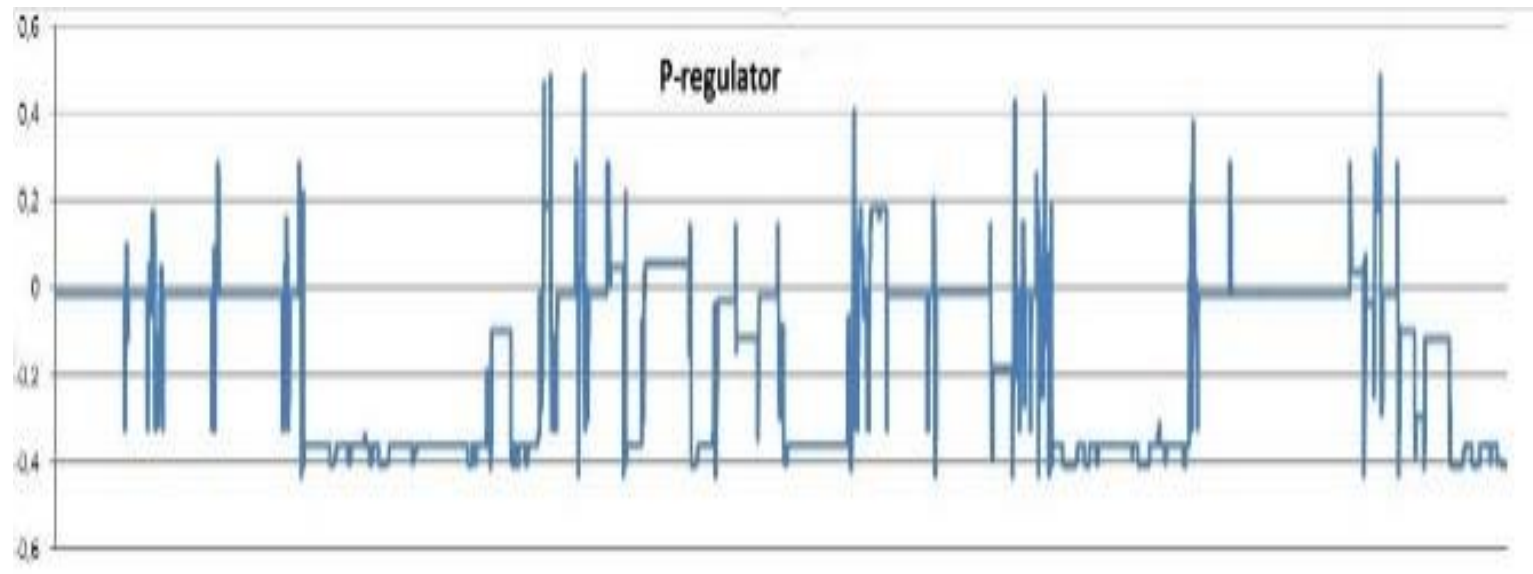

Figure 34. Control impact produced p-controller while moving back and forth

For example, activation command associated in pairs of movement forward and backward was made according to the difference of activation levels for these commands. 
Next, let us look at the process of design PI regulator using SCO. To do this, in the first phase the expert generates a training signal, driving based on proportional regulator machine. During system operation, recording the signals received from the block recognizer. Coming from this signal by adding formed integral component (see Fig. 35). Then the expert put the respective control impact based on previous experience with the system.

To approximate the teaching signal (see Fig. 36) applied the developed SCO with selected the model of fuzzy inference (Sugeno type models). As teaching signal used the signal from the block signal recognition EPOC, as well as the integral value of the signal.
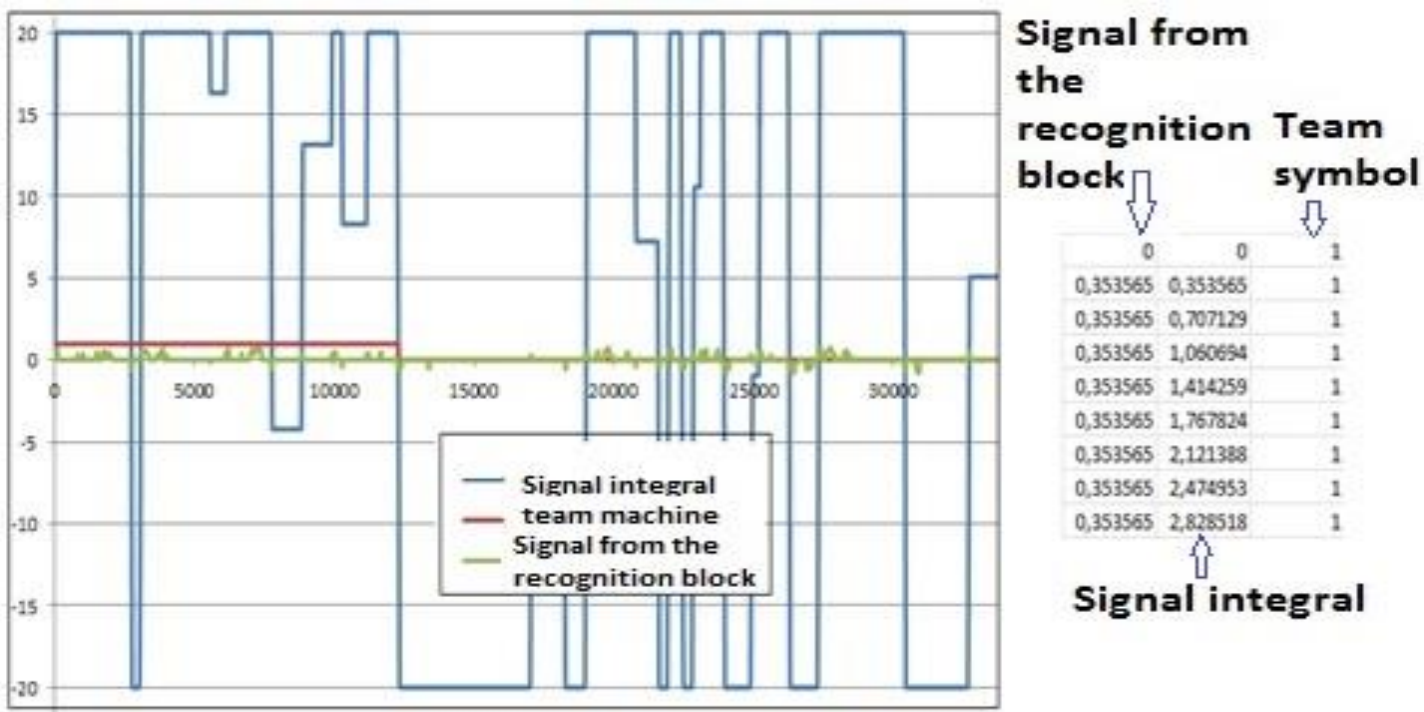

Figure 35. The training signal

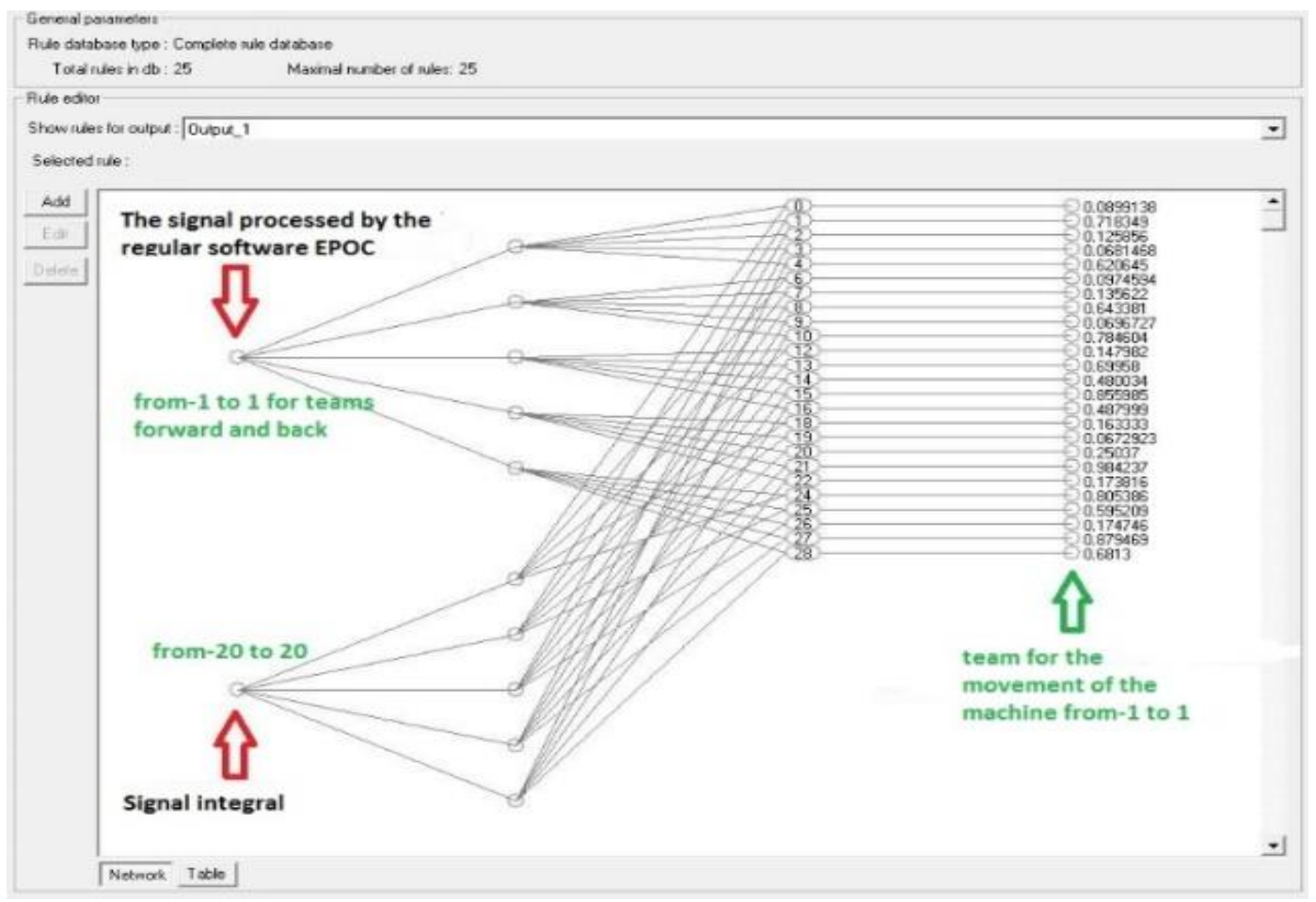

Figure 36. Neural network fuzzy inference

At the next stage of design for fuzzy knowledge base withdrawal is carried out in automatic mode formation full knowledge base and further optimization of right-hand sides (see Fig.37). 


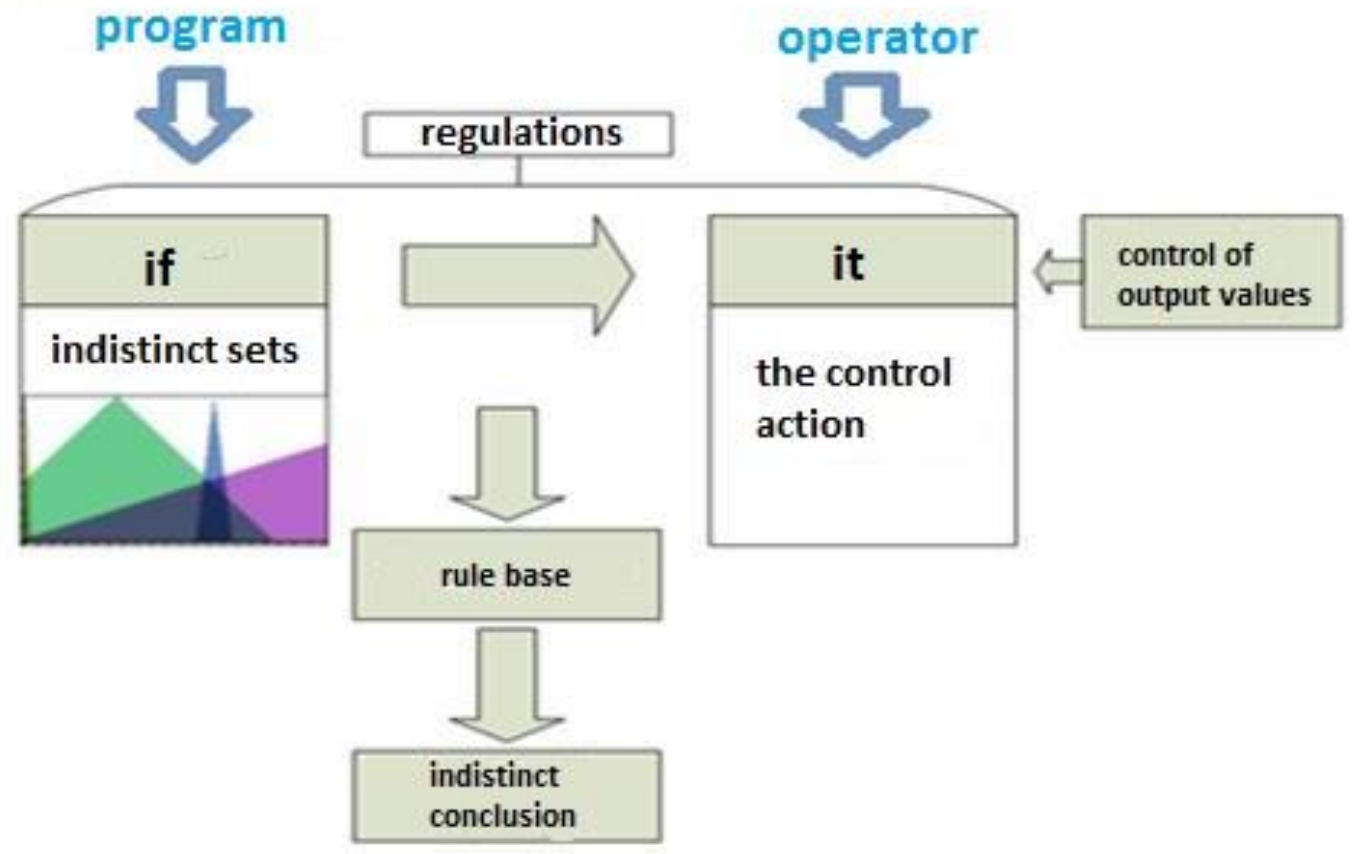

Figure 37. Block diagram the formation rules in the knowledge base

In other words, at the entrance to the neural net receives commands from the software module signal recognition (forward, backward, left and right) the output value is the commands then receives vehicle.

The knowledge base is applied in conjunction with PI controller. Using soft computing need to build more "soft" structure to control. For this purpose, created linguistic variables (LV) for each of the commands recorded in the system, was formed a complete knowledge base (see Fig. 38). The right side of the regulator contains appropriate values for control action using PI controller. Thus, the activation level of rules in base corresponds to the activation level of the control action.

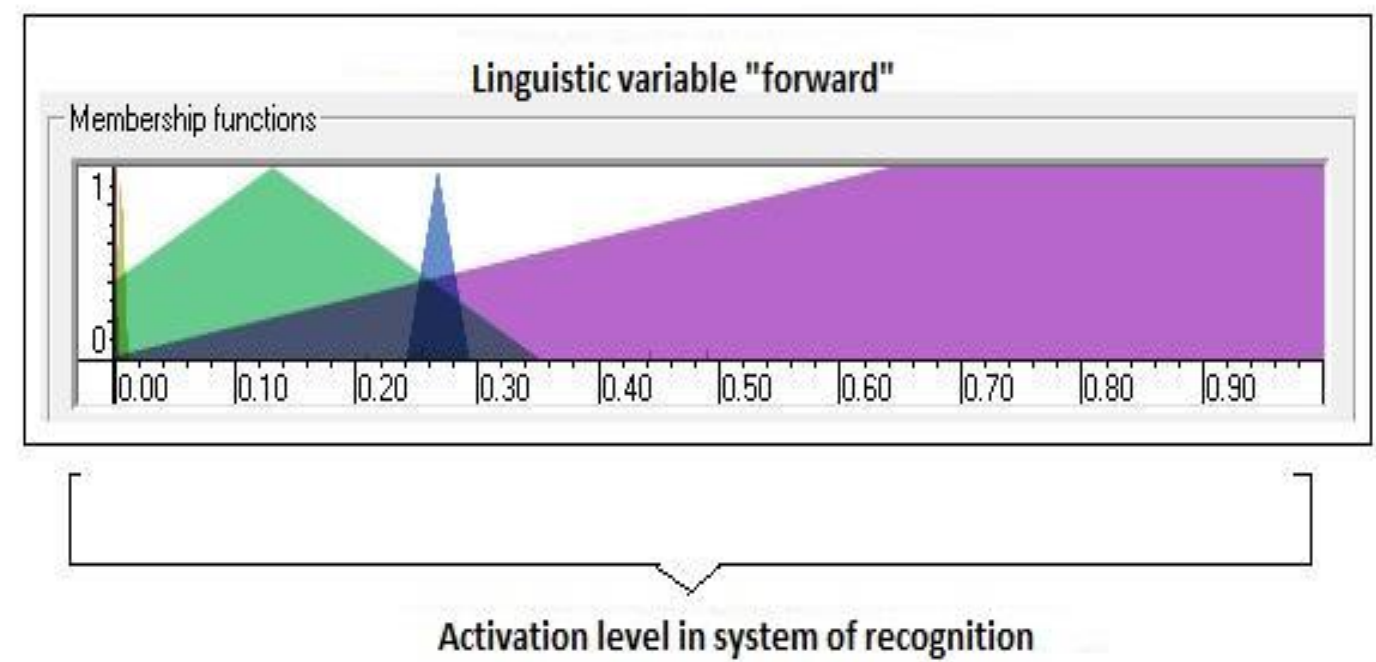

Figure 38. An example of the linguistic variable for the team forward

Figure 39 shows the result of cognitive motion control of mobile robot in maze based on the PI-regulator. 


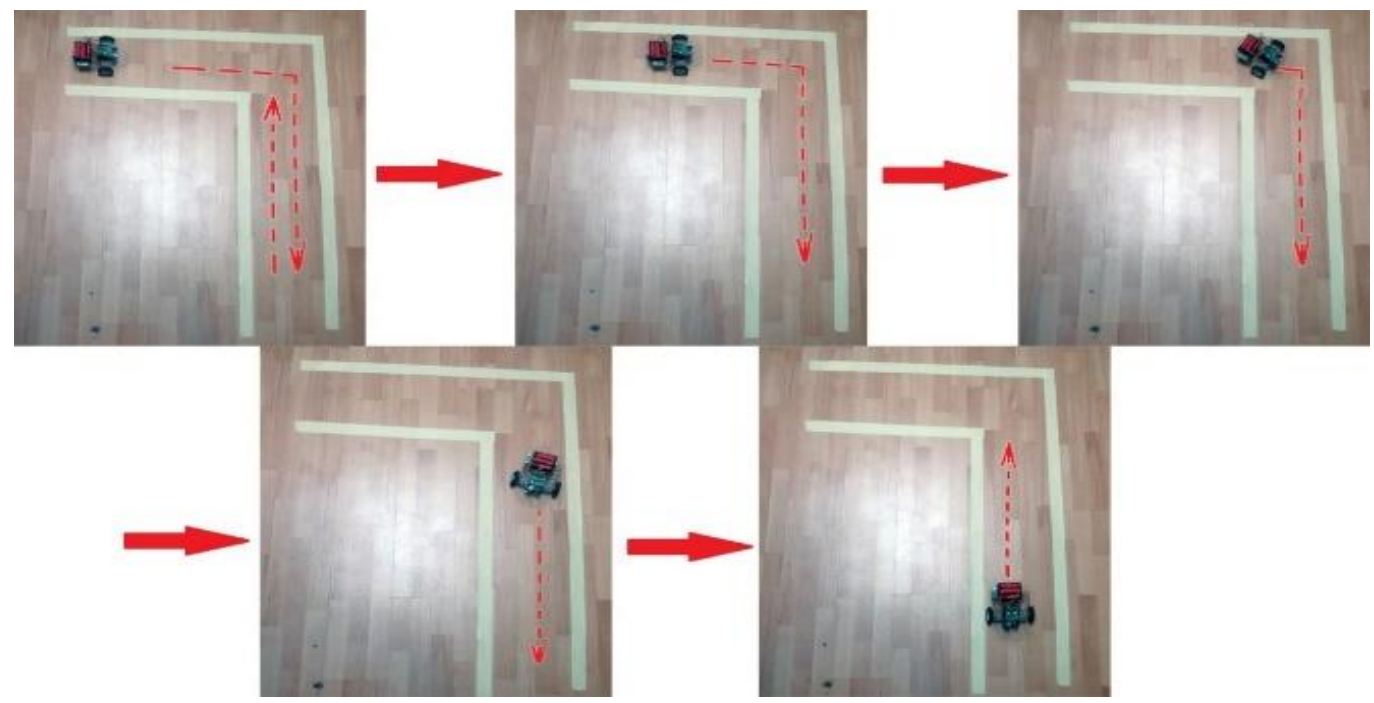

Figure 39. Controlling the trajectory of mobile robot based on the PI-regulator

The following verified regulator to control machines was PID controller with constant coefficients. The coefficients of the regulators were PID $1\left[\begin{array}{llll}1 & 0.1 & 1\end{array}\right]$ and for PID 2 [ $\left.\begin{array}{lll}3 & 0.1 & 10\end{array}\right]$.

In Figs 40 and 41 shown the commands of control systems to manage control object. The first chart, green introduced the target signal, which corresponds to a movement back and forth, and the rest of the colors allocated to the activation levels and PI controller with knowledge base.

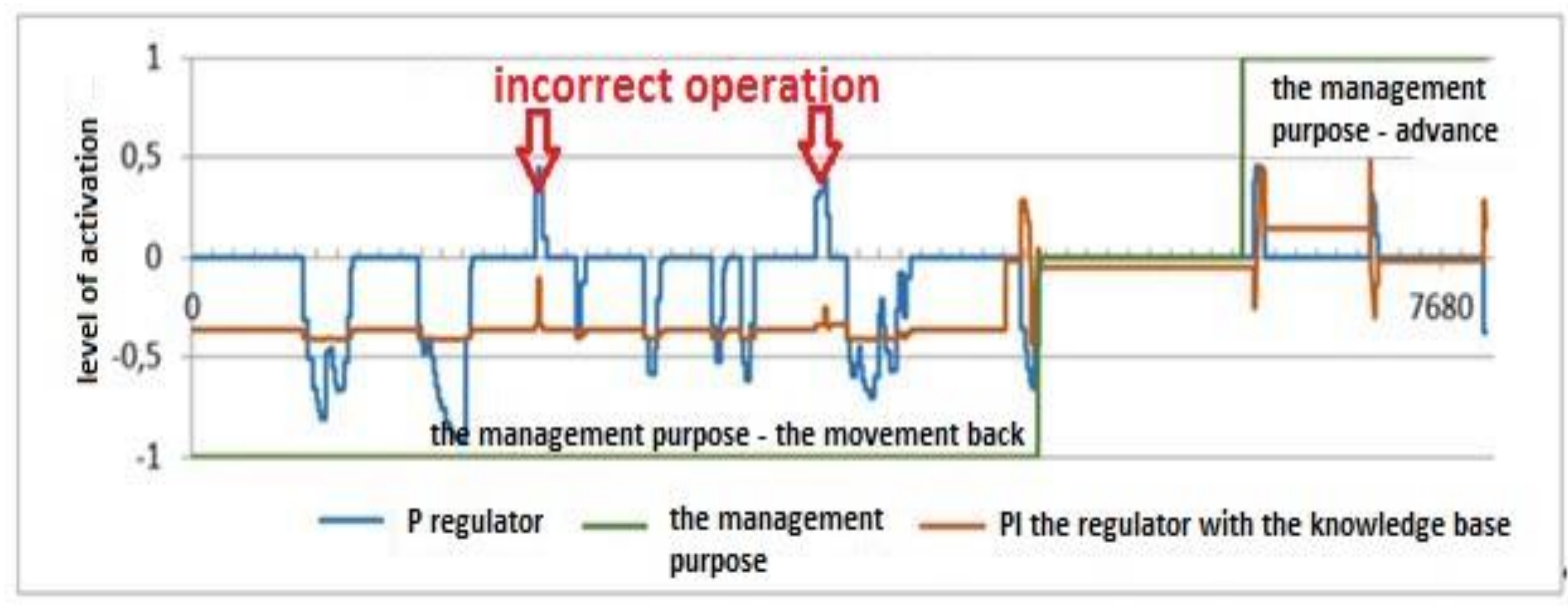

Figure 40. Controlling actions produced by standard signal and PI regulator. Forward and backward

As can be seen from the graphs in Fig. 40, when the task motion vehicle is back, and concentration occurs thinking process on that team, recognition block is not always correctly identifying and control the machine. Vehicle work in spurts or even goes to the other side (false positives), PI control compensates this, and additional add-in as an integral component in the knowledge base, allows a smooth sequence of commands and reduce errors in reaching the goal. Moreover, the system becomes adaptive and learnable, because The basis of the base is the software tool SCO.

On Fig. 41, move to right corresponds to 1, and the movement to the left corresponds to -1. 


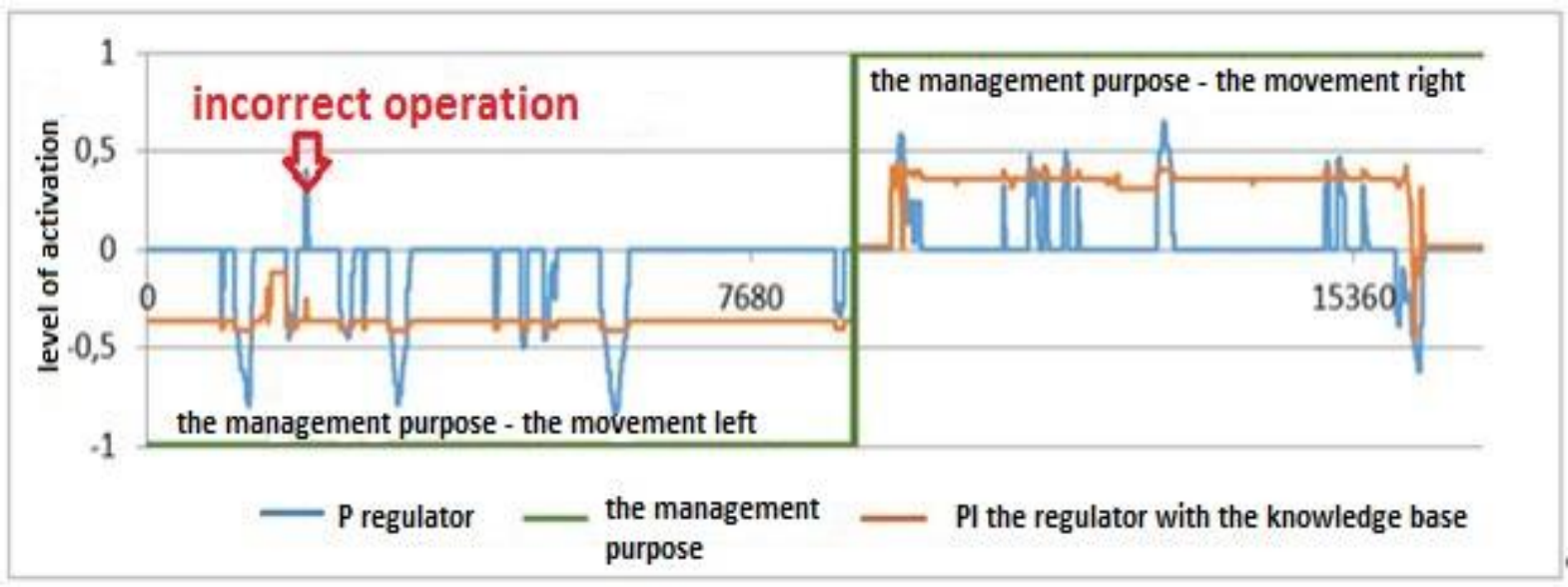

Figure 41. Control actions produced by relay and PI adjuster when moving left and right

Additionally, there was decry the problem of the motion using control system with PID regulator.
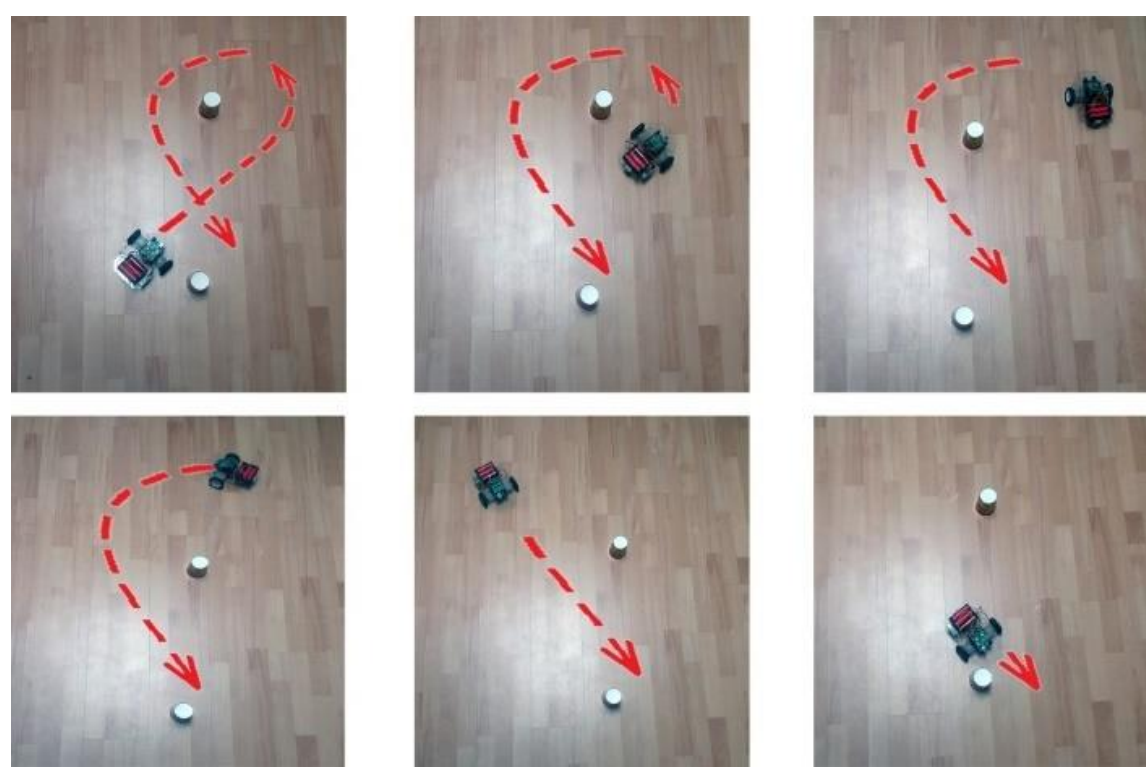

Figure 42. Detour obstacles control system with PID regulator

Figure 42 presents the results of an experiment using PID controllers with different coefficients of gain control action. The odds were set in manual mode. Differential component in PID controller associated with the speed of the operator activates the mental command.

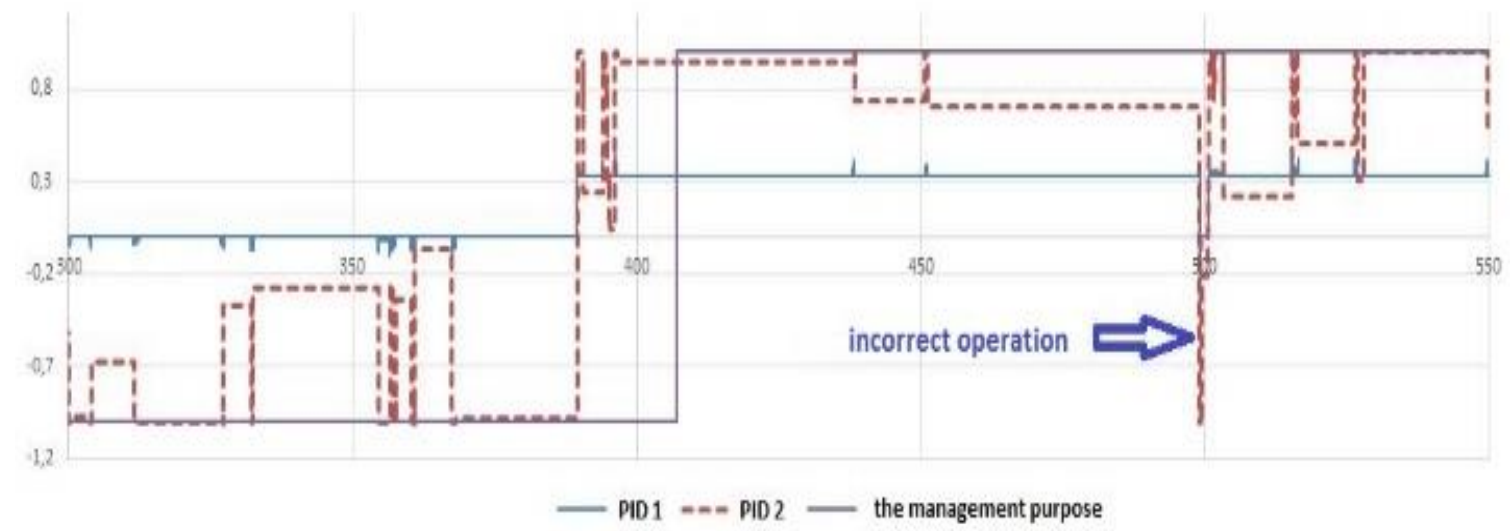

Figure 43. Control actions produced PID regulators when driving forward

The choice of gain factors influence naturally on the computation the action of controller and the operation 
of the system as a whole. However, establishing the optimal values of coefficients for each point in time is relevant and very interesting task. When incorrect (false) installation values the same way there has been an incorrect actuation, control object moves in spurts.

To compare the results obtained in the experiments used value is the mean deviation from the desired result. As can be seen from table 2 and Fig. 44, using a more complex controller, the deviation has reduced. However, the wrong setting of the gain increases the deviation of the system from the intended target.

Table 2. Compare mean deviation of different controllers

\begin{tabular}{|c|c|c|c|c|}
\hline I & $P$ & PI & PID1 & PID2 \\
\hline Mean deviation & $6^{0,84}$ & $\begin{array}{ll} & 0,85 \\
3 & \end{array}$ & 0,860 & 0,505 \\
\hline
\end{tabular}

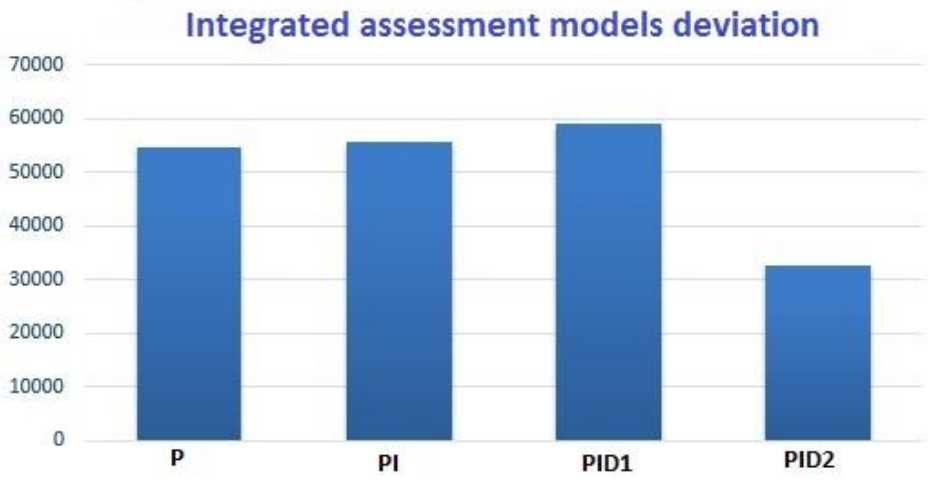

Figure 44. Cumulative score deviation module

Analysis of results of experiments showed that quality control is greatly improved when more complex control schemes.

\subsection{Robotic unicycle}

We attempted in the present work the emulation of human riding a unicycle by a robot. It is well known that the unicycle system is an inherently unstable system and both longitudinal and lateral stability control are simultaneously needed to maintain the unicycle's postural stability. It is an unstable problem in three dimensions (3D). However, a rider can achieve postural stability on a unicycle, keep the wheel speed constant and change the unicycle's posture in the yaw direction at will by using his flexible body, good sensory systems, skill and intelligent computational abilities.

Investigating this phenomenon and emulating the system by a robot, we aim to construct a biomechanical model of human motion dynamics, and also evaluate the new methods for the stability control and analysis of an unstable system. We developed a new biomechanical model with two closed link mechanism and one turntable to emulate a human riding a unicycle by a robot. This study of rider's postural stability control on a unicycle began from the observation of a human riding on a unicycle with vestibular model as intelligent biomechanical model including instinct and intuition mechanisms.

We consider the dynamic behavior of the biomechanical model from the standpoint of mechanics, decision-making process, action logic, and information processing with distributed knowledge base levels. The physical and mathematical background for the description of the biomechanical model is introduced. In this paper a thermodynamic approach is used for the investigation of an optimal control process and for the estimation of an artificial life of mobile robots [36, 37].

A new physical measure (the minimum entropy production) for the description of the intelligent dynamic behavior and thermodynamic stability condition of a biomechanical model with an AI control system for the robot unicycle is introduced. This measure is used as a fitness function in a GA for the computer simulation of the intuition mechanism as a global searching measure for the decision-making process to ensure optimal control of the global stability on the robot unicycle throughout the full space of possible solutions. The simulation of an instinct mechanism based on FNN is considered as a local active adaptation process with the minimum entropy production in the learning process of the vestibular system by teaching the control signal 
accordingly to the model representation results of [35]. Computer simulations in this study are carried out by the usage of thermodynamic equations for the motion of the robot unicycle. Entropy production and entropy measures for the robot unicycle motion and the control system are calculated directly from the proposed thermodynamic equations of motion.

Figs 45 and 46 are demonstrated the unicycle model and results of simulations.
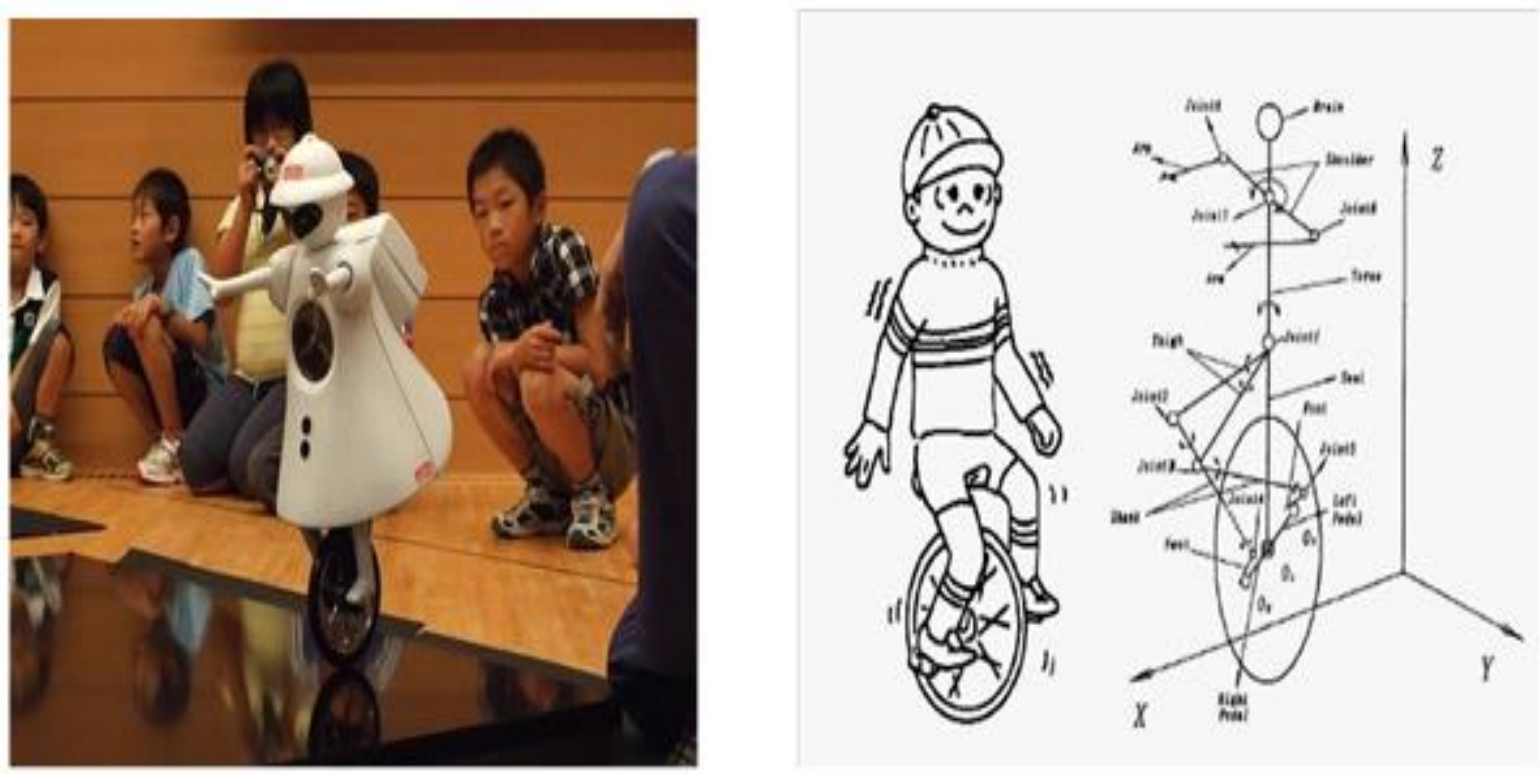

Figure 45. Robotic unicycle model

In particular, Fig. 45 shows the main idea of robotic unicycle design using Kansei and System of System Engineering approaches. With genetic algorithm the intuition of solution search is developed based on bioinspired model of unicycle rider behavior. Instinct and emotion are introduced based on fuzzy neural network and corresponding look-up tables.

From the results obtained in this study, showed at Figure 46, by the fuzzy simulation and soft computing, based on GA and FNN, it is obvious that the intelligent behavior controllability and postural stability of the robot are largely improved by two fuzzy gain schedule PD-controllers in comparison to those controlled only by a conventional PD and a fuzzy gain schedule PD-controller As a result of this investigation the look-up tables for fuzzy robust controllers of the robotic unicycle are formed with minimum production entropy in intelligent controllers and the robotic unicycle model uses this approach. The FNN controller offers a more flexible structure of controllers with a smaller torque, and the learning process produces less entropy. FNN controller gives a more flexible structure to controllers with smaller torque and the learning process produces less entropy than GA.

Thus, an instinct mechanism produces less entropy than an intuition mechanism. However, the necessary time for achieving an optimal control with the learning process on FNN (instinct) is larger than that with the global search on GA (intuition). The general approach for forming a lookup-table with GA and the fuzzy classifier system based on FNN. Intuition and instinct mechanisms are considered as global and local search mechanisms of the optimal solution domains for an intelligent behavior and can be realized by GA and FNN accordingly. For the fitness function of the GA, a new physical measure as the minimum entropy production for a description of the intelligent behavior in a biological model is introduced. 


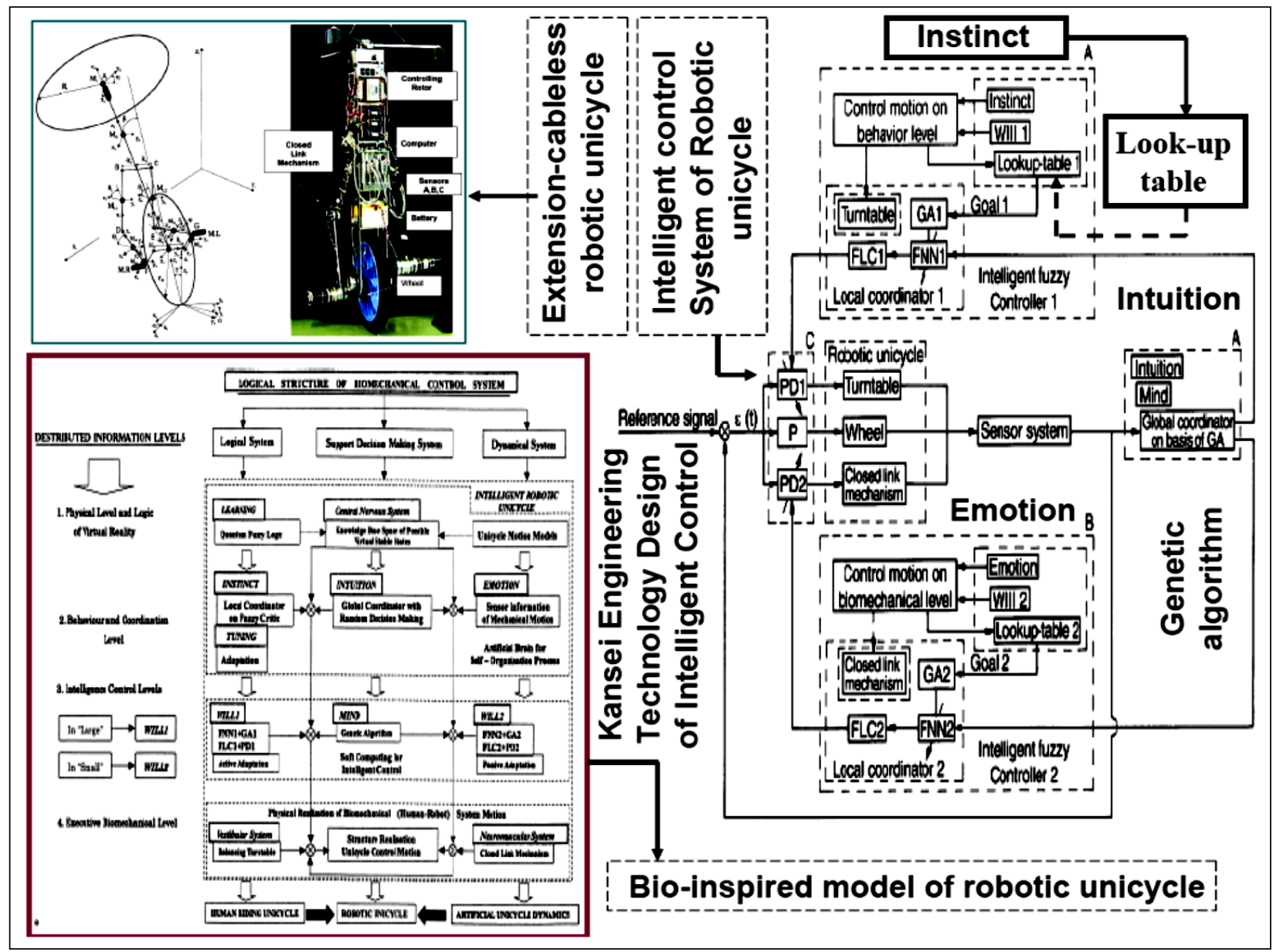

Figure 46. Simulation and experimental results

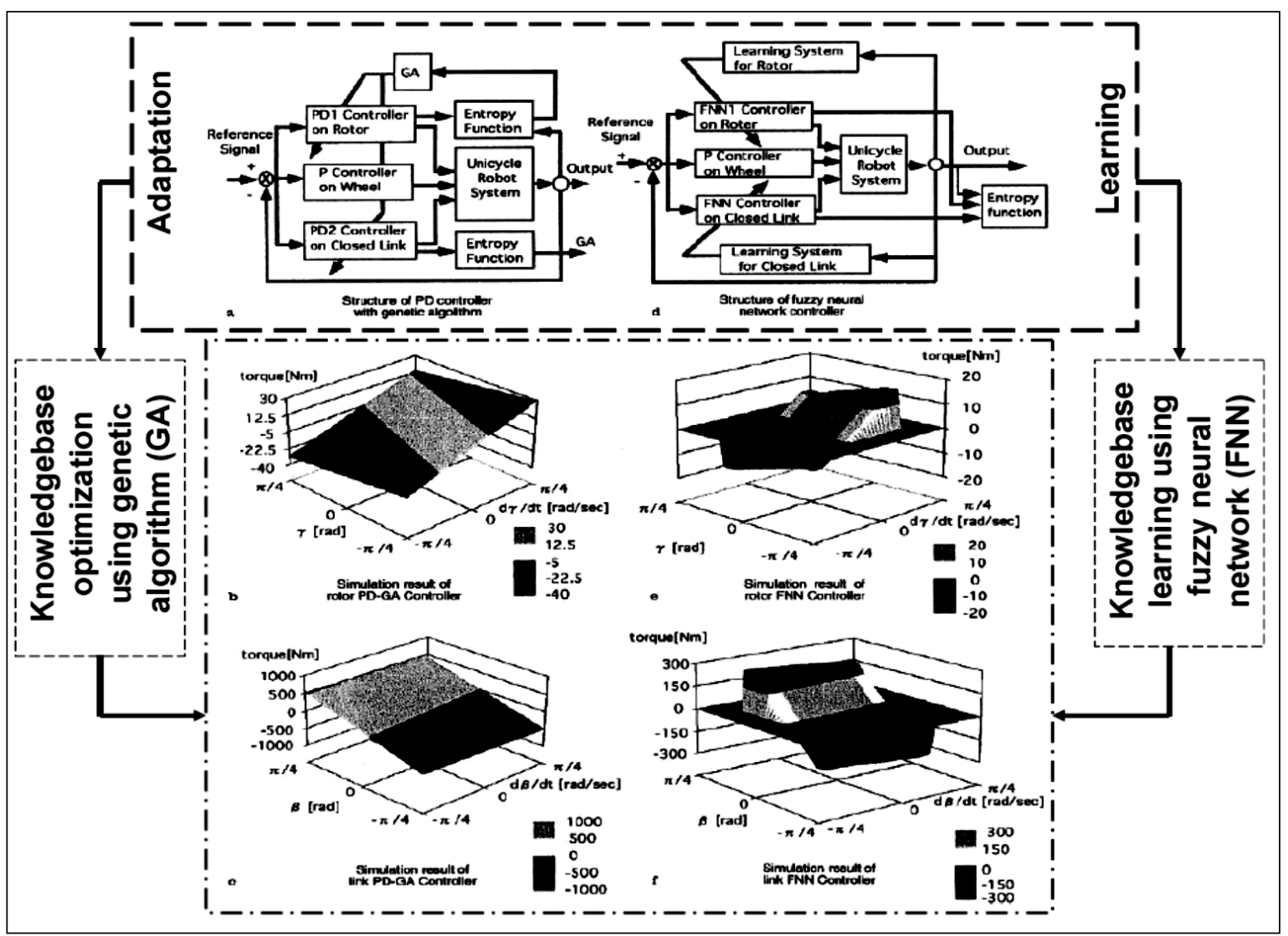

Figure 47. System simulation results of mechanics and thermodynamic behavior 


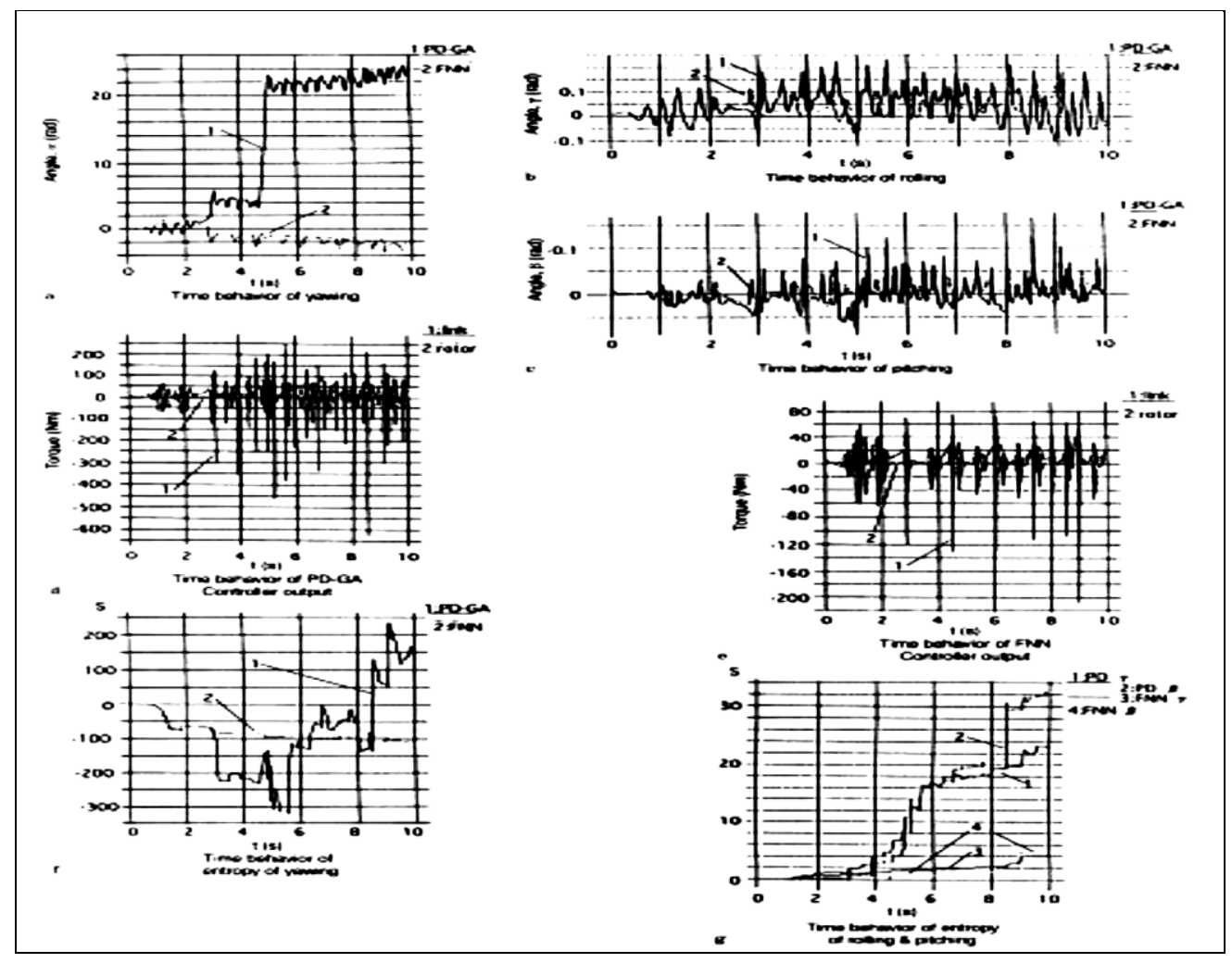

Figure 48. System simulation of robotic unicycle model

Thus, the posture stability and driving control of a human riding-type unicycle have been realized. The robot unicycle is considered as a biomechanical system using an internal world representation with a description of emotion, instinct and intuition mechanisms. We introduced intelligent control methods based on soft computing and confirmed that such an intelligent control and biological instinct as well as intuition together with a fuzzy inference is very important for emulating human behaviors or actions.

Intuition and instinct mechanisms are considered as global and local search mechanisms of the optimal solution domains for an intelligent behavior and can be realized by genetic algorithms (GA) and fuzzy neural networks (FNN) accordingly. For the fitness function of the GA, a new physical measure as the minimum entropy production for a description of the intelligent behavior in a biological model is introduced. The calculation of robustness and controllability of the robot unicycle is presented. This report provides a general measure to estimate the mechanical controllability qualitatively and quantitatively, even if any control scheme is applied.

The measure can be computed using a Lyapunov function coupled with the thermodynamic entropy change. Described above interrelation between Lyapunov function (stability condition) and entropy production of motion (controllability condition) in an internal biomechanical model is a mathematical background for the design of soft computing algorithms for the intelligent control of the robotic unicycle.

Fuzzy simulation and experimental results of a robust intelligent control motion for the robot unicycle are discussed. Robotic unicycle is a new Benchmark [25] of non-linear mechatronics and intelligent smart control. It is confirmed that the proposed fuzzy gain schedule PD-controller is very effective for the handling of the system's nonlinearity dealing with the robot's posture stability controls. Furthermore, an important result is that the minimum entropy production gives a quantitative measure concerning the controllability and also qualitative explanations.

Thus, we provide a new benchmark of Kansei engineering for the controllability of unstable nonlinear nonholonomic dynamic systems by means of intelligent tools based on a new physical concept of robust control: the minimum entropy production in control systems and in control object motion in general. 


\section{Quantum computing approach - quantum deep learning and quantum neural network}

The work carried out showed that it is possible (in principle) to classify the mental states of a human being operator, demonstrates the optimal deep machine learning ability of the system, the ability to create knowledge bases based on the recorded EEG signal and use the results to recognize emotions.

Since emotions are characterized by clearly pronounced intensity, limited duration, awareness of the reasons for its appearance; connection with a specific object, circumstance; polarity, the use of machine learning and intelligent superstructure in the form of SCO, based on fuzzy controllers, is the best tool for correctly describing the general psychophysical state of the human being operator in Affective / Kansei Engineering approach [41, 42].

The ICS robustness, obtained on the basis of such an approach, requires a minimum of initial information, both on the behavior of the control object, and on external disturbances.

An assessment of the accumulation of integral error (without using intellectual tools) can only evaluate the tonic activity of the brain, which shows a strong surge in the emotional background. Fuzzy controls allow you to slightly expand the ability to recognize the emotional background by adding production logic rules.

The combined use of an artificial neural network and soft computing optimizer on fuzzy controller allows to fully adapt the system, but it takes a long time to learn. This is critical in emergency and unforeseen situations for a system of intelligent robust control. The percentage of successful classification of the emotional sign in a human operator when working with quantum neural networks is much higher than that of classical neural networks. This leads to an increase in the reliability of the system as a whole, and allows the formation of more robust knowledge bases.

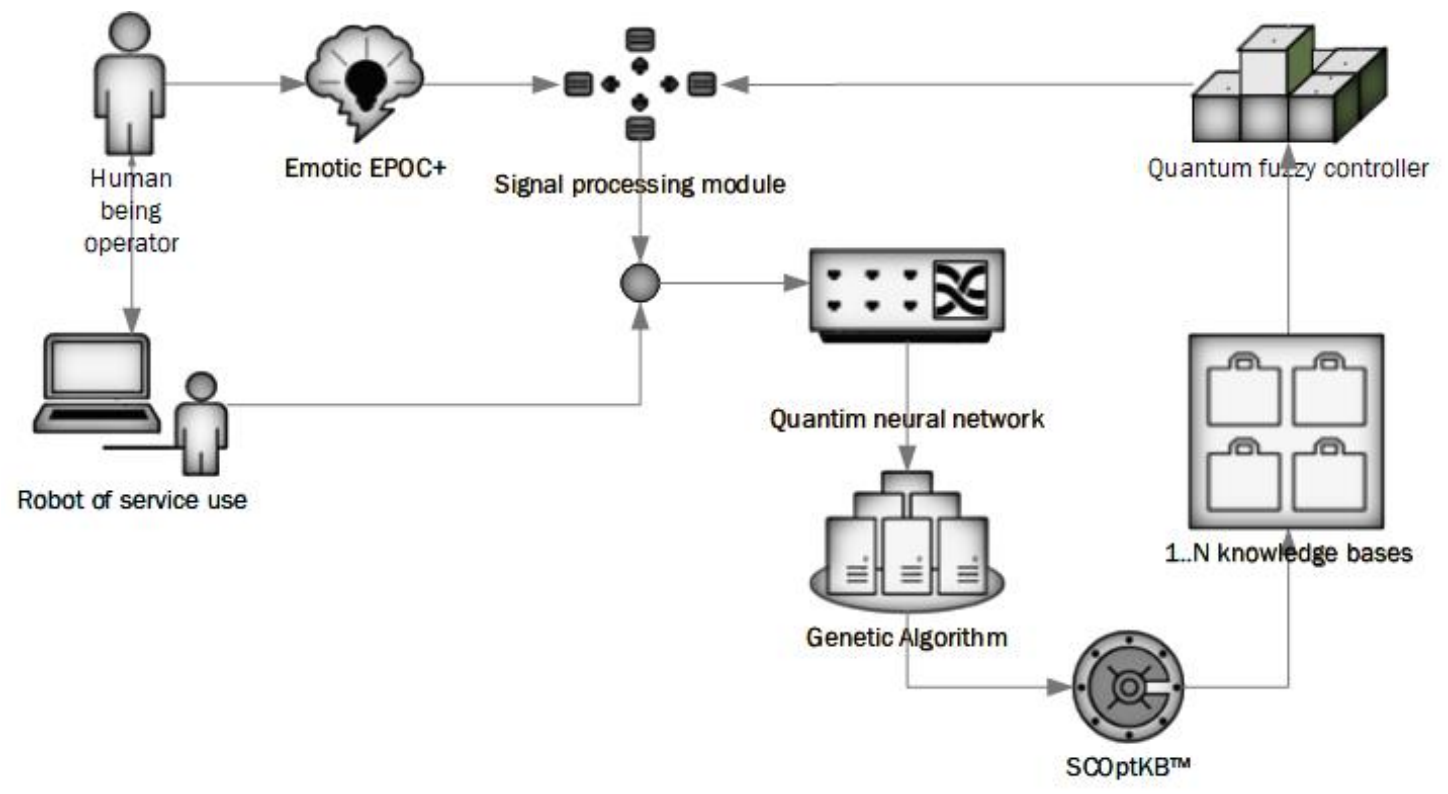

Figure 49. Intelligent control system with the integration of several fuzzy regulators

The Figure 49 shows the system with the integration of several fuzzy regulators and quantum fuzzy inference, contributing to the creation of a new quality of management: self-organization of knowledge bases online apply quantum neural network.

\subsection{Quantum neural network application}

Classic neural networks have some attractive features: parallel processing, error tolerance, the ability to learn and generalize the knowledge gained. The generalization property is understood as the ability of the neutral network to generate the correct outputs for input signals that were not taken into account during the learning. However, artificial neutral networks also face many difficulties: lack of rules for deterministic optimal architectures, limited memory capacity, time-consuming learning, etc. But there are quantum neural networks that solve these problems. 
Remark. The idea of a quantum neural network was first described in [43]. It is a combination of the concept of a conventional neural network and the paradigm of quantum computing. In 1997, A. Vlasov proposed a hypothetical model of a quantum neural network using optical interference [42]. The first systematic review of artificial quantum neural networks is given in the dissertation of T. Menner [44]. The main advantage of quantum computing over classical is quantum parallelism, which allows to work with all valid states at the same time.

There are various prototypes of quantum neural networks. Some of them are very similar to their classical counterparts, while others use quantum operators that do not have classical equivalents, for example, phase shifts. Distinguish a wide range of different structures of the quantum neural networks. It is important to note that the efficiency of using neural networks is associated with massive parallel distributed processing of information and the nonlinearity of the transformation of input vectors by neurons. In addition, quantum systems have a much more powerful quantum parallelism, expressed by the principle of superposition.

The idea of creating an artificial quantum neural network consists in replacing the classical signals arriving at the input of a neuron with quantum states with amplitude and phase. At the same time, a quantum state, depending on the linear superposition of the incoming states, should also be formed at the output of the neuron. The weights in the case of a quantum neural network (QNN) are complex numbers (which change during the training of the network (see, Fig. 50)), so that each input quantum state is not only weighted in amplitude, but also shifted in phase.

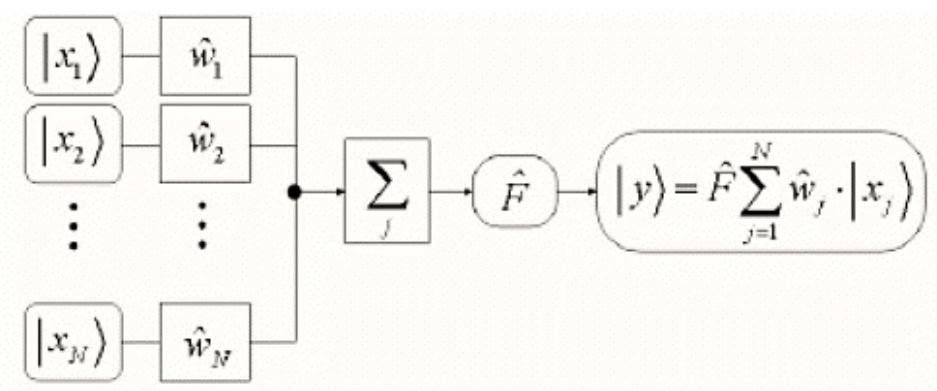

Figure 50. The mathematical model of a quantum neuron

Consider the parameterized quantum channel depicted in Fig. 51a, which describes a general feed-forward artificial QNN. The device maps inputs - a tuple of quantum and classical data - to outputs that may also contain quantum and classical parts, i.e., $(|x\rangle, \vec{x}) \mapsto(|y\rangle, \vec{y})$. Supervised training of the QNN uses inputoutput pairs as training data (e.g., the $x$ and $y=f(x)$ values from a nonlinear function) or quantum channel (e.g., a unitary quantum circuit or dissipative evolution), and attempts to optimize the QNN's parameters to make the QNN's outputs for each input match the training set. In addition to depending on the QNN architecture (the layout of the QNN and its trainable parameters), $\mathrm{C}$ and $\mathrm{W}$ also depend on the execution and training protocols (which include, e.g., the input data encoding and learning method). Applies universally, regardless of whether the learning machine and / or training involves quantum, classical, or hybrid operations, whether the trained parameters are classical or quantum, how many uses of the QNN (or repeats of the input data) occur per input, or how the data is encoded. 


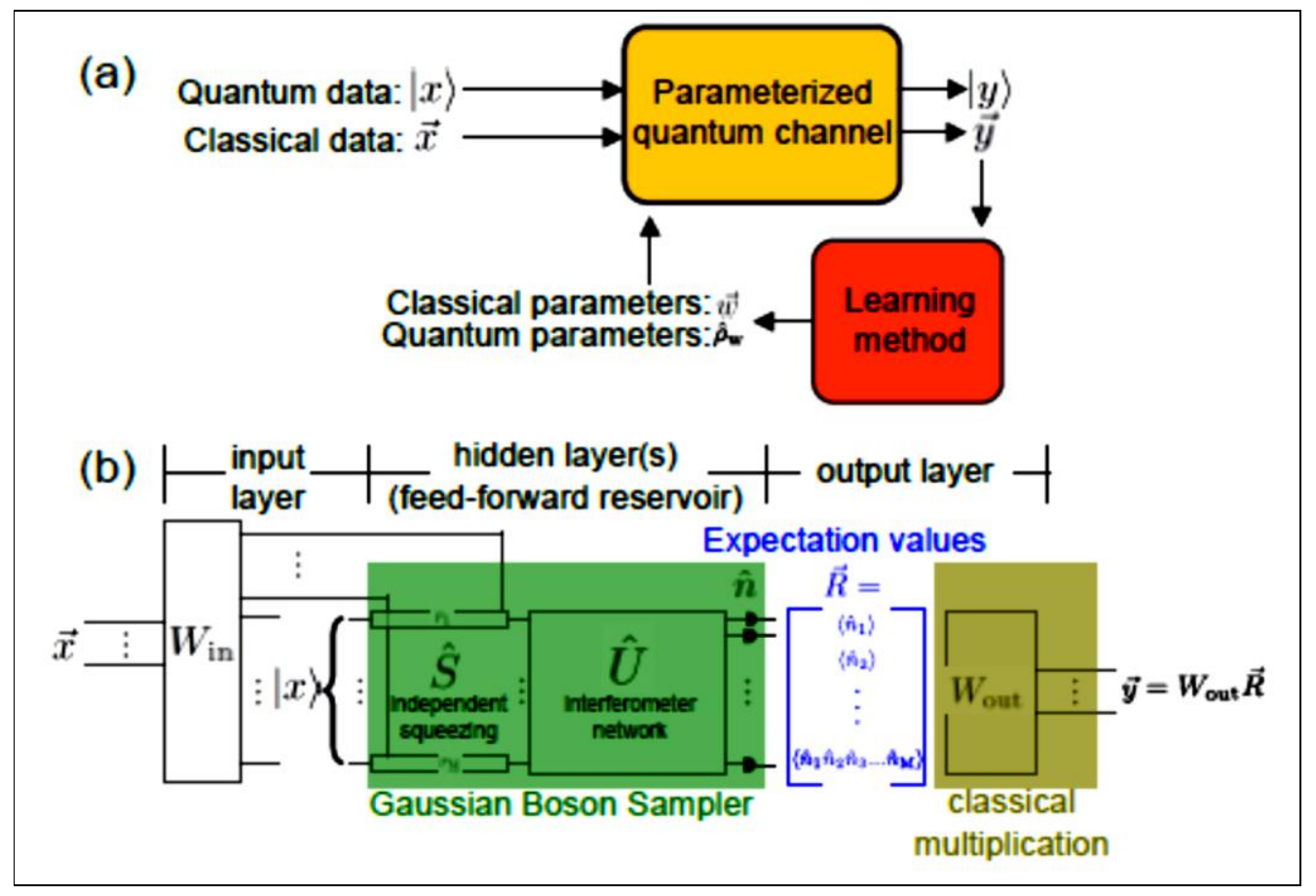

Figure 51. Schema of a general feed-forward QNN

Fig. 51a shows schema of a general feed-forward QNN, a parameterized quantum channel (which could include unitary and/or dissipative quantum evolutions, classical data processing, ancillary parameter states, etc.) which is trained in a supervised fashion to optimize the classical and quantum parameters $\vec{\omega}$ and / or $\rho_{\omega}$ so that the QNN best approximates the transformation implied by the training data. (b) Schematic of a feedforward quantum reservoir computer based on a Gaussian Boson Sampler. For classical tasks considered here, $|x\rangle=|0\rangle$ and data is then encoded through the squeezing parameters, and for all tasks we take $W_{\text {in }}$ to be the identify matrix [44].

Quantum tasks, such as preparing states or learning a quantum circuit, are unitary approximation tasks.

\subsection{EEG Data processing based on QNN}

EEG signals can be considered a realization of a random or stochastic process [8]. When an accurate description of the system is unavailable, a stochastic filter can be designed on the basis of probabilistic measures. Every solution to a stochastic filtering problem involves the computation of a time-varying probability density function $(p d f)$ on the state-space of the observed system. The architecture of recurrent quantum neural network RQNN model is based on the principles of QM with the Schrodinger wave equation (SWE) playing a major part. This approach enables the online estimation of a time-varying $p d f$ that allows estimating and removing the noise from the raw EEG signal.

Fig. 52a shows a basic architecture of RQNN model in which each neuron mediates a spatio-temporal field with a unified quantum activation function in the form of Gaussian that aggregates $p d f$ information from the observed noisy input signal. Thus, the solution of SWE (which is complex valued and whose modulus square is the $p d f$ that localizes the position of quantum object in the vector space) gives the activation function. From a mathematical point of view, the time-dependent single-dimension nonlinear SWE is a partial differential equation describing the dynamics of wave packet (modulus-square of this wave is the $p d f$ ) in the presence of a potential field (or function) (which is the force field in which the particles defined by the wave function are forced to move). Thus, the RQNN model is based on novel concept that a quantum object mediates the collective response of a neural lattice (a spatial structure of an array of neurons where each neuron is a simple computational unit as shown in Fig. 52a. 

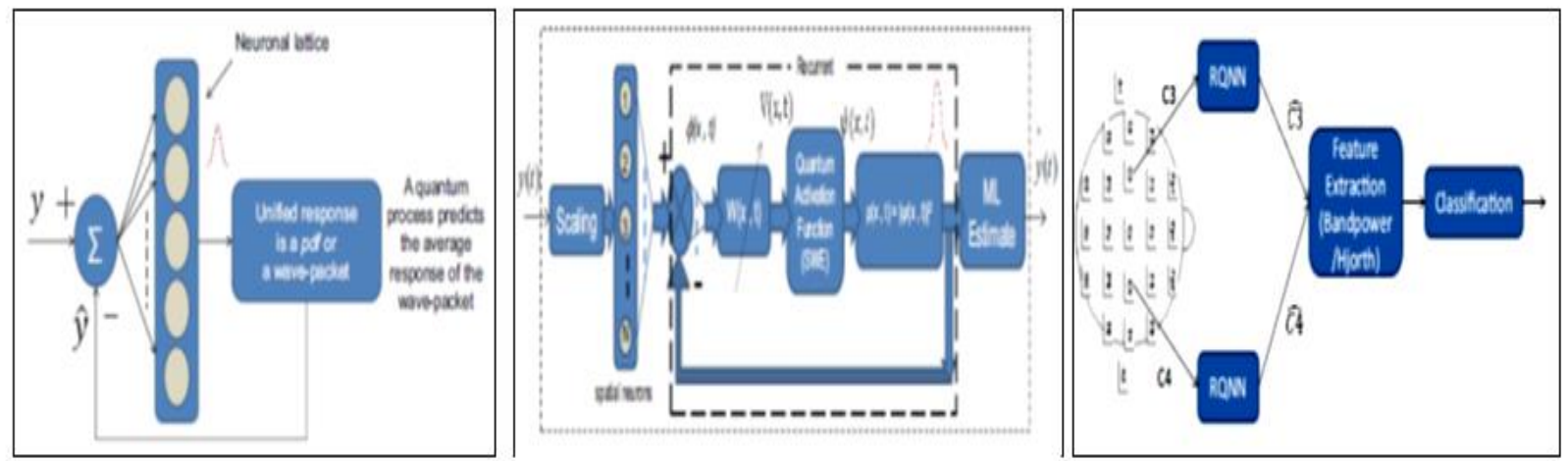

Figure 52. Conceptual framework of RQNN model (a) Signal estimation using RQNN model (b) RQNN model framework for EEG signal enhancement (c)

Such RQNN filter used for stochastic filtering is able to reduce noise, because of its stability being highly sensitive to model parameters, in case of imperfect tuning, the system may fail to track the signal and its output may saturate to absurd values. In the architecture used in Fig. 52b), the spatial neurons are excited by the input signal $y(t)$. The difference between the output of spatial neuronal network and the $p d f$ feedback $|\psi(x, t)|^{2}$ is weighted by a weight vector $W(x)$ to get the potential energy $V(x)$. The model can thus be seen as a Gaussian mixture model estimator of potential energy with fixed centers and variances, and only the weights are variable. These weights can be trained using any learning rule.

In the RQNN architecture (see Fig. 52b) makes the assumption that the average behavior of neural lattice that estimates the signal is a time-varying $p d f$ which is mediated by a quantum object placed in the potential field $V(x)$ and modulated by the input signal so as to transfer he information about $p d f$. SWE to recurrently track this $p d f$ because it is a well-known fact that the square of the modulus of $\psi$ function, the solution of the wave equation, is also a $p d f$.

The potential energy is calculated as $V(x)=\varsigma W(x, t) \phi(x, t)$, where

$$
\phi(x, t)=\exp \left(-\frac{(y(t)-x)^{2}}{2 \sigma^{2}}\right)-|\psi(x, t)|^{2}
$$

and $y(t)$ is the input signal and the synapses are represented by the time-varying synaptic weights $W(x, t)$. The variable $\zeta$ represents the scaling factor to actuate the spatial potential energy $V(x, t)$, and $\sigma$ is the width of the neurons in the lattice (taken here as unity). This potential energy modulates the nonlinear SWE described by:

$$
i \hbar \frac{\partial \psi(x, t)}{\partial t}=-\frac{\hbar^{2}}{2 m} \nabla^{2} \psi(x, t)+V(x, t) \psi(x, t)
$$

where $\psi(x, t)$ represents the quantum state, $\nabla$ is the Laplacian operator and $V(x, t)$ is the potential energy.

The neuronal lattice sets up the spatial potential energy $V(x)$. A quantum process described by the quantum state $\psi$ which mediates the collective response of neuronal lattice, evolves in this spatial potential $V$ $(x)$ according to (2). As $V(x)$ sets up the evolution path of the wave function, any desired response can be obtained by properly modulating the potential energy. Such RQNN filter used for stochastic filtering. Although this filter is able to reduce noise, because of its stability being highly sensitive to model parameters, in case of imperfect tuning, the system may fail to track the signal and its output may saturate to absurd values.

In the architecture used in this paper (Fig. 52b), the spatial neurons are excited by the input signal $y(t)$. The difference between the output of spatial neuronal network and the $p d f$. The filtered estimate is calculated using MLE as $\hat{y}(t)=E\left[|\psi(x, t)|^{2}\right]=\int x|\psi(x, t)|^{2} d x$, where $x$ represents the different possible values 
which may be taken up by the random process $y$. The variable $x$ can be interpreted as the discrete version of quantum space with the resolution within this discrete space being referred to as $\delta x$ (taken as 0.1 ). Thus, all the possible values of $x$ will construct the number of spatial neurons $N$ for RQNN model.

On the basis of MLE, the weights are updated and a new potential $V(x, t)$ is established for the next time evolution. It is expected that the synaptic weights $W(x, t)$ evolve in such a manner so as to drive the $\psi$ function to carry the exact information of $p d f$ of the filtered signal $\hat{y}(t)$. To achieve this goal, the weights are updated using the following learning rule:

$$
\frac{\partial W(x, t)}{\partial t}=-\beta_{d} W(x, t)+\beta \phi(x, t)\left(1+\vartheta(t)^{2}\right),
$$

where $\beta$ is the learning rate, and $\beta d$ is the delearning rate. Delearning is used to forget the previous information, as the input signal is not stationary, rather quasistationary in nature.

The second right-hand side term in the above equation maybe purely positive and so in the absence of delearning term, the value of synaptic weights $W$ may keep growing indefinitely. Delearning thus prevents unbounded increase in the values of the synaptic weights $W$ and does not let the system become unstable. The variable $v(t)$ in the second term is the difference between the noisy input signal and the estimated filtered signal, thereby representing the embedded noise as $\vartheta(t)=y(t)-\hat{y}(t)$. If the statistical mean of the noise is zero, then this error correcting signal $v(t)$ has less impact on weights, and it is the actual signal content in input $y(t)$ that influences the movement of wave packet along the desired direction which results in helping the goal of achieving signal filtering.

Figure 52c shows the position of RQNN model within the BCI system. The raw EEG signal is fed one sample at a time and an enhanced signal is obtained as a result of filtering process. The raw EEG is first scaled in the range $0-2$ before it is fed to the RQNN model. During the off-line classifier training process, all the trials from a particular channel of EEG are available. Therefore, the complete EEG is scaled using the maximum of amplitude value from that specific channel. During the online process, the EEG signal is approximately scaled in the range 0-2 using the maximum of amplitude value obtained from the off-line training data of that specific channel. The net effect is that the input signal during the online process is also maintained approximately in the region 0-2, and this enables the tracking of sample using a reduced range of the movement of wave packet. In addition, the number of spatial neurons has also been reduced along the $x$ axis from an earlier value of 401 to 612 in the present case. The primary assumption in doing this is that the unknown nonstationary and evolving EEG signal during the evaluation stage will stay within the bound of the range of 61 spatial neurons which can cover the input signal range up to three. If the scaling of the input signal is not implemented, then the number of neurons required to cover the input signal range will be larger thereby leading to an increased computational expense $[45,46]$.

\section{Conclusion}

One of the important tasks is the intelligent robust control systems is a control in unforeseen / unsharp situations. Modern solutions to this problem already make it possible to achieve good results, but such systems cannot be trained in on line, so the set of reaction methods to events is extremely limited. With the quantum computing and, in particular, the quantum fuzzy algorithm, it is possible to solve such problems by increasing the speed of deep machine learning. The use of quantum fuzzy inference can increase robustness without the expense of a time. One of the most optimal solutions in the design of intelligent robust control systems is the formation of knowledge bases for a variety of fixed control situations. The goal of a quantum regulator is to combine the knowledge bases obtained using the SCO into self-organizing quantum fuzzy regulators. Quantum deep machine learning on quantum artificial network and optimization on quantum genetic algorithm and applied examples in cognitive intelligent robotics in Part II considered. 


\section{References}

1. Petrov, B, Ulanov, G., Ulyanov S. and Hazen E. Information semantic problems in organization control. - M.: Nauka, 1977. - P. 452.

2. Ozer, E. and Feng, M. Structural reliability estimation with participatory sensing and mobile cyber-physical structural health monitoring systems // Appl. Sci. — 2019. - Pp. 2840.

3. Noor A. Potential of cognitive computing and cognitive systems // Open Eng. — 2015. - Vol. 5. — Pp. 75-88.

4. Chie H., Takato H. and Takayuki N. Deep Emotion: A Computational Model of Emotion Using Deep Neural Networks. CoRR, 2018, available at: http://arxiv.org/abs/1808.08447.

5. Rozaliev V. Postroenie matematicheskoj modeli emocij: Integrirovannye modeli i myagkie vychisleniya $\mathrm{v}$ iskusstvennom intellekte. // V Mezhdunarodnaya nauchno-prakticheskaya konferenciya // Integrirovannye modeli i myagkie vychisleniya v iskusstvennom intellekte" Sbornik nauchnyh trudov. -2009. - Pp. 950-957.

6. Bazgir O., Mohammadi Z. and Habibi S. Emotion recognition with machine learning using EEG signals // 25th National and 3rd International Iranian Conference on Biomedical Engineering (ICBME). - 2018. - Pp. 1-5.

7. Xie H., Pan J. and Wen Z. An EEG-based brain computer interface for emotion recognition and its application in patients with disorder of consciousness // IEEE Transactions on Affective Computing. - 2019. - Pp. 1-14.

8. Ulyanov S. and Litvintseva L. Intelligent robust control: soft computing technology. - M.: VNIIgeosistem, 2011. - P. 406.

9. Ulyanov S.V. (inventor) Self-organizing quantum robust control methods and systems for situations with uncertainty and risk // Patent US 8788450 B2. — 2014.

10. Sandberg H. Maximum work extraction and implementation costs for nonequilibrium Maxwell's demon // Physical Review E. — 2014. - No 4. - Pp. 042119.

11. Ulyanov S., Yamafuji K., Gradetsky V. and Fukuda T. Development of intelligent mobile robots for service use and mobile automation systems including wall climbing robots: Pt. 1. Fundamental design principles and motion models // International Journal of Intelligent Mechatronics: Design and Production. 1997. - Pp. 111-143.

12. Sagawa T, Ueda M. Minimal Energy Cost for Thermodynamic Information Processing: Measurement and Information Erasure // Phys. Rev. Lett. — 2012. - Vol. 102. — No 25. — Pp. 250602.

13. Horowitz J. M., Sandberg H. Second-law-like inequalities with information and their interpretations // New Journal of Physics. — 2014. — Vol. 16. — Pp. 125007.

14. Ulyanov S.V., Litvintseva L.V., Kurawaki I. et al. Principle of minimum entropy production in applied soft computing for advanced intelligent robotics and mechatronics // Soft Computing. — 2000. — Vol. 4. — No 3. - Pp. 141-146.

15. Sieniutycz S. Framework for optimal control in multistage energy systems // Physics Reports. - 2000. Vol. 326. - No 2.

16. Ulyanov S.V. Quantum relativistic informatics. LAP LAMBERT Academic Publishing, OmniScriptum GmbH \& Co. KG. - 2015.

17. Sadeghieh A., Roshanian J., Najafari F. Implementation of an intelligent adaptive controller for an electrohydravlic servo system based on a brain mechanism of emotional learning // Intern. J. of Advanced Robotic Systems (INTECH). - 2012. - Vol. 9. - Pp. 1-12.

18. Daryabeigi E., Zarchi A., Arab G.R., Markadeh M.A. Implementation of Emotional Controller (BELBIC) for Synchronous Reluctance Motor Drive Proc // IEEE Intern. Electric Machines \& Drivers Conf. (IEMDC). — 2011. — Pp. 1066-1093. 
19. Litvintseva L., Ulyanov I., Ulyanov S. Quantum fuzzy inference for knowledge base design in robust intelligent controllers // J. of Computer and Systems Sciences Intern. — 2007. — Vol. 46. — No 9. Pp. 908-961.

20. Ulyanov, S. V. Intelligent Robust Control System Based on Quantum KB-Self-organization: Quantum Soft Computing and Kansei / Affective Engineering Technologies // Springer International Publishing. - 2014. - Pp. 37-48.

21. Tanaka T., Ohii J., Litvintseva L., Yamafuji K., Ulyanov S. Intelligent control of a mobile robot for service use in office buildings and its soft computing algorithms // Journal of Robotics and Mechatronics. 1996. - Vol. 8. - Pp. 538-554.

22. Dawson G. and Toth K. Autism spectrum disorders. In D. Cicchetti \& D. J. Cohen (Eds.). // Developmental psychopathology: Risk, disorder, and adaptation. — 2006. — Pp. 317-357.

23. Stanton C., Kahn P. Jr., Severson R., Ruckert J. and Gill B. Robotic Animals Might Aid in the Social Development of Children with Autism // 3rd ACM/IEEE International Conference on Human-Robot Interaction, 2008.

24. Wei C., Wenxu S., Xinge L., Sixiao Zh., Ge Zh., Yanting W., Sailing H., Huilin Zh. and Jiajia Ch. Could Interaction with Social Robots Facilitate Joint Attention of Children with Autism Spectrum Disorder? // Computers in Human Behavior. - 2019. - Pp. 98.

25. Palestra, G., Carolis, B.D., Esposito, F. Artificial Intelligence for Robot-Assisted Treatment of Autism // WAIAH@AI*IA, 2017.

26. Cho, S.J. and Ahn, D. Socially Assistive Robotics in Autism Spectrum Disorder // Hanyang Medical Reviews. - 2016. - Vol. 36. - Pp. 17.

27. Rudovic O., Lee J., DaiM., Schuller B. and Picard R. W. Personalized Machine Learning for Robot Perception of Affect and Engagement in Autism Therapy // Science. - 2018. - Vol. 3.

28. Ulyanov S., Mamaeva A. and Shevchenko A. Programmnaya realizaciya modulya obrabotki dannyh dlya kognitivno-intellektual'noj sistemy dlya detej-autistov // «Sbornik dokladov XXV Mezhdunarodnoj konferencii «MATEMATIKA. KOMPYUTER. OBRAZOVANIE». — 2018. — Vol. 25. — Pp. 398.

29. Ulyanov S., Mamaeva A. and Shevchenko A. Kognitivno-intellektual'naya sistema diagnostiki, obucheniya i adaptacii detej-autistov. Chast. 1 // Sistemnyj analiz v nauke i obrazovanii. — 2016. — No 4. - URL: available at: http:/www.sanse.ru/archive/42.

30. Ulyanov S., Mamaeva A. and Shevchenko A. Kognitivno-intellektual'naya sistema diagnostiki, obucheniya i adaptacii detej-autistov. Chast 2. Opredelenie emocij // Programmnye produkty i sistemy: elektron. nauch. zhurnal. - 2017. - No 4. - URL: http://swsys-web.ru/cognitive-intellectual-systemfor-diagnosis-and-education-of-autistic- children-2.html.

31. Nikolaev A. Spektral'nye harakteristiki EEG na pervom etape resheniya razlichnyh prostranstvennyh zadach // Psihologicheskij zhurnal. 1994. — Vol. 15. — No 6. — Pp. 100-106.

32. Lapshina T. Psihofiziologicheskaya diagnostika emocij cheloveka po pokazatelyam EEG // Materialy Mezhdunarodnoj nauchno-prakticheskoj konferencii "Razvitie nauchnogo naslediya Borisa Mihajlovicha Teplova v otechestvennoj i mirovoj nauke" Nauchnyj sbornik. — M.: BF "Tverdislov", 2006. — Pp. 160165.

33. Fretska E., Bauer H., Leodolter M. and Leodolter U. Loss of control and negative emotions: a cortical slow potential topography study // International Journal of Psychophysiology. — 1999. - Vol. 33. Pp. 127-141.

34. Ulyanov S., Reshetnikov A. and Mamaeva A. Gibridnye kognitivnye nechetkie sistemy upravleniya avtonomnym robotom na osnove nejrointerfejsa i tekhnologii myagkih vychislenij // Programmnye produkty i sistemy / Software \& Systems. - 2017. - Vol. 30. — No 3. - Pp. 420-424.

35. Ulyanov S.V., Yamafuji K. Fuzzy intelligent emotion and instinct control of a robotic unicycle // In Proc. 4th Intern. Workshop on Advanced Motion Control. — 1996. — Japan, Mie. — Vol. 1. — Pp. 127-132. 
36. Ulyanov S.V., Watanabe S., Yamafuji K. A new physical measure for mechanical controllability of a robotic unicycle on basis of intuition, instinct and emotion computing // In Proc. 2nd Intern. Conf. on Application of Fuzzy Systems and Soft Computing. - 1996. - Pp. 78-92.

37. Ulyanov V.S., Ohkura T., Yamafuji K., Ulyanov S.V. Intelligent control of an extension-less robotic unicycle: A study of mechanical controllability via minimum entropy criteria // Lecture Notes in Control and Information Sciences: Progress in System and Robot Analysis and Control Design. — 1999. — Vol. 243. - Pp. 559-570.

38. Hagiwara T., Ulyanov S.V., Takahashi K., Diamante O. An ap-plication of a smart control suspension system for a passenger car based on soft computing // Yamaha Motor Technical Report. — 2003.01.15.

39. EU PCT Patent WO 2004/012139 A2 (PCT/US2003/023727), "Intelligent mechatronic control suspension system based on quantum soft computing" (Inventor: S. V. Ulyanov). - International publication Date: 5 February 2004 (US Patent US 2004/0024750 A1. Publ. Date: Feb. 5, 2004).

40. US Patent No 2006,0218 A1, "System for soft computing simulation" (Inventor: S. V. Ulyanov). — Date of patent: Sept. 2006.

41. Ulyanov S. Intelligent self-organized robust control design based on quantum / soft computing technologies and Kansei engineering // Computer Science Journal of Moldova. — 2013. — Vol. 21. — N0 62. Pp. 242-279.

42. Ulyanov S.V., Yamafuji K. Intelligent self-organized cognitive controllers. Pt. 1: Kansei / affective engineering and quantum / soft computing technologies // System Analysis in Science and Education. - 2014. — No 4. URL: http:/www.sanse.ru/archive/48.

43. Kak S. On Quantum Neural Computing // Inf. Sci. — 1995. — Vol. 83. - Pp 143-160.

44. Menner T. Quantum Artificial Neural Networks. — Univ. of Exeter, UK. — 1998.

45. Gandhi V., Prasad G., Coyle D. et all. Quantum neural network-based EEG filtering for a brain-computer interface // IEEE Trans. on Neural Network and Learning Systems. 2014. - Vol. 25. — No 2. - Pp. 278-288.

46. Ulyanov S.V., Feng M., Ulyanov V.S., Yamafuji K., Fukuda T., Arai F. Stochastic analysis of timevariant nonlinear dynamic systems. Part 1: the Fokker-Planck-Kolmogorov equation approach in stochastic mechanics // Prob. Engng. Mech. - 1998. - Vol. 13. - No 3. - Pp. 183-203.

47. Elio Conte, Rui Freire Lucas. First Time Demonstration of the Quantum Interference Effect during Integration of Cognition and Emotion in Children // World Journal of Neuroscience. - 2015. - No 5. Pp. 91-98.

48. Aerts D., Sozzo A. Quantum Interference in Cognition: Structural Aspects of the Brain // arXiv:1204.4914v1 [cs.AI] 22 Apr 2012.

49. Clark K. Basis for a neuronal version of Grover's quantum algorithm // Frontiers in Molecular Neuroscience. - 2014. — Vol. 4. - No. 3. - Pp. 325-329. — doi: 10.3389/fnmol.2014.0002 http://community.frontiersin.org/people/u/52068.

50. Alexander J. Shackman, Tim V. Salomons, Heleen A. Slagter, Andrew S. Fox, Jameel J. Winter and Richard J. Davidson. The integration of negative affect, pain and cognitive control in the cingulate cortex // Nature. - 2011. — Vol. 12. 